\title{
FORMATION AND EVOLUTION OF PLANETARY SYSTEMS: PROPERTIES OF DEBRIS DUST AROUND SOLAR-TYPE STARS
}

\author{
John M. Carpenter ${ }^{1}$, Jeroen Boumman ${ }^{2}$, Eric E. Mamajek ${ }^{3}$, Michael R. Meyer ${ }^{4}$, Lynne A. Hillenbrand ${ }^{1}$, Dana \\ E. Backman ${ }^{5}$, Thomas Henning ${ }^{2}$, Dean C. Hines ${ }^{6}$, David Hollenbach ${ }^{7}$, Jinyoung Serena Kim $^{4}$, Amaya Moro-Martin ${ }^{8}$, \\ Ilaria Pascucci $^{4}$, Murray D. Silverstone ${ }^{9}$, John R. Stauffer ${ }^{10}$, and Sebastian Wolf ${ }^{2}$ \\ ${ }^{1}$ Department of Astronomy, California Institute of Technology, Mail Code 105-24, 1200 East California Boulevard, Pasadena, CA 91125, USA \\ ${ }^{2}$ Max-Planck-Institut für Astronomie, D-69117 Heidelberg, Germany \\ ${ }^{3}$ Harvard-Smithsonian Center for Astrophysics; Current address: Department of Physics \& Astronomy, University of Rochester, Rochester, NY 14627, USA \\ ${ }^{4}$ Steward Observatory, The University of Arizona, 933 North Cherry Avenue, Tucson, AZ 85721, USA \\ ${ }^{5}$ SOFIA/SETI Institute \\ ${ }^{6}$ Space Science Institute, 4750 Walnut Street, Suite 205, Boulder, CO 80301, USA \\ ${ }^{7}$ NASA-Ames Research Center \\ ${ }^{8}$ Department of Astrophysical Science, Princeton University, USA \\ ${ }^{9}$ Eureka Scientific, Inc., 113 Castlefern Dr., Cary, NC 25713, USA \\ ${ }^{10}$ Spitzer Science Center, California Institute of Technology, Mail Code 314-6, 1200 East California Boulevard, Pasadena, CA 91125, USA \\ Received 2008 June 22; accepted 2008 September 30; published 2009 March 5
}

\begin{abstract}
We present Spitzer photometric (IRAC and MIPS) and spectroscopic (IRS low resolution) observations for 314 stars in the Formation and Evolution of Planetary Systems Legacy program. These data are used to investigate the properties and evolution of circumstellar dust around solar-type stars spanning ages from approximately $3 \mathrm{Myr}-$ 3 Gyr. We identify 46 sources that exhibit excess infrared emission above the stellar photosphere at $24 \mu \mathrm{m}$, and 21 sources with excesses at $70 \mu \mathrm{m}$. Five sources with an infrared excess have characteristics of optically thick primordial disks, while the remaining sources have properties akin to debris systems. The fraction of systems exhibiting a $24 \mu \mathrm{m}$ excess greater than $10.2 \%$ above the photosphere is $15 \%$ for ages $<300 \mathrm{Myr}$ and declines to $2.7 \%$ for older ages. The upper envelope to the $70 \mu \mathrm{m}$ fractional luminosity appears to decline over a similar age range. The characteristic temperature of the debris inferred from the IRS spectra range between 60 and $180 \mathrm{~K}$, with evidence for the presence of cooler dust to account for the strength of the $70 \mu \mathrm{m}$ excess emission. No strong correlation is found between dust temperature and stellar age. Comparison of the observational data with disk models containing a power-law distribution of silicate grains suggests that the typical inner-disk radius is $\gtrsim 10 \mathrm{AU}$. Although the interpretation is not unique, the lack of excess emission shortward of $16 \mu \mathrm{m}$ and the relatively flat distribution of the $24 \mu \mathrm{m}$ excess for ages $\lesssim 300 \mathrm{Myr}$ is consistent with steady-state collisional models.
\end{abstract}

Key words: circumstellar matter - infrared: stars - planetary systems: formation

\section{INTRODUCTION}

The IRAS mission left a lasting legacy with the surprise discovery of dust surrounding Vega and other main-sequence stars (Aumann et al. 1984; Aumann 1985). The orbital lifetime of the dust, limited by collisional and radiative processes in a gas poor environment, is orders of magnitude shorter than the stellar age, and implies that the dust must have been created recently (Backman \& Paresce 1993). The model currently favored to explain the presence of dust in these systems postulates that planets gravitationally stir a population of planetesimals, which subsequently collide and fragment into a cascade of smaller debris (Williams \& Wetherill 1994). In support of this conjecture, high-angular resolution images have indeed shown that the dust is frequently distributed in disklike or ringlike geometries (Smith \& Terrile 1984; Koerner et al. 1998; Holland et al. 1998; Kalas et al. 2004; Schneider et al. 2006).

The presumed causal relationship between debris dust and planetary systems has motivated many studies to investigate the frequency and properties of debris disks (Aumann \& Probst 1991; Mannings \& Barlow 1998; Moór et al. 2006; Rhee et al. 2007, see also Lagrange et al. 2000 and references therein). Analysis of IRAS data indicates that $\sim 15 \%$ of nearby AFGK-type stars are surrounded by a debris disk with a dust-to-photosphere luminosity ratio exceeding that of the Vega debris disk (Backman \& Gillett 1987; Plets \& Vynckier
1999). Subsequent observations with $I S O$ demonstrated that the frequency of debris disks declines with age (Habing et al. 1999, 2001; Spangler et al. 2001). Decin et al. (2003) emphasized, however, that a large range of dust properties are present at any given stellar age, which suggests that planetesimals with a wide range of properties exist around main-sequence stars.

As an extension of the IRAS and ISO heritage, the high photometric precision and extraordinary sensitivity of the Spitzer Space Telescope (Werner et al. 2004) have enabled a comprehensive characterization on how the debris disk phenomenon correlates with stellar mass and age. Beichman et al. (2005b) obtained the first secure demonstration of debris dust around extrasolar planetary systems (see also Moro-Martín et al. 2007a) to support the basic tenet that debris and planets are connected. Beichman et al. (2006b) showed that fractional luminosity from dust in the $1 \mathrm{AU}$ region around solar-type stars is rare at levels more than 1400 times the brightness of the current zodiacal cloud, although spectacular examples of warm $(\sim 300 \mathrm{~K})$ dust have been discovered (Song et al. 2005; Beichman et al. 2005a; Rhee et al. 2008). Rieke et al. (2005) found that $\sim 47 \%$ of A-type stars younger than $90 \mathrm{Myr}$ have a $24 \mu \mathrm{m}$ excess more than $25 \%$ above the photosphere, with a rapid decline in both the frequency and magnitude of the excess toward older ages. Lower mass FGK-type stars exhibit a similar decline in the warm excess fraction with age (Siegler et al. 2007), although the persistence time may be longer for $70 \mu \mathrm{m}$ excess emission 
(Su et al. 2006; Bryden et al. 2006). For stars older than 600 Myr, the presence of $70 \mu \mathrm{m}$ excess shows no clear dependence on the spectral type for AFGK stars (Trilling et al. 2008). Few debris disks have been detected around M dwarfs with Spitzer (Beichman et al. 2006a; Gautier et al. 2007); it remains uncertain however whether this indicates a real difference in disk frequency compared to higher-mass stars, or simply reflects the greater difficulty in detecting dust around low-luminosity stars (see, e.g., Cieza et al. 2008).

The improved observational data have coincided with increasingly sophisticated models to understand the connection between debris dust and the presence of planets. Liou \& Zook (1999) demonstrated how debris dust can be trapped in resonances with orbiting planets to produce the asymmetric and clumpy structure observed in some debris disks (see also Wyatt 2003; Moro-Martín \& Malhotra 2003, 2005; Krivov et al. 2007). In a series of papers, Kenyon \& Bromley (2001, 2002, 2004, 2005) investigated the link between the collisional growth of planets (see Safronov 1969; Greenberg et al. 1984; Wetherill \& Stewart 1989; Spaute et al. 1991) and the subsequent production of debris in the collisional cascade. They demonstrated that as planet formation proceeds from the inner disk outward and gravitationally stirs the planetesimals, debris dust will be produced at sufficient levels to be detected with current instrumentation. Dominik \& Decin (2003) suggest that the rapid decline in the observed debris emission at an age of a few hundred million years is a consequence of the collisional depletion of the planetesimal belts. Wyatt et al. (2007a) extended their model and confirmed the basic findings, and also identified several luminous debris systems which are best explained by a recent collision between planetesimals that produced copious amounts of dust.

To date, the observational data and the application of models are most complete for A stars which are sufficiently bright to be detected to large distances by IRAS and ISO. With the sensitivity of Spitzer, extensive data sets can now be collected for solar-type stars that may provide insights on the evolutionary history of our own solar system. Toward this goal, the Formation and Evolution of Planetary Systems (FEPS) Spitzer Legacy Program (Meyer et al. 2006) has conducted a photometric and spectroscopic survey of 328 solar-type stars spanning ages between $\sim 3 \mathrm{Myr}$ and 3 Gyr. Previous FEPS studies have analyzed subsets of the FEPS data to address specific issues regarding debris evolution, including (1) the evolution of terrestrial temperature debris around stars younger than $30 \mathrm{Myr}$ (Silverstone et al. 2006), (2) the temporal evolution of warm $24 \mu \mathrm{m}$ excess (Stauffer et al. 2005; Meyer et al. 2008), (3) the identification and properties of cool Kuiper-Belt analogs (Meyer et al. 2004; Kim et al. 2005; Hillenbrand et al. 2008), (4) analysis of debris disk properties around known extra-solar planetary systems (MoroMartín et al. 2007a, 2007b), (5) dust composition for a sample of optically thick accretion disks (Bouwman et al. 2008), and (6) the gas dissipation timescales in debris disks (Hollenbach et al. 2005; Pascucci et al. 2006, 2007). In this contribution, we present the most comprehensive analysis to date of photometry and low-resolution spectra in the FEPS survey to investigate the evolution of debris disks around solar-type stars. The broad goal of our investigation is to quantify the incidence of the debris phenomenon around solar-analogs, infer the properties of the implied planetesimal belts around these systems, and understand the similarities and differences of these presumed planetary systems with the solar system.

Carpenter et al. (2008, hereafter Paper I) describe the observation and data reduction procedures adopted for this study.
Table 1

Summary of Sample by Stellar Association

\begin{tabular}{|c|c|c|c|c|c|}
\hline \multirow[t]{2}{*}{ Group } & \multirow{2}{*}{$\begin{array}{c}\text { Age Range } \\
\text { (Myr) }\end{array}$} & \multirow[t]{2}{*}{ Number Stars } & \multicolumn{3}{|c|}{ Spectral Type } \\
\hline & & & $\mathrm{F}$ & $\mathrm{G}$ & K \\
\hline Corona Australis & $3-10$ & 7 & 0 & 2 & 5 \\
\hline Upper Scorpius & 5 & 16 & 0 & 8 & 8 \\
\hline Upper Centaurus Lupus & 15 & 23 & 1 & 13 & 9 \\
\hline Lower Centaurus Crux & 17 & 14 & 0 & 9 & 5 \\
\hline IC 2602 & 55 & 5 & 0 & 4 & 1 \\
\hline Alpha Per & 80 & 13 & 6 & 6 & 1 \\
\hline Pleiades & 120 & 20 & 5 & 14 & 1 \\
\hline Hyades & 600 & 22 & 5 & 10 & 7 \\
\hline Field & $3-3000$ & 194 & 22 & 131 & 41 \\
\hline
\end{tabular}

A summary of the FEPS sample and important aspects of the FEPS data reduction are summarized in Section 2. In Section 3, we analyze each of the Spitzer data products to identify stars that have an infrared excess between 3.6 and $70 \mu \mathrm{m}$. We use these results to trace the temporal evolution of debris properties (Section 4), and to constrain the location of the planetesimal belts (Section 5). We then compare these properties to the solar system debris disk (Section 6) before summarizing our results (Section 7).

\section{STELLAR SAMPLE AND OBSERVATIONS}

This paper analyzes data for 314 stars that were selected for the FEPS program without regard to the presence or absence of a known infrared excess from pre-Spitzer observations. We exclude 14 other FEPS stars with suspected excesses from $I R A S$ or ISO that were observed explicitly for a gas-detection experiment (Hollenbach et al. 2005; Pascucci et al. 2006, 2007). Meyer et al. (2006) describe the sample properties in detail and only a summary is presented here. The stars span spectral types between $\mathrm{K} 7$ and F5 and ages between approximately $3 \mathrm{Myr}$ and 3 Gyr. The stellar masses range from 0.7 to $2.2 M_{\odot}$, with $90 \%$ of the stars having masses less than $1.4 M_{\odot}$. Stellar ages were estimated by a variety of methods. In brief, for the youngest clusters/associations, the ages were based on fits to pre-mainsequence isochrones; for older clusters, the ages are primarily based on isochronal fits to the upper main-sequence turnoff and/or the "lithium-depletion boundary" method (Rebolo et al. 1992). Field stars were assigned ages based on stellar activity indicators (e.g., X-ray, rotation, Ca II H\&K), whose decline in strength with age has been calibrated with respect to open clusters of known age (Mamajek \& Hillenbrand 2008).

The sample breakdown between field stars and associations is provided in Table 1 and by stellar age in Table 2. The adopted ages for individual stars are listed in Table 3. Stellar ages are distributed approximately in uniform logarithmic intervals with between 34 and 60 stars for every factor of 3 in age. For ages less than $30 \mathrm{Myr}$, the sample is weighted toward stars found in associations, and stars older than 1 Gyr consist entirely of field stars because of the lack of nearby, old clusters. Younger stars tend to have later spectral types since solar-mass stars increase in temperature as they evolve from the pre-main-sequence phase to the main sequence.

FEPS obtained IRAC 3.6, 4.5, and $8 \mu \mathrm{m}$ images, ${ }^{11}$ MIPS 24 and $70 \mu \mathrm{m}$ images, and IRS low-resolution spectra between

\footnotetext{
11 The FEPS IRAC observations were obtained in subarray mode, where a subsection of the full array is read out to enable observations of bright stars. Subarray observations obtain images in the four IRAC band separately. IRAC $5.8 \mu \mathrm{m}$ observations were not obtained for the majority of the FEPS targets (see Paper I).
} 
Table 2

Summary of Sample by Stellar Age

\begin{tabular}{|c|c|c|c|c|c|c|c|c|}
\hline \multirow{2}{*}{$\begin{array}{l}\text { Age Range } \\
(\mathrm{Myr})\end{array}$} & \multicolumn{3}{|c|}{ Number } & \multicolumn{2}{|c|}{ Percentage } & \multicolumn{3}{|c|}{ Spectral Type } \\
\hline & Total & Associations & Field & Associations & Field & $\mathrm{F}$ & $\mathrm{G}$ & $\mathrm{K}$ \\
\hline$<10$ & 34 & 21 & 13 & 62 & 38 & 1 & 15 & 18 \\
\hline $10-30$ & 49 & 39 & 10 & 80 & 20 & 2 & 26 & 21 \\
\hline $30-100$ & 59 & 18 & 41 & 31 & 69 & 10 & 37 & 12 \\
\hline $100-300$ & 60 & 20 & 40 & 33 & 67 & 11 & 38 & 11 \\
\hline $300-1000$ & 55 & 22 & 33 & 40 & 60 & 6 & 38 & 11 \\
\hline$\geqslant 1000$ & 57 & 0 & 57 & 0 & 100 & 10 & 42 & 5 \\
\hline All & 314 & 120 & 194 & 38 & 62 & 40 & 196 & 78 \\
\hline
\end{tabular}
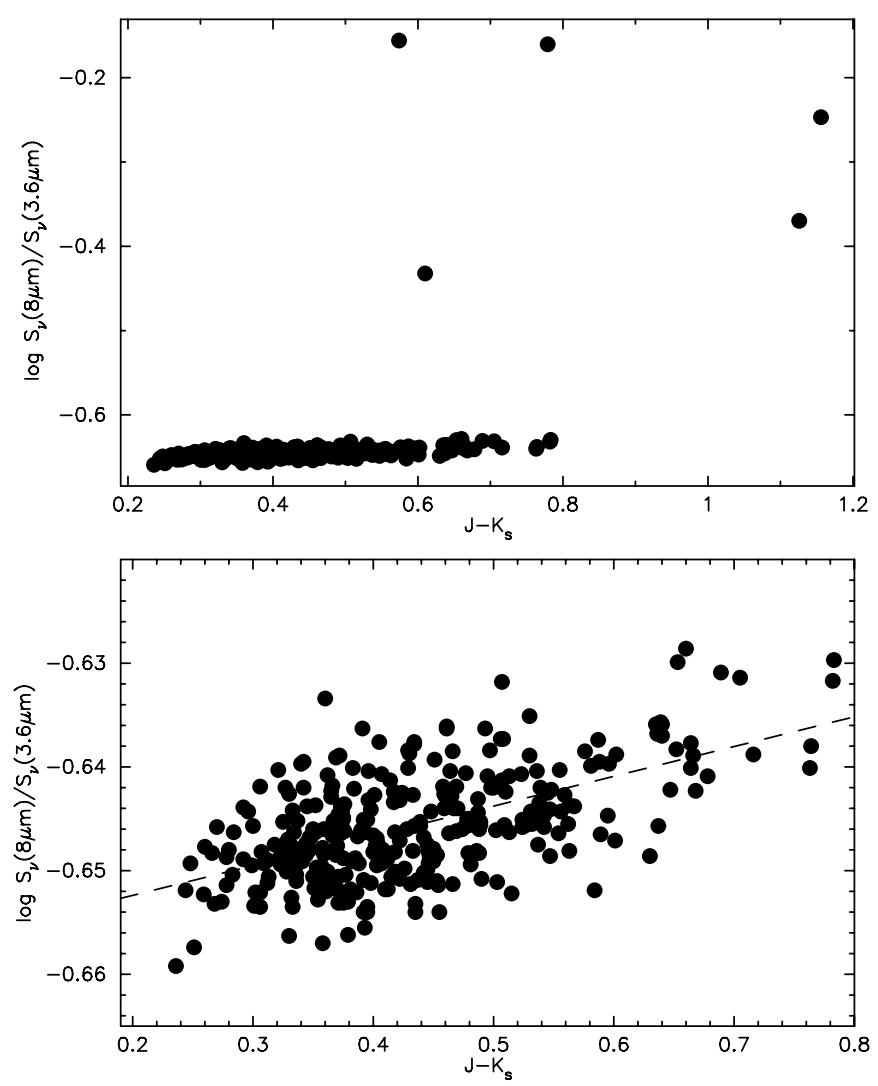

Figure 1. Plot of dereddened 2MASS $J-K_{\mathrm{S}}$ color vs. the $8 \mu \mathrm{m}$ to $3.6 \mu \mathrm{m}$ flux ratio $\left(R_{8 / 3.6}\right)$ for 309 stars in the FEPS program. Five FEPS stars were omitted that do not have high-quality (PHQUAL $=\mathrm{A}$; see Cutri et al. 2003) 2MASS $J$ or $K_{\mathrm{s}}$ photometry. The top panel shows data for all stars, and the bottom panel excludes five stars with flux ratio greater than 0.25 that have infrared excesses from optically thick disks (Silverstone et al. 2006). The dashed line in the bottom panel represents a linear fit to the data $\left(\log \left(R_{8 / 3.6}\right)=0.029\left(J-K_{\mathrm{S}}\right)-0.658\right)$.

8 and $35 \mu \mathrm{m}$ (SL1, LL1, and LL2 orders) for most of the sample, with observations for a few stars supplemented from the Spitzer archive. Five (HD 80606, HD 139813, H II 2881, HIP 42491, and RX J1544.0-3311) of the 314 sources do not have an IRS spectrum since the peak-up observations selected the incorrect star. In a few sources, portions of the IRS spectra appear corrupted and were discarded. The flux densities in the LL spectra for ScoPMS 52 are $\sim 60 \%$ higher than the MIPS $24 \mu \mathrm{m}$ flux density, and the IRS spectrum is likely contaminated by a source $18^{\prime \prime}$ away that has an order of magnitude higher flux density. The flux density in the SL1 spectrum for HD 13974 is $2.6 \times$ lower than expected, and the extracted LL1 and LL2 spectra for R45 have negative flux densities. Also, the IRS spectra for two sources (H II 1015 and HE 699) were discarded because of poor signal to noise. Finally, since Paper I was published, we have reanalyzed the spectrum for the star 1RXS J051111.1+281353 using the S15 data products from the Spitzer Science Center.

The signal-to-noise ratio $(\mathrm{S} / \mathrm{N})$ on the stellar photosphere is $\gtrsim 30$ for both the IRAC and MIPS $24 \mu \mathrm{m}$ photometry. At $70 \mu \mathrm{m}$, the photosphere was detected above the $3 \sigma$ noise level for only one source. The $\mathrm{S} / \mathrm{N}$ of the spectra was assessed by computing synthetic broad-band fluxes at $16 \mu \mathrm{m}$ using a squarewave response function between 15 and $17 \mu \mathrm{m}$, at $24 \mu \mathrm{m}$ using the MIPS $24 \mu \mathrm{m}$ instrumental response function, ${ }^{12}$ and at $32 \mu \mathrm{m}$ using a square-wave response function between 30 and $34 \mu \mathrm{m}$. In the $16 \mu \mathrm{m}$ bandpass, the $\mathrm{S} / \mathrm{N}$ of the IRS spectra is $\geqslant 20$ for 305 sources. At $24 \mu \mathrm{m}$, the $\mathrm{S} / \mathrm{N}$ is $\geqslant 10$ for 302 stars and $\geqslant 5$ in 305 sources. At $32 \mu \mathrm{m}$, the $\mathrm{S} / \mathrm{N}$ is $\geqslant 5$ for 264 sources and $\geqslant 3$ for 292 stars. A complete description of the data reduction procedures is presented in Paper I.

\section{IDENTIFYING SOURCES WITH INFRARED EXCESSES}

In this section, we analyze the photometric and spectroscopic data to identify sources that exhibit infrared emission diagnostic of circumstellar dust. Each Spitzer instrument is sensitive to dust emitting over a range of dust temperatures, and a given source will not necessarily exhibit detectable infrared excesses in all instruments. In general terms, IRAC photometry is most sensitive to hot dust located in the terrestrial planet zone (as defined by our solar system), MIPS $24 \mu \mathrm{m}$ to warm dust in the gas-giant formation region, and MIPS $70 \mu \mathrm{m}$ to cold Kuiper Belt analogs. IRS spectra probe regions similar to that by IRAC and MIPS $24 \mu \mathrm{m}$. We first analyze the data for each instrument individually, and then synthesize the results in Section 3.6 to identify a reliable sample of excess sources. In subsequent sections, we analyze these data to infer disk properties.

\subsection{IRAC}

In the top panel of Figure 1, we present the $8 \mu \mathrm{m}$ to $3.6 \mu \mathrm{m}$ flux ratio ( $\left.\equiv R_{8 / 3.6}\right)$ as a function of $J-K_{\mathrm{S}}$ color for the FEPS sample after dereddening the photometry using the extinction corrections derived in Paper I and the reddening law compiled by Mathis (1990). The median visual extinction for the sample is $0 \mathrm{mag}$ with a maximum of $1.8 \mathrm{mag}$. Sources with large values of $R_{8 / 3.6}$ contain a possible infrared excess at $8 \mu \mathrm{m}$. Since the youngest stars in our sample are $\sim 3 \mathrm{Myr}$ old, and the inner disk, as traced by photometric observations at $\lambda<3.5 \mu \mathrm{m}$, dissipates in half of solar-type stars by an age of $3 \mathrm{Myr}$ (Haisch et al. 2001), we anticipate that the $J-K_{\mathrm{s}}$ color traces the stellar

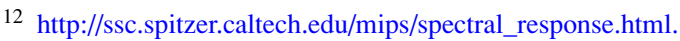


Table 3

Source List

\begin{tabular}{|c|c|c|c|c|c|c|c|c|c|c|c|}
\hline \multirow[t]{2}{*}{ Name } & \multirow{2}{*}{$\begin{array}{l}\text { Stellar } \\
\text { Group }^{\mathrm{a}}\end{array}$} & \multirow[t]{2}{*}{$\log$ (age/years) } & \multirow{2}{*}{$\begin{array}{c}\left(J-K_{\mathrm{S}}\right)_{\mathrm{o}}{ }^{\mathrm{b}} \\
(\mathrm{mag})\end{array}$} & \multicolumn{3}{|c|}{ Normalized Ratios $^{\mathrm{c}}$} & \multirow[t]{2}{*}{$\log p^{\mathrm{d}}$} & \multicolumn{4}{|c|}{ Excess? } \\
\hline & & & & $R_{16 / 8}$ & $R_{24 / 8}$ & $R_{70 / 8}$ & & $8 \mu \mathrm{m} 16 \mu \mathrm{m}$ & IRS & $24 \mu \mathrm{m}$ & $70 \mu \mathrm{m}$ \\
\hline 1E $0307.4+1424$ & Field & 7.8 & 0.40 & $0.92 \pm 0.05$ & $1.06 \pm 0.03$ & $-41.9 \pm 21.0$ & -0.73 & & & & \\
\hline 1E $0324.1-2012$ & Field & 7.8 & 0.37 & $1.16 \pm 0.05$ & $1.08 \pm 0.03$ & $-7.1 \pm 15.0$ & -2.52 & & & & \\
\hline 1RXS J025216.9+361658 & Field & 7.8 & 0.69 & $0.81 \pm 0.05$ & $0.94 \pm 0.03$ & $-0.6 \pm 4.3$ & -1.37 & & & & \\
\hline 1RXS J025751.8+115759 & Field & 7.8 & 0.49 & $0.95 \pm 0.05$ & $0.97 \pm 0.03$ & $3.6 \pm 15.0$ & -0.16 & & & & \\
\hline 1RXS J030759.1+302032 & Field & 8.2 & 0.43 & $0.95 \pm 0.05$ & $0.96 \pm 0.03$ & $2.2 \pm 5.4$ & -1.18 & & & & \\
\hline 1RXS J031644.0+192259 & Field & 7.7 & 0.43 & $1.09 \pm 0.05$ & $1.03 \pm 0.03$ & $-57.8 \pm 36.9$ & -0.68 & & & & \\
\hline 1RXS J031907.4+393418 & Field & 7.8 & 0.49 & $0.98 \pm 0.05$ & $1.02 \pm 0.03$ & $-22.1 \pm 31.6$ & -0.23 & & & & \\
\hline 1RXS J034423.3+281224 & Field & 7.8 & 0.38 & $1.01 \pm 0.05$ & $0.96 \pm 0.03$ & $-0.4 \pm 4.9$ & -1.44 & & & & \\
\hline 1RXS J035028.0+163121 & Field & 7.8 & 0.45 & $0.96 \pm 0.05$ & $1.00 \pm 0.03$ & $-32.3 \pm 21.1$ & -0.26 & & & & \\
\hline 1RXS J043243.2-152003 & Field & 6.6 & 0.43 & $0.95 \pm 0.05$ & $1.01 \pm 0.03$ & $-7.8 \pm 11.4$ & -1.82 & & & & \\
\hline 1RXS J051111.1+281353 & Field & 6.7 & 0.51 & $1.02 \pm 0.05$ & $1.23 \pm 0.03$ & $2.0 \pm 15.8$ & -0.94 & & $\mathrm{Y}$ & & \\
\hline 1RXS J053650.0+133756 & Field & 8.2 & 0.53 & $0.90 \pm 0.05$ & $0.98 \pm 0.03$ & $-0.3 \pm 20.6$ & -4.54 & & Y & & \\
\hline 2RE J0255+474 & Field & 7.9 & 0.68 & $1.00 \pm 0.05$ & $0.97 \pm 0.03$ & $-1.1 \pm 4.0$ & -0.27 & & & & \\
\hline AO Men & Field & 7.1 & 0.72 & $1.10 \pm 0.05$ & $1.02 \pm 0.02$ & $2.8 \pm 2.0$ & -0.27 & & & & \\
\hline AP 93 & $\alpha$ Per & 7.9 & 0.58 & $0.98 \pm 0.05$ & $1.05 \pm 0.03$ & $32.3 \pm 62.4$ & -0.46 & & & & \\
\hline B102 & IC 2602 & 7.7 & 0.43 & $0.94 \pm 0.05$ & $1.04 \pm 0.03$ & $-57.9 \pm 85.3$ & -25.38 & & $\mathrm{Y}$ & & \\
\hline BPM 87617 & Field & 8.1 & 0.78 & $0.99 \pm 0.05$ & $0.95 \pm 0.03$ & $-4.0 \pm 5.7$ & -0.32 & & & & \\
\hline HD 105 & Field & 7.5 & 0.35 & $0.99 \pm 0.05$ & $1.09 \pm 0.02$ & $50.1 \pm 5.2$ & -18.90 & & $\mathrm{Y}$ & $\mathrm{Y}$ & $\mathrm{Y}$ \\
\hline HD 377 & Field & 7.6 & 0.31 & $1.06 \pm 0.05$ & $1.38 \pm 0.02$ & $56.5 \pm 6.0$ & -45.36 & & $\mathrm{Y}$ & $\mathrm{Y}$ & $\mathrm{Y}$ \\
\hline HD 691 & Field & 8.5 & 0.40 & $0.99 \pm 0.05$ & $1.02 \pm 0.02$ & $3.1 \pm 2.7$ & -3.00 & & $\mathrm{Y}$ & & \\
\hline HD 984 & Field & 7.6 & 0.33 & $0.81 \pm 0.05$ & $1.01 \pm 0.02$ & $-3.2 \pm 2.9$ & -0.39 & & & & \\
\hline HD 6434 & Field & 9.5 & 0.39 & $1.03 \pm 0.05$ & $0.99 \pm 0.02$ & $3.0 \pm 2.8$ & -0.14 & & & & \\
\hline HD 6963 & Field & 9.0 & 0.40 & $1.00 \pm 0.05$ & $1.06 \pm 0.02$ & $13.3 \pm 2.6$ & -7.72 & & $\mathrm{Y}$ & $\mathrm{Y}$ & $\mathrm{Y}$ \\
\hline HD 7661 & Field & 8.6 & 0.47 & $1.00 \pm 0.05$ & $0.99 \pm 0.02$ & $1.1 \pm 2.0$ & -1.28 & & & & \\
\hline HD 8941 & Field & 9.2 & 0.28 & $1.05 \pm 0.05$ & $0.99 \pm 0.02$ & $0.8 \pm 1.7$ & -0.07 & & & & \\
\hline HD 9472 & Field & 8.9 & 0.38 & $0.99 \pm 0.05$ & $1.00 \pm 0.02$ & $0.4 \pm 3.2$ & -0.81 & & & & \\
\hline HD 11850 & Field & 8.8 & 0.38 & $1.04 \pm 0.05$ & $1.01 \pm 0.02$ & $-1.6 \pm 3.7$ & -1.57 & & & & \\
\hline HD 12039 & Field & 7.5 & 0.38 & $1.10 \pm 0.05$ & $1.34 \pm 0.02$ & $1.7 \pm 3.9$ & -24.80 & & $\mathrm{Y}$ & $\mathrm{Y}$ & \\
\hline HD 13382 & Field & 8.7 & 0.33 & $1.01 \pm 0.05$ & $1.03 \pm 0.02$ & $0.8 \pm 2.5$ & -0.37 & & & & \\
\hline HD 13507 & Field & 8.9 & 0.38 & $1.02 \pm 0.05$ & $0.98 \pm 0.02$ & $0.9 \pm 1.6$ & -0.40 & & & & \\
\hline HD 13531 & Field & 8.7 & 0.40 & $1.00 \pm 0.05$ & $0.99 \pm 0.02$ & $0.4 \pm 1.9$ & -1.36 & & & & \\
\hline HD 13974 & Field & 9.2 & $\cdots$ & $0.95 \pm 0.05$ & $1.01 \pm 0.02$ & $1.1 \pm 0.2$ & -2.70 & & $\mathrm{Y}$ & & \\
\hline HD 15526 & Field & 7.6 & 0.43 & $1.01 \pm 0.05$ & $0.99 \pm 0.03$ & $-8.6 \pm 7.6$ & -1.59 & & & & \\
\hline HD 18940 & Field & 8.9 & 0.36 & $1.03 \pm 0.05$ & $1.00 \pm 0.02$ & $-0.9 \pm 2.0$ & -0.94 & & & & \\
\hline HD 19019 & Field & 9.2 & 0.31 & $0.98 \pm 0.05$ & $1.01 \pm 0.02$ & $0.9 \pm 2.3$ & -2.22 & & & & \\
\hline HD 19668 & Field & 8.4 & 0.46 & $1.12 \pm 0.05$ & $1.24 \pm 0.02$ & $-1.2 \pm 5.6$ & -15.91 & & $\mathrm{Y}$ & $\mathrm{Y}$ & \\
\hline HD 21411 & Field & 8.9 & 0.44 & $0.98 \pm 0.05$ & $0.99 \pm 0.02$ & $1.4 \pm 2.8$ & -1.17 & & & & \\
\hline HD 22179 & Field & 7.2 & 0.34 & $0.97 \pm 0.05$ & $1.33 \pm 0.03$ & $39.5 \pm 11.7$ & -47.54 & & $\mathrm{Y}$ & $\mathrm{Y}$ & $\mathrm{Y}$ \\
\hline HD 25300 & Field & 8.2 & 0.78 & $1.11 \pm 0.05$ & $1.01 \pm 0.02$ & $0.4 \pm 2.4$ & -1.00 & & & & \\
\hline HD 26182 & Field & 7.8 & 0.33 & $0.98 \pm 0.05$ & $0.97 \pm 0.03$ & $2.4 \pm 13.2$ & -1.44 & & & & \\
\hline HD 26990 & Field & 8.9 & 0.40 & $0.97 \pm 0.05$ & $1.01 \pm 0.02$ & $2.6 \pm 2.6$ & -0.17 & & & & \\
\hline HD 27466 & Field & 9.2 & 0.37 & $0.98 \pm 0.05$ & $0.98 \pm 0.02$ & $0.7 \pm 3.1$ & -1.59 & & & & \\
\hline HD 28495 & Field & 8.5 & 0.49 & $1.06 \pm 0.05$ & $1.02 \pm 0.02$ & $-1.7 \pm 2.5$ & -0.25 & & & & \\
\hline HD 29231 & Field & 9.2 & 0.44 & $1.02 \pm 0.05$ & $0.99 \pm 0.02$ & $1.0 \pm 2.0$ & -0.17 & & & & \\
\hline HD 31143 & Field & 9.1 & 0.50 & $1.01 \pm 0.05$ & $0.99 \pm 0.02$ & $1.1 \pm 3.1$ & -0.77 & & & & \\
\hline HD 31281 & Field & 6.8 & 0.34 & $0.94 \pm 0.05$ & $1.01 \pm 0.03$ & $12.0 \pm 16.8$ & -5.18 & & $\mathrm{Y}$ & & \\
\hline HD 31392 & Field & 9.1 & 0.48 & $0.99 \pm 0.05$ & $1.02 \pm 0.02$ & $20.8 \pm 2.6$ & -4.67 & & $\mathrm{Y}$ & & $\mathrm{Y}$ \\
\hline HD 31950 & Field & 7.8 & 0.27 & $1.00 \pm 0.05$ & $1.06 \pm 0.03$ & $-7.3 \pm 24.1$ & -1.68 & & & & \\
\hline HD 32850 & Field & 9.1 & 0.49 & $1.03 \pm 0.05$ & $1.01 \pm 0.02$ & $0.6 \pm 2.9$ & -0.26 & & & & \\
\hline HD 37006 & Field & 8.9 & 0.40 & $1.05 \pm 0.05$ & $1.01 \pm 0.02$ & $-1.1 \pm 3.1$ & -0.20 & & & & \\
\hline HD 37216 & Field & 8.8 & 0.42 & $1.04 \pm 0.05$ & $0.99 \pm 0.02$ & $2.2 \pm 3.0$ & -0.53 & & & & \\
\hline HD 37572 & Field & 7.8 & 0.56 & $1.06 \pm 0.05$ & $1.01 \pm 0.02$ & $1.5 \pm 2.0$ & -2.22 & & & & \\
\hline HD 37962 & Field & 9.2 & 0.41 & $1.03 \pm 0.05$ & $1.01 \pm 0.02$ & $6.8 \pm 3.1$ & -1.44 & & & & \\
\hline HD 38529 & Field & 9.7 & $\ldots$ & $0.95 \pm 0.05$ & $0.99 \pm 0.02$ & $4.6 \pm 0.8$ & -12.99 & & $\mathrm{Y}$ & & $\mathrm{Y}$ \\
\hline HD 38949 & Field & 8.3 & 0.31 & $1.03 \pm 0.05$ & $1.03 \pm 0.02$ & $3.4 \pm 3.8$ & -4.80 & & $\mathrm{Y}$ & & \\
\hline HD 40647 & Field & 8.8 & 0.48 & $1.05 \pm 0.05$ & $1.00 \pm 0.02$ & $2.9 \pm 2.8$ & -0.12 & & & & \\
\hline HD 43989 & Field & 7.8 & 0.30 & $1.04 \pm 0.05$ & $1.15 \pm 0.02$ & $3.5 \pm 4.9$ & -13.83 & & $\mathrm{Y}$ & $\mathrm{Y}$ & \\
\hline HD 44594 & Field & 9.8 & 0.40 & $1.01 \pm 0.05$ & $0.97 \pm 0.02$ & $0.7 \pm 0.9$ & -1.80 & & & & \\
\hline HD 45270 & Field & 7.9 & 0.39 & $1.04 \pm 0.05$ & $1.04 \pm 0.02$ & $1.2 \pm 0.8$ & -5.46 & & $\mathrm{Y}$ & & \\
\hline HD 47875 & Field & 7.8 & 0.47 & $1.07 \pm 0.05$ & $1.06 \pm 0.03$ & $0.0 \pm 3.7$ & -0.58 & & & & \\
\hline HD 60737 & Field & 8.2 & 0.33 & $1.03 \pm 0.05$ & $1.06 \pm 0.02$ & $7.2 \pm 4.5$ & -3.57 & & $\mathrm{Y}$ & $\mathrm{Y}$ & \\
\hline HD 61005 & Field & 8.1 & 0.45 & $1.11 \pm 0.05$ & $2.18 \pm 0.02$ & $303.9 \pm 22.9$ & -90.66 & & $\mathrm{Y}$ & $\mathrm{Y}$ & $\mathrm{Y}$ \\
\hline HD 61994 & Field & 9.0 & 0.43 & $0.99 \pm 0.05$ & $0.98 \pm 0.02$ & $1.2 \pm 1.2$ & -0.24 & & & & \\
\hline HD 64324 & Field & 9.1 & 0.35 & $1.00 \pm 0.05$ & $1.02 \pm 0.02$ & $2.5 \pm 3.9$ & -0.95 & & & & \\
\hline HD 66751 & Field & 9.2 & 0.34 & $0.97 \pm 0.05$ & $0.98 \pm 0.02$ & $0.8 \pm 1.0$ & -0.08 & & & & \\
\hline
\end{tabular}


Table 3

(Continued)

\begin{tabular}{|c|c|c|c|c|c|c|c|c|c|c|c|c|}
\hline \multirow[t]{2}{*}{ Name } & \multirow{2}{*}{$\begin{array}{l}\text { Stellar } \\
\text { Group }^{\mathrm{a}}\end{array}$} & \multirow[t]{2}{*}{ log (age/years) } & \multirow{2}{*}{$\begin{array}{c}\left(J-K_{\mathrm{S}}\right)_{\mathrm{o}}{ }^{\mathrm{b}} \\
(\mathrm{mag})\end{array}$} & \multicolumn{3}{|c|}{ Normalized Ratios $^{\mathrm{c}}$} & \multirow[t]{2}{*}{$\log p^{\mathrm{d}}$} & \multicolumn{5}{|c|}{ Excess? } \\
\hline & & & & $R_{16 / 8}$ & $R_{24 / 8}$ & $R_{70 / 8}$ & & $8 \mu \mathrm{m}$ & $16 \mu \mathrm{m}$ & IRS & $24 \mu \mathrm{m}$ & $70 \mu \mathrm{m}$ \\
\hline HD 69076 & Field & 9.3 & 0.45 & $1.02 \pm 0.05$ & $1.01 \pm 0.02$ & $0.2 \pm 3.9$ & -0.05 & & & & & \\
\hline HD 70516 & Field & 7.9 & 0.37 & $1.02 \pm 0.05$ & $0.99 \pm 0.02$ & $3.0 \pm 2.5$ & -0.87 & & & & & \\
\hline HD 70573 & Field & 8.0 & 0.37 & $0.97 \pm 0.05$ & $1.02 \pm 0.03$ & $13.4 \pm 5.3$ & -3.03 & & & $\mathrm{Y}$ & & \\
\hline HD 71974 & Field & 8.9 & 0.45 & $0.99 \pm 0.05$ & $1.00 \pm 0.02$ & $3.2 \pm 1.5$ & -0.79 & & & & & \\
\hline HD 72687 & Field & 8.4 & 0.34 & $1.06 \pm 0.05$ & $1.26 \pm 0.02$ & $2.0 \pm 5.4$ & -26.32 & & & $\mathrm{Y}$ & $\mathrm{Y}$ & \\
\hline HD 73668 & Field & 9.3 & 0.36 & $1.02 \pm 0.05$ & $1.00 \pm 0.02$ & $2.7 \pm 2.7$ & -0.16 & & & & & \\
\hline HD 75302 & Field & 9.1 & 0.40 & $1.01 \pm 0.05$ & $0.99 \pm 0.02$ & $0.4 \pm 2.4$ & -0.29 & & & & & \\
\hline HD 75393 & Field & 8.4 & 0.33 & $1.01 \pm 0.05$ & $1.00 \pm 0.02$ & $-1.8 \pm 2.6$ & -0.87 & & & & & \\
\hline HD 76218 & Field & 8.7 & 0.43 & $1.03 \pm 0.05$ & $0.99 \pm 0.02$ & $-1.4 \pm 2.7$ & -0.24 & & & & & \\
\hline HD 77407 & Field & 7.5 & 0.36 & $1.03 \pm 0.05$ & $0.98 \pm 0.02$ & $2.0 \pm 1.7$ & -1.31 & & & & & \\
\hline HD 80606 & Field & 9.7 & 0.39 & $\ldots$ & $0.97 \pm 0.03$ & $3.6 \pm 5.3$ & $\ldots$ & & & & & \\
\hline HD 85301 & Field & 8.7 & 0.37 & $1.12 \pm 0.05$ & $1.39 \pm 0.02$ & $13.4 \pm 2.6$ & -42.12 & & & $\mathrm{Y}$ & $\mathrm{Y}$ & $\mathrm{Y}$ \\
\hline HD 86356 & Field & 7.8 & 0.46 & $0.94 \pm 0.05$ & $0.99 \pm 0.03$ & $-4.7 \pm 7.9$ & -0.94 & & & & & \\
\hline HD 88201 & Field & 8.5 & 0.28 & $1.01 \pm 0.05$ & $0.99 \pm 0.02$ & $-0.4 \pm 2.6$ & -0.88 & & & & & \\
\hline HD 88742 & Field & 9.3 & 0.37 & $1.02 \pm 0.05$ & $0.98 \pm 0.02$ & $1.0 \pm 1.1$ & -0.39 & & & & & \\
\hline HD 90712 & Field & 8.3 & 0.37 & $1.02 \pm 0.05$ & $0.99 \pm 0.02$ & $-0.9 \pm 2.8$ & -0.33 & & & & & \\
\hline HD 90905 & Field & 8.2 & 0.33 & $1.00 \pm 0.05$ & $1.10 \pm 0.02$ & $4.5 \pm 2.4$ & -9.23 & & & $\mathrm{Y}$ & $\mathrm{Y}$ & \\
\hline HD 91782 & Field & 8.2 & 0.30 & $1.04 \pm 0.05$ & $1.03 \pm 0.02$ & $-1.2 \pm 4.8$ & -0.16 & & & & & \\
\hline HD 91962 & Field & 8.4 & 0.45 & $1.00 \pm 0.05$ & $0.98 \pm 0.02$ & $-0.1 \pm 1.4$ & -0.73 & & & & & \\
\hline HD 92788 & Field & 9.7 & 0.41 & $0.98 \pm 0.05$ & $0.99 \pm 0.02$ & $2.8 \pm 2.3$ & -0.50 & & & & & \\
\hline HD 92855 & Field & 8.2 & 0.39 & $1.01 \pm 0.05$ & $1.00 \pm 0.02$ & $2.8 \pm 1.9$ & -0.60 & & & & & \\
\hline HD 95188 & Field & 8.4 & 0.45 & $0.99 \pm 0.05$ & $1.01 \pm 0.02$ & $3.6 \pm 5.6$ & -0.37 & & & & & \\
\hline HD 98553 & Field & 9.2 & 0.35 & $1.01 \pm 0.05$ & $0.98 \pm 0.02$ & $1.3 \pm 2.8$ & -0.57 & & & & & \\
\hline HD 100167 & Field & 9.2 & 0.36 & $1.01 \pm 0.05$ & $0.99 \pm 0.02$ & $-0.8 \pm 2.3$ & -0.18 & & & & & \\
\hline HD 101472 & Field & 8.4 & 0.32 & $1.02 \pm 0.05$ & $1.00 \pm 0.02$ & $-0.2 \pm 3.1$ & -0.92 & & & & & \\
\hline HD 101959 & Field & 9.2 & 0.35 & $1.01 \pm 0.05$ & $0.99 \pm 0.02$ & $2.2 \pm 1.9$ & -1.82 & & & & & \\
\hline HD 102071 & Field & 9.3 & 0.49 & $1.02 \pm 0.05$ & $1.00 \pm 0.02$ & $3.2 \pm 2.5$ & -0.24 & & & & & \\
\hline HD 103432 & Field & 9.3 & 0.41 & $0.97 \pm 0.05$ & $0.99 \pm 0.02$ & $4.5 \pm 5.3$ & -0.81 & & & & & \\
\hline HD 104467 & Field & 6.7 & 0.39 & $1.04 \pm 0.05$ & $1.03 \pm 0.02$ & $-1.3 \pm 3.3$ & -2.70 & & & & & \\
\hline HD 104576 & Field & 8.2 & 0.46 & $0.97 \pm 0.05$ & $1.01 \pm 0.02$ & $4.0 \pm 4.6$ & -1.77 & & & & & \\
\hline HD 104860 & Field & 7.6 & 0.32 & $1.03 \pm 0.05$ & $1.09 \pm 0.02$ & $92.2 \pm 7.7$ & -16.50 & & & $\mathrm{Y}$ & $\mathrm{Y}$ & $\mathrm{Y}$ \\
\hline HD 105631 & Field & 9.2 & 0.43 & $1.00 \pm 0.05$ & $0.99 \pm 0.02$ & $0.2 \pm 1.7$ & -0.12 & & & & & \\
\hline HD 106156 & Field & 9.3 & 0.45 & $1.01 \pm 0.05$ & $0.99 \pm 0.02$ & $5.4 \pm 3.4$ & -1.31 & & & & & \\
\hline HD 106252 & Field & 9.5 & 0.37 & $1.00 \pm 0.05$ & $1.00 \pm 0.02$ & $5.0 \pm 2.9$ & -1.54 & & & & & \\
\hline HD 106772 & Field & 8.8 & 0.33 & $1.00 \pm 0.05$ & $1.01 \pm 0.02$ & $-1.6 \pm 2.4$ & -0.18 & & & & & \\
\hline HD 107146 & Field & 8.2 & 0.33 & $1.01 \pm 0.05$ & $1.38 \pm 0.02$ & $142.4 \pm 10.6$ & -66.32 & & & $\mathrm{Y}$ & $\mathrm{Y}$ & $\mathrm{Y}$ \\
\hline HD 107441 & LCC & 7.2 & 0.42 & $0.99 \pm 0.05$ & $1.05 \pm 0.03$ & $2.1 \pm 9.6$ & -2.00 & & & & & \\
\hline HD 108799 & Field & 8.3 & 0.41 & $1.03 \pm 0.05$ & $0.99 \pm 0.02$ & $0.5 \pm 1.1$ & -0.35 & & & & & \\
\hline HD 108944 & Field & 8.2 & 0.27 & $1.04 \pm 0.05$ & $1.00 \pm 0.02$ & $0.5 \pm 2.7$ & -1.37 & & & & & \\
\hline HD 111170 & LCC & 7.2 & 0.47 & $0.96 \pm 0.05$ & $1.01 \pm 0.03$ & $-4.5 \pm 8.3$ & -1.30 & & & & & \\
\hline HD 112196 & Field & 7.9 & 0.33 & $1.02 \pm 0.05$ & $0.98 \pm 0.02$ & $0.7 \pm 1.9$ & -0.91 & & & & & \\
\hline HD 115043 & Field & 8.7 & 0.34 & $0.99 \pm 0.05$ & $0.99 \pm 0.02$ & $-0.1 \pm 1.3$ & -1.14 & & & & & \\
\hline HD 116099 & LCC & 7.2 & 0.39 & $1.04 \pm 0.05$ & $1.15 \pm 0.03$ & $10.5 \pm 10.2$ & -13.96 & & & $\mathrm{Y}$ & $\mathrm{Y}$ & \\
\hline HD 117524 & LCC & 7.2 & 0.47 & $1.02 \pm 0.05$ & $0.99 \pm 0.03$ & $-15.4 \pm 15.1$ & -0.64 & & & & & \\
\hline HD 119269 & LCC & 7.2 & 0.36 & $1.02 \pm 0.05$ & $1.02 \pm 0.03$ & $27.4 \pm 13.5$ & -6.89 & & & $\mathrm{Y}$ & & \\
\hline HD 120812 & UCL & 7.2 & 0.40 & $1.00 \pm 0.05$ & $1.00 \pm 0.03$ & $-15.1 \pm 13.4$ & -0.66 & & & & & \\
\hline HD 121320 & Field & 9.3 & 0.35 & $0.98 \pm 0.05$ & $0.97 \pm 0.02$ & $0.8 \pm 3.8$ & -0.10 & & & & & \\
\hline HD 121504 & Field & 9.3 & 0.43 & $1.04 \pm 0.05$ & $0.99 \pm 0.02$ & $9.9 \pm 6.8$ & -2.30 & & & & & \\
\hline HD 122652 & Field & 9.3 & 0.28 & $1.03 \pm 0.05$ & $1.10 \pm 0.02$ & $24.0 \pm 3.2$ & -7.98 & & & $\mathrm{Y}$ & $\mathrm{Y}$ & $\mathrm{Y}$ \\
\hline HD 126670 & UCL & 7.2 & 0.46 & $1.00 \pm 0.05$ & $1.05 \pm 0.03$ & $-1.3 \pm 9.0$ & -2.05 & & & & & \\
\hline HD 128242 & UCL & 7.2 & 0.38 & $1.01 \pm 0.05$ & $1.01 \pm 0.03$ & $-9.4 \pm 8.7$ & -1.27 & & & & & \\
\hline HD 129333 & Field & 7.9 & 0.40 & $1.01 \pm 0.05$ & $1.02 \pm 0.02$ & $1.8 \pm 1.8$ & -0.31 & & & & & \\
\hline HD 132173 & Field & 8.2 & 0.36 & $1.04 \pm 0.05$ & $1.04 \pm 0.02$ & $-0.3 \pm 4.0$ & -3.00 & & & $\mathrm{Y}$ & & \\
\hline HD 133295 & Field & 8.5 & 0.34 & $1.04 \pm 0.05$ & $1.01 \pm 0.02$ & $3.3 \pm 3.4$ & -0.29 & & & & & \\
\hline HD 133938 & UCL & 7.2 & 0.43 & $0.94 \pm 0.05$ & $0.97 \pm 0.03$ & $-13.3 \pm 30.4$ & -0.79 & & & & & \\
\hline HD 135363 & Field & 7.8 & 0.64 & $1.06 \pm 0.05$ & $1.04 \pm 0.02$ & $0.1 \pm 2.4$ & -3.43 & & & $\mathrm{Y}$ & & \\
\hline HD 136923 & Field & 9.3 & 0.42 & $1.00 \pm 0.05$ & $0.98 \pm 0.02$ & $1.9 \pm 1.3$ & -1.96 & & & & & \\
\hline HD 138004 & Field & 9.3 & 0.36 & $1.01 \pm 0.05$ & $0.98 \pm 0.02$ & $0.5 \pm 2.2$ & -0.84 & & & & & \\
\hline HD 139498 & UCL & 7.2 & 0.45 & $0.94 \pm 0.05$ & $1.01 \pm 0.03$ & $-8.7 \pm 18.4$ & -1.46 & & & & & \\
\hline HD 139813 & Field & 8.3 & 0.42 & $\ldots$ & $0.98 \pm 0.02$ & $3.0 \pm 1.5$ & $\ldots$ & & & & & \\
\hline HD 140374 & UCL & 7.2 & 0.45 & $0.88 \pm 0.05$ & $0.95 \pm 0.03$ & $-19.0 \pm 18.1$ & -7.55 & & & & & \\
\hline HD 141521 & UCL & 7.2 & 0.47 & $0.98 \pm 0.05$ & $1.01 \pm 0.03$ & $-14.7 \pm 17.3$ & -1.66 & & & & & \\
\hline HD 141937 & Field & 9.4 & 0.36 & $1.01 \pm 0.05$ & $1.00 \pm 0.02$ & $-0.7 \pm 3.0$ & -1.08 & & & & & \\
\hline HD 141943 & Field & 7.2 & 0.40 & $1.03 \pm 0.05$ & $1.26 \pm 0.02$ & $15.9 \pm 6.5$ & -39.34 & & & Y & Y & \\
\hline
\end{tabular}


Table 3

(Continued)

\begin{tabular}{|c|c|c|c|c|c|c|c|c|c|c|c|c|}
\hline \multirow[t]{2}{*}{ Name } & \multirow{2}{*}{$\begin{array}{l}\text { Stellar } \\
\text { Group }^{\mathrm{a}}\end{array}$} & \multirow[t]{2}{*}{$\log$ (age/years) } & \multirow{2}{*}{$\begin{array}{c}\left(J-K_{\mathrm{S}}\right)_{\mathrm{o}}{ }^{\mathrm{b}} \\
(\mathrm{mag})\end{array}$} & \multicolumn{3}{|c|}{ Normalized Ratios $^{\mathrm{c}}$} & \multirow[t]{2}{*}{$\log p^{d}$} & \multicolumn{5}{|c|}{ Excess? } \\
\hline & & & & $R_{16 / 8}$ & $R_{24 / 8}$ & $R_{70 / 8}$ & & $8 \mu \mathrm{m}$ & $16 \mu \mathrm{m}$ & IRS & $24 \mu \mathrm{m}$ & $70 \mu \mathrm{m}$ \\
\hline HD 142229 & Field & 8.8 & 0.36 & $1.06 \pm 0.05$ & $1.03 \pm 0.02$ & $1.5 \pm 4.5$ & -0.09 & & & & & \\
\hline HD 142361 & USco & 6.7 & 0.46 & $1.01 \pm 0.05$ & $1.05 \pm 0.02$ & $9.2 \pm 14.7$ & -2.15 & & & & & \\
\hline HD 143358 & UCL & 7.2 & 0.39 & $1.01 \pm 0.05$ & $1.08 \pm 0.03$ & $4.0 \pm 28.9$ & -7.24 & & & $\mathrm{Y}$ & & \\
\hline HD 145229 & Field & 8.8 & 0.33 & $0.99 \pm 0.05$ & $1.08 \pm 0.02$ & $20.7 \pm 2.8$ & -15.17 & & & $\mathrm{Y}$ & $\mathrm{Y}$ & $\mathrm{Y}$ \\
\hline HD 146516 & USco & 6.7 & 0.41 & $0.99 \pm 0.05$ & $1.02 \pm 0.03$ & $6.7 \pm 18.8$ & -0.01 & & & & & \\
\hline HD 150554 & Field & 9.4 & $\cdots$ & $1.01 \pm 0.05$ & $1.00 \pm 0.02$ & $-3.0 \pm 3.1$ & -0.27 & & & & & \\
\hline HD 150706 & Field & 8.8 & 0.33 & $1.02 \pm 0.05$ & $1.03 \pm 0.02$ & $8.7 \pm 1.8$ & -4.38 & & & $\mathrm{Y}$ & & $\mathrm{Y}$ \\
\hline HD 151798 & Field & 7.8 & 0.34 & $0.99 \pm 0.05$ & $0.99 \pm 0.02$ & $-2.5 \pm 8.3$ & -0.34 & & & & & \\
\hline HD 152555 & Field & 8.1 & 0.34 & $0.95 \pm 0.05$ & $0.99 \pm 0.02$ & $-0.2 \pm 4.2$ & -0.36 & & & & & \\
\hline HD 153458 & Field & 9.2 & 0.35 & $0.95 \pm 0.05$ & $1.02 \pm 0.02$ & $-2.7 \pm 5.8$ & -1.74 & & & & & \\
\hline HD 154417 & Field & 9.1 & $\ldots$ & $0.94 \pm 0.05$ & $1.00 \pm 0.02$ & $0.5 \pm 0.8$ & -2.22 & & & & & \\
\hline HD 157664 & Field & 9.6 & 0.25 & $1.02 \pm 0.05$ & $1.00 \pm 0.02$ & $1.9 \pm 4.9$ & -0.84 & & & & & \\
\hline HD 159222 & Field & 9.3 & 0.34 & $0.99 \pm 0.05$ & $0.98 \pm 0.02$ & $0.8 \pm 1.0$ & -1.77 & & & & & \\
\hline HD 161897 & Field & 9.3 & 0.37 & $1.04 \pm 0.05$ & $0.98 \pm 0.02$ & $-0.6 \pm 3.5$ & -1.15 & & & & & \\
\hline HD 167389 & Field & 9.3 & 0.31 & $1.02 \pm 0.05$ & $0.99 \pm 0.02$ & $-1.3 \pm 2.8$ & -0.58 & & & & & \\
\hline HD 170778 & Field & 8.6 & 0.36 & $1.05 \pm 0.05$ & $1.00 \pm 0.02$ & $1.3 \pm 2.1$ & -0.06 & & & & & \\
\hline HD 172649 & Field & 8.2 & 0.25 & $1.02 \pm 0.05$ & $1.01 \pm 0.02$ & $-0.5 \pm 3.0$ & -0.78 & & & & & \\
\hline HD 174656 & $\mathrm{CrA}$ & 6.5 & 0.48 & $1.01 \pm 0.05$ & $1.04 \pm 0.03$ & $1.5 \pm 4.2$ & -1.18 & & & & & \\
\hline HD 179949 & Field & 9.3 & 0.36 & $0.94 \pm 0.05$ & $1.00 \pm 0.02$ & $-0.6 \pm 1.3$ & -0.12 & & & & & \\
\hline HD 183216 & Field & 9.1 & 0.31 & $0.97 \pm 0.05$ & $1.09 \pm 0.02$ & $5.8 \pm 2.6$ & -5.38 & & & $\mathrm{Y}$ & $\mathrm{Y}$ & \\
\hline HD 187897 & Field & 9.1 & 0.38 & $0.94 \pm 0.05$ & $1.04 \pm 0.02$ & $14.9 \pm 2.3$ & -0.74 & & & & & $\mathrm{Y}$ \\
\hline HD 190228 & Field & 10.0 & 0.47 & $1.03 \pm 0.05$ & $1.00 \pm 0.02$ & $2.0 \pm 4.5$ & -0.09 & & & & & \\
\hline HD 193017 & Field & 9.2 & 0.29 & $1.01 \pm 0.05$ & $1.00 \pm 0.02$ & $2.0 \pm 2.9$ & -0.61 & & & & & \\
\hline HD 195034 & Field & 9.3 & 0.32 & $1.05 \pm 0.05$ & $0.98 \pm 0.02$ & $0.4 \pm 1.8$ & -2.05 & & & & & \\
\hline HD 199019 & Field & 8.4 & 0.39 & $1.06 \pm 0.05$ & $1.02 \pm 0.02$ & $4.5 \pm 3.9$ & -2.00 & & & & & \\
\hline HD 199143 & Field & 7.2 & 0.40 & $1.08 \pm 0.05$ & $1.04 \pm 0.02$ & $2.4 \pm 2.8$ & -1.31 & & & & & \\
\hline HD 199598 & Field & 9.2 & 0.31 & $1.02 \pm 0.05$ & $1.00 \pm 0.02$ & $1.4 \pm 1.6$ & -0.50 & & & & & \\
\hline HD 200746 & Field & 8.6 & 0.40 & $1.07 \pm 0.05$ & $1.01 \pm 0.02$ & $5.1 \pm 3.6$ & -1.00 & & & & & \\
\hline HD 201219 & Field & 9.0 & 0.40 & $1.06 \pm 0.05$ & $1.08 \pm 0.02$ & $19.1 \pm 3.5$ & -6.46 & & & $\mathrm{Y}$ & $\mathrm{Y}$ & $\mathrm{Y}$ \\
\hline HD 201989 & Field & 9.0 & 0.41 & $1.04 \pm 0.05$ & $0.99 \pm 0.02$ & $-0.0 \pm 2.4$ & -1.13 & & & & & \\
\hline HD 202108 & Field & 9.2 & 0.39 & $1.00 \pm 0.05$ & $0.99 \pm 0.02$ & $-0.1 \pm 2.4$ & -1.54 & & & & & \\
\hline HD 203030 & Field & 8.3 & 0.41 & $1.06 \pm 0.05$ & $1.01 \pm 0.02$ & $3.9 \pm 4.4$ & -0.18 & & & & & \\
\hline HD 204277 & Field & 8.7 & 0.26 & $1.03 \pm 0.05$ & $1.04 \pm 0.02$ & $5.8 \pm 2.1$ & -7.13 & & & $\mathrm{Y}$ & & \\
\hline HD 205905 & Field & 9.1 & 0.37 & $1.01 \pm 0.05$ & $0.98 \pm 0.02$ & $2.9 \pm 1.5$ & -0.21 & & & & & \\
\hline HD 206374 & Field & 9.2 & 0.37 & $0.96 \pm 0.05$ & $1.00 \pm 0.02$ & $4.8 \pm 1.8$ & -2.40 & & & & & \\
\hline HD 209393 & Field & 8.6 & 0.39 & $0.94 \pm 0.05$ & $1.00 \pm 0.02$ & $-1.0 \pm 3.8$ & -0.41 & & & & & \\
\hline HD 209779 & Field & 8.6 & 0.44 & $1.05 \pm 0.05$ & $1.00 \pm 0.02$ & $2.8 \pm 3.2$ & -0.45 & & & & & \\
\hline HD 212291 & Field & 9.3 & 0.37 & $0.99 \pm 0.05$ & $0.98 \pm 0.02$ & $3.2 \pm 3.0$ & -0.61 & & & & & \\
\hline HD 216275 & Field & 9.3 & 0.31 & $0.99 \pm 0.05$ & $0.99 \pm 0.02$ & $1.9 \pm 2.8$ & -1.41 & & & & & \\
\hline HD 217343 & Field & 7.6 & 0.35 & $1.06 \pm 0.05$ & $1.03 \pm 0.02$ & $-0.2 \pm 2.7$ & -0.88 & & & & & \\
\hline HD 219498 & Field & 8.6 & 0.38 & $1.01 \pm 0.05$ & $1.27 \pm 0.03$ & $25.4 \pm 4.5$ & -34.85 & & & $\mathrm{Y}$ & $\mathrm{Y}$ & $\mathrm{Y}$ \\
\hline HD 224873 & Field & 8.5 & 0.45 & $1.02 \pm 0.05$ & $0.98 \pm 0.02$ & $-3.9 \pm 4.9$ & -0.51 & & & & & \\
\hline HD 245567 & Field & 6.6 & 0.41 & $0.88 \pm 0.05$ & $0.96 \pm 0.03$ & $-32.4 \pm 21.1$ & -3.35 & & & & & \\
\hline HD 279788 & Field & 6.6 & 0.41 & $0.94 \pm 0.05$ & $1.09 \pm 0.03$ & $7.4 \pm 32.8$ & -1.92 & & & & & \\
\hline HD 281691 & Field & 7.1 & 0.53 & $0.99 \pm 0.05$ & $1.12 \pm 0.03$ & $-4.7 \pm 15.5$ & -3.30 & & & $\mathrm{Y}$ & $\mathrm{Y}$ & \\
\hline HD 282346 & Field & 8.0 & 0.59 & $0.99 \pm 0.05$ & $0.99 \pm 0.03$ & $-22.6 \pm 11.6$ & -1.02 & & & & & \\
\hline HD 284135 & Field & 6.8 & 0.35 & $0.97 \pm 0.05$ & $1.01 \pm 0.03$ & $11.4 \pm 8.5$ & -5.91 & & & $\mathrm{Y}$ & & \\
\hline HD 284266 & Field & 7.2 & 0.54 & $0.98 \pm 0.05$ & $1.04 \pm 0.03$ & $-9.1 \pm 39.6$ & -1.06 & & & & & \\
\hline HD 285281 & Field & 7.0 & 0.54 & $0.95 \pm 0.05$ & $1.02 \pm 0.03$ & $-2.2 \pm 5.5$ & -0.99 & & & & & \\
\hline HD 285372 & Field & 6.8 & 0.63 & $1.02 \pm 0.05$ & $0.98 \pm 0.03$ & $-16.1 \pm 34.0$ & -3.00 & & & $\mathrm{Y}$ & & \\
\hline HD 285751 & Field & 6.8 & 0.55 & $0.96 \pm 0.05$ & $1.05 \pm 0.03$ & $-8.1 \pm 41.4$ & -3.00 & & & & & \\
\hline HD 285840 & Field & 7.8 & 0.54 & $0.97 \pm 0.05$ & $1.01 \pm 0.03$ & $-10.3 \pm 25.0$ & -1.52 & & & & & \\
\hline HD 286179 & Field & 7.2 & 0.38 & $0.88 \pm 0.05$ & $0.97 \pm 0.03$ & $-12.7 \pm 25.8$ & -0.21 & & & & & \\
\hline HD 286264 & Field & 7.3 & 0.59 & $0.94 \pm 0.05$ & $1.03 \pm 0.03$ & $-8.4 \pm 9.9$ & -1.74 & & & & & \\
\hline HE 350 & $\alpha$ Per & 7.9 & 0.36 & $0.97 \pm 0.05$ & $1.05 \pm 0.03$ & $36.3 \pm 59.5$ & -0.47 & & & & & \\
\hline HE 373 & $\alpha$ Per & 7.9 & 0.43 & $0.93 \pm 0.05$ & $1.00 \pm 0.03$ & $-20.3 \pm 30.6$ & -9.67 & & & & & \\
\hline HE 389 & $\alpha$ Per & 7.9 & 0.33 & $0.95 \pm 0.05$ & $0.97 \pm 0.03$ & $-1.0 \pm 77.7$ & -0.97 & & & & & \\
\hline HE 622 & $\alpha$ Per & 7.9 & 0.46 & $0.88 \pm 0.05$ & $0.99 \pm 0.03$ & $14.2 \pm 82.0$ & -2.22 & & & & & \\
\hline HE 696 & $\alpha$ Per & 7.9 & 0.43 & $0.85 \pm 0.05$ & $0.98 \pm 0.03$ & $-175.5 \pm 96.0$ & -4.98 & & & & & \\
\hline HE 699 & $\alpha$ Per & 7.9 & 0.43 & $\cdots$ & $0.99 \pm 0.03$ & $26.2 \pm 37.8$ & $\ldots$ & & & & & \\
\hline HE 750 & $\alpha$ Per & 7.9 & 0.24 & $0.92 \pm 0.05$ & $1.18 \pm 0.03$ & $-78.0 \pm 57.4$ & -4.07 & & & $\mathrm{Y}$ & $\mathrm{Y}$ & \\
\hline HE 767 & $\alpha$ Per & 7.9 & 0.27 & $0.91 \pm 0.05$ & $0.99 \pm 0.03$ & $-0.2 \pm 37.0$ & -1.66 & & & & & \\
\hline
\end{tabular}


Table 3

(Continued)

\begin{tabular}{|c|c|c|c|c|c|c|c|c|c|c|c|c|}
\hline \multirow[t]{2}{*}{ Name } & \multirow{2}{*}{$\begin{array}{l}\text { Stellar } \\
\text { Group }^{\mathrm{a}} \\
\end{array}$} & \multirow{2}{*}{\multicolumn{2}{|c|}{$\begin{array}{c}\log (\text { age/years })\left(J-K_{\mathrm{s}}\right)_{\mathrm{o}}{ }^{\mathrm{b}} \\
(\mathrm{mag})\end{array}$}} & \multicolumn{3}{|c|}{ Normalized Ratios $^{\mathrm{c}}$} & \multirow[t]{2}{*}{$\log p^{\mathrm{d}}$} & \multicolumn{5}{|c|}{ Excess? } \\
\hline & & & & $R_{16 / 8}$ & $R_{24 / 8}$ & $R_{70 / 8}$ & & $8 \mu \mathrm{m}$ & $16 \mu \mathrm{m}$ & IRS & $24 \mu \mathrm{m}$ & $70 \mu \mathrm{m}$ \\
\hline HE 848 & $\alpha$ Per & 7.9 & 0.24 & $0.97 \pm 0.05$ & $1.49 \pm 0.03$ & $-24.2 \pm 35.9$ & -18.77 & & & $\mathrm{Y}$ & $\mathrm{Y}$ & \\
\hline HE 935 & $\alpha$ Per & 7.9 & 0.35 & $0.97 \pm 0.05$ & $1.00 \pm 0.03$ & $-24.6 \pm 25.4$ & -0.19 & & & & & \\
\hline HE 1101 & $\alpha$ Per & 7.9 & 0.42 & $0.88 \pm 0.05$ & $0.98 \pm 0.03$ & $-59.4 \pm 63.4$ & -3.21 & & & & & \\
\hline HE 1234 & $\alpha$ Per & 7.9 & 0.38 & $0.81 \pm 0.05$ & $0.97 \pm 0.03$ & $-45.0 \pm 80.0$ & -5.18 & & & & & \\
\hline H II 120 & Pleiades & 8.1 & 0.40 & $1.02 \pm 0.05$ & $1.00 \pm 0.03$ & $-59.8 \pm 86.2$ & -8.37 & & & & & \\
\hline H II 152 & Pleiades & 8.1 & 0.41 & $1.09 \pm 0.05$ & $1.34 \pm 0.03$ & $64.6 \pm 73.8$ & -8.90 & & & Y & Y & \\
\hline H II 173 & Pleiades & 8.1 & 0.48 & $0.94 \pm 0.05$ & $1.00 \pm 0.03$ & $-20.1 \pm 44.4$ & -0.45 & & & & & \\
\hline H II 174 & Pleiades & 8.1 & 0.56 & $0.92 \pm 0.05$ & $1.01 \pm 0.03$ & $31.0 \pm 73.7$ & -14.54 & & & & & \\
\hline H II 250 & Pleiades & 8.1 & 0.42 & $1.01 \pm 0.05$ & $1.13 \pm 0.03$ & $21.5 \pm 60.9$ & -3.04 & & & $\mathrm{Y}$ & $\mathrm{Y}$ & \\
\hline H II 314 & Pleiades & 8.1 & 0.42 & $1.03 \pm 0.05$ & $1.03 \pm 0.03$ & $13.9 \pm 62.1$ & -2.30 & & & & & \\
\hline H II 514 & Pleiades & 8.1 & 0.37 & $1.04 \pm 0.05$ & $1.23 \pm 0.03$ & $48.8 \pm 46.1$ & -11.53 & & & $\mathrm{Y}$ & $\mathrm{Y}$ & \\
\hline H II 1015 & Pleiades & 8.1 & 0.35 & $\ldots$ & $1.00 \pm 0.03$ & $-115.3 \pm 53.8$ & $\ldots$ & & & & & \\
\hline H II 1101 & Pleiades & 8.1 & 0.34 & $1.12 \pm 0.05$ & $1.52 \pm 0.03$ & $68.3 \pm 53.7$ & -30.26 & & & $\mathrm{Y}$ & $\mathrm{Y}$ & \\
\hline H II 1182 & Pleiades & 8.1 & 0.36 & $1.04 \pm 0.05$ & $1.01 \pm 0.03$ & $-18.4 \pm 24.3$ & -0.80 & & & & & \\
\hline H II 1200 & Pleiades & 8.1 & 0.30 & $0.99 \pm 0.05$ & $1.14 \pm 0.03$ & $-69.5 \pm 48.9$ & -7.26 & & & $\mathrm{Y}$ & $\mathrm{Y}$ & \\
\hline H II 1776 & Pleiades & 8.1 & 0.41 & $0.90 \pm 0.05$ & $1.02 \pm 0.03$ & $-33.4 \pm 61.3$ & -15.65 & & & & & \\
\hline H II 2147 & Pleiades & 8.1 & 0.54 & $1.00 \pm 0.05$ & $1.01 \pm 0.03$ & $-14.4 \pm 18.2$ & -1.19 & & & & & \\
\hline H II 2278 & Pleiades & 8.1 & 0.46 & $0.87 \pm 0.05$ & $1.00 \pm 0.03$ & $-56.6 \pm 38.0$ & -3.41 & & & & & \\
\hline Н II 2506 & Pleiades & 8.1 & 0.33 & $0.96 \pm 0.05$ & $1.02 \pm 0.03$ & $23.3 \pm 19.1$ & -0.66 & & & & & \\
\hline H II 2644 & Pleiades & 8.1 & 0.42 & $0.93 \pm 0.05$ & $0.99 \pm 0.03$ & $24.9 \pm 43.0$ & -0.97 & & & & & \\
\hline H II 2786 & Pleiades & 8.1 & 0.31 & $0.96 \pm 0.05$ & $0.98 \pm 0.03$ & $8.4 \pm 41.2$ & -0.98 & & & & & \\
\hline H II 2881 & Pleiades & 8.1 & 0.60 & $\ldots$ & $0.95 \pm 0.03$ & $40.8 \pm 29.8$ & $\ldots$ & & & & & \\
\hline H II 3097 & Pleiades & 8.1 & 0.38 & $0.97 \pm 0.05$ & $1.02 \pm 0.03$ & $-29.7 \pm 26.0$ & -0.63 & & & & & \\
\hline H II 3179 & Pleiades & 8.1 & 0.33 & $0.99 \pm 0.05$ & $1.00 \pm 0.03$ & $-12.9 \pm 13.7$ & -0.49 & & & & & \\
\hline HIP 6276 & Field & 8.5 & 0.48 & $1.04 \pm 0.05$ & $1.10 \pm 0.02$ & $7.2 \pm 5.7$ & -11.45 & & & $\mathrm{Y}$ & $\mathrm{Y}$ & \\
\hline HIP 42491 & Field & 9.2 & 0.45 & $\ldots$ & $0.99 \pm 0.02$ & $-4.2 \pm 6.2$ & $\ldots$ & & & & & \\
\hline HIP 59154 & Field & 8.6 & 0.58 & $1.03 \pm 0.05$ & $1.03 \pm 0.02$ & $0.7 \pm 4.4$ & -1.10 & & & & & \\
\hline HIP 76477 & UCL & 7.2 & 0.60 & $0.93 \pm 0.05$ & $1.01 \pm 0.03$ & $-1.9 \pm 19.2$ & -0.35 & & & & & \\
\hline MML 1 & $\mathrm{LCC}$ & 7.2 & 0.59 & $0.99 \pm 0.05$ & $1.07 \pm 0.03$ & $4.9 \pm 9.0$ & -1.43 & & & & & \\
\hline MML 8 & LCC & 7.2 & 0.45 & $1.03 \pm 0.05$ & $1.68 \pm 0.03$ & $35.7 \pm 26.0$ & -38.39 & & & $\mathrm{Y}$ & $\mathrm{Y}$ & \\
\hline MML 9 & LCC & 7.2 & 0.48 & $1.04 \pm 0.05$ & $1.05 \pm 0.03$ & $-12.8 \pm 13.8$ & -10.02 & & & $\mathrm{Y}$ & & \\
\hline MML 17 & LCC & 7.2 & 0.35 & $0.99 \pm 0.05$ & $1.60 \pm 0.03$ & $26.9 \pm 11.5$ & -43.54 & & & $\mathrm{Y}$ & $\mathrm{Y}$ & \\
\hline MML 18 & LCC & 7.2 & 0.54 & $0.94 \pm 0.05$ & $0.97 \pm 0.03$ & $-16.9 \pm 26.2$ & -3.09 & & & & & \\
\hline MML 26 & LCC & 7.2 & 0.51 & $0.95 \pm 0.05$ & $0.98 \pm 0.03$ & $-4.0 \pm 20.0$ & -0.09 & & & & & \\
\hline MML 28 & LCC & 7.2 & 0.65 & $1.00 \pm 0.05$ & $1.39 \pm 0.03$ & $37.4 \pm 37.4$ & -43.71 & & & $\mathrm{Y}$ & $\mathrm{Y}$ & \\
\hline MML 32 & $\mathrm{LCC}$ & 7.2 & 0.36 & $0.94 \pm 0.05$ & $1.01 \pm 0.03$ & $16.2 \pm 30.8$ & -0.22 & & & & & \\
\hline MML 36 & UCL & 7.2 & 0.51 & $1.03 \pm 0.05$ & $1.49 \pm 0.03$ & $6.1 \pm 6.5$ & -50.52 & & & $\mathrm{Y}$ & $\mathrm{Y}$ & \\
\hline MML 38 & UCL & 7.2 & 0.52 & $1.02 \pm 0.05$ & $1.04 \pm 0.03$ & $-3.1 \pm 18.6$ & -3.00 & & & $\mathrm{Y}$ & & \\
\hline MML 40 & UCL & 7.2 & 0.50 & $1.03 \pm 0.05$ & $1.00 \pm 0.03$ & $-8.7 \pm 12.2$ & -0.75 & & & & & \\
\hline MML 43 & UCL & 7.2 & 0.46 & $1.02 \pm 0.05$ & $1.12 \pm 0.03$ & $-22.1 \pm 23.4$ & -2.70 & & & $\mathrm{Y}$ & $\mathrm{Y}$ & \\
\hline MML 51 & UCL & 7.2 & 0.64 & $1.00 \pm 0.05$ & $1.00 \pm 0.03$ & $4.5 \pm 8.4$ & -0.17 & & & & & \\
\hline MML 57 & UCL & 7.2 & 0.37 & $1.03 \pm 0.05$ & $1.09 \pm 0.03$ & $22.4 \pm 37.0$ & -9.90 & & & $\mathrm{Y}$ & & \\
\hline PDS 66 & LCC & 7.2 & 0.78 & $8.41 \pm 0.10$ & $32.70 \pm 0.02$ & $265.2 \pm 19.5$ & -106.27 & $\mathrm{Y}$ & Y & $\mathrm{Y}$ & $\mathrm{Y}$ & $\mathrm{Y}$ \\
\hline [PZ99] J155847.8-175800 & USco & 6.7 & 0.65 & $0.96 \pm 0.05$ & $1.45 \pm 0.03$ & $-47.9 \pm 32.6$ & -30.41 & & & $\mathrm{Y}$ & $\mathrm{Y}$ & \\
\hline [PZ99] J160814.7-190833 & USco & 6.7 & 0.56 & $0.93 \pm 0.05$ & $0.98 \pm 0.03$ & $15.6 \pm 32.2$ & -0.22 & & & & & \\
\hline [PZ99] J161318.6-221248 & USco & 6.7 & 0.54 & $0.90 \pm 0.05$ & $1.01 \pm 0.03$ & $-20.8 \pm 12.2$ & -1.30 & & & & & \\
\hline [PZ99] J161329.3-231106 & USco & 6.7 & 0.54 & $0.97 \pm 0.05$ & $1.02 \pm 0.03$ & $29.2 \pm 25.6$ & -0.86 & & & & & \\
\hline [PZ99] J161402.1-230101 & USco & 6.7 & 0.39 & $0.96 \pm 0.05$ & $1.07 \pm 0.03$ & $-52.7 \pm 35.1$ & -3.00 & & & $\mathrm{Y}$ & & \\
\hline [PZ99] J161411.0-230536 & USco & 6.7 & 0.57 & $3.73 \pm 0.05$ & $7.39 \pm 0.02$ & $20.1 \pm 3.0$ & -126.65 & $\mathrm{Y}$ & $\mathrm{Y}$ & $\mathrm{Y}$ & $\mathrm{Y}$ & $\mathrm{Y}$ \\
\hline [PZ99] J161459.2-275023 & USco & 6.7 & 0.49 & $1.04 \pm 0.05$ & $1.58 \pm 0.03$ & $86.3 \pm 81.3$ & -41.39 & & & $\mathrm{Y}$ & $\mathrm{Y}$ & \\
\hline [PZ99] J161618.0-233947 & USco & 6.7 & 0.50 & $1.03 \pm 0.05$ & $1.11 \pm 0.03$ & $-28.6 \pm 27.2$ & -7.04 & & & $\mathrm{Y}$ & $\mathrm{Y}$ & \\
\hline QT And & Field & 7.8 & 0.66 & $0.99 \pm 0.05$ & $1.02 \pm 0.03$ & $-8.6 \pm 6.0$ & -1.72 & & & & & \\
\hline R3 & IC 2602 & 7.7 & 0.51 & $1.04 \pm 0.05$ & $0.96 \pm 0.03$ & $-62.4 \pm 53.6$ & -1.46 & & & & & \\
\hline R45 & IC 2602 & 7.7 & 0.34 & $\cdots$ & $1.11 \pm 0.03$ & $12.6 \pm 117.4$ & $\cdots$ & & & & & \\
\hline R83 & IC 2602 & 7.7 & 0.38 & $0.98 \pm 0.05$ & $0.97 \pm 0.03$ & $-125.6 \pm 65.8$ & -0.51 & & & & & \\
\hline RE J0137+18A & Field & 6.8 & 0.76 & $1.05 \pm 0.05$ & $0.95 \pm 0.02$ & $1.4 \pm 2.3$ & -0.08 & & & & & \\
\hline RE J0723+20 & Field & 8.1 & 0.76 & $1.05 \pm 0.05$ & $1.00 \pm 0.02$ & $0.6 \pm 3.0$ & -0.92 & & & & & \\
\hline RX J0258.4+2947 & Field & 8.0 & 0.51 & $0.89 \pm 0.05$ & $0.98 \pm 0.03$ & $-25.3 \pm 19.2$ & -1.74 & & & & & \\
\hline RX J0329.1+0118 & Field & 7.8 & 0.32 & $0.93 \pm 0.05$ & $0.98 \pm 0.03$ & $11.2 \pm 25.9$ & -1.41 & & & & & \\
\hline RX J0331.1+0713 & Field & 6.4 & 0.65 & $0.89 \pm 0.05$ & $0.97 \pm 0.03$ & $-8.8 \pm 9.8$ & -3.26 & & & & & \\
\hline RX J0354.4+0535 & Field & 8.2 & 0.34 & $0.98 \pm 0.05$ & $1.09 \pm 0.03$ & $-24.0 \pm 16.7$ & -2.40 & & & & & \\
\hline RX J0357.3+1258 & Field & 7.8 & 0.44 & $1.01 \pm 0.05$ & $1.04 \pm 0.03$ & $-14.7 \pm 25.6$ & -1.68 & & & & & \\
\hline RX J0434.3+0226 & Field & 7.8 & 0.70 & $0.95 \pm 0.05$ & $0.94 \pm 0.03$ & $-38.0 \pm 26.3$ & -1.17 & & & & & \\
\hline RX J0442.5+0906 & Field & 7.8 & 0.45 & $0.95 \pm 0.05$ & $0.97 \pm 0.03$ & $6.4 \pm 23.1$ & -0.37 & & & & & \\
\hline RX J0849.2-7735 & Field & 8.2 & 0.60 & $1.03 \pm 0.05$ & $1.03 \pm 0.02$ & $0.7 \pm 1.9$ & -1.04 & & & & & \\
\hline
\end{tabular}


Table 3

(Continued)

\begin{tabular}{|c|c|c|c|c|c|c|c|c|c|c|c|c|}
\hline \multirow[t]{2}{*}{ Name } & \multirow{2}{*}{$\begin{array}{l}\text { Stellar } \\
\text { Group }^{\mathrm{a}}\end{array}$} & \multirow[t]{2}{*}{$\log$ (age/years) } & \multirow{2}{*}{$\begin{array}{c}\left(J-K_{\mathrm{s}}\right)_{\mathrm{o}}{ }^{\mathrm{b}} \\
(\mathrm{mag})\end{array}$} & \multicolumn{3}{|c|}{ Normalized Ratios $^{\mathrm{c}}$} & \multirow[t]{2}{*}{$\log p^{d}$} & \multicolumn{5}{|c|}{ Excess? } \\
\hline & & & & $R_{16 / 8}$ & $R_{24 / 8}$ & $R_{70 / 8}$ & & $8 \mu \mathrm{m}$ & $16 \mu \mathrm{m}$ & IRS & $24 \mu \mathrm{m}$ & $70 \mu \mathrm{m}$ \\
\hline RX J0850.1-7554 & Field & 7.8 & 0.50 & $1.02 \pm 0.05$ & $1.03 \pm 0.03$ & $11.6 \pm 12.4$ & -0.29 & & & & & \\
\hline RX J0853.1-8244 & Field & 8.6 & 0.59 & $0.97 \pm 0.05$ & $1.01 \pm 0.03$ & $-24.7 \pm 15.3$ & -0.23 & & & & & \\
\hline RX J0917.2-7744 & Field & 7.8 & 0.44 & $1.01 \pm 0.05$ & $0.96 \pm 0.03$ & $12.0 \pm 12.5$ & -0.16 & & & & & \\
\hline RX J1111.7-7620 & Field & 6.7 & 1.13 & $3.72 \pm 0.05$ & $9.49 \pm 0.02$ & $84.2 \pm 6.9$ & -103.16 & $\mathrm{Y}$ & $\mathrm{Y}$ & $\mathrm{Y}$ & $\mathrm{Y}$ & $\mathrm{Y}$ \\
\hline RX J1140.3-8321 & Field & 7.8 & 0.56 & $0.87 \pm 0.05$ & $0.95 \pm 0.03$ & $-5.9 \pm 14.8$ & -4.72 & & & & & \\
\hline RX J1203.7-8129 & Field & 8.2 & 0.55 & $1.02 \pm 0.05$ & $1.01 \pm 0.03$ & $12.2 \pm 22.5$ & -0.17 & & & & & \\
\hline RX J1209.8-7344 & Field & 8.4 & 0.56 & $0.91 \pm 0.05$ & $0.99 \pm 0.03$ & $-8.7 \pm 9.9$ & -0.11 & & & & & \\
\hline RX J1220.6-7539 & Field & 7.8 & 0.55 & $0.98 \pm 0.05$ & $0.99 \pm 0.03$ & $-20.9 \pm 7.4$ & -1.39 & & & & & \\
\hline RX J1225.3-7857 & Field & 8.2 & 0.49 & $0.97 \pm 0.05$ & $0.98 \pm 0.03$ & $-2.8 \pm 11.7$ & -0.48 & & & & & \\
\hline RX J1450.4-3507 & UCL & 7.2 & 0.55 & $1.02 \pm 0.05$ & $0.98 \pm 0.03$ & $-7.0 \pm 7.6$ & -0.63 & & & & & \\
\hline RX J1457.3-3613 & UCL & 7.2 & 0.42 & $0.96 \pm 0.05$ & $1.04 \pm 0.03$ & $-2.8 \pm 12.3$ & -1.01 & & & & & \\
\hline RX J1458.6-3541 & UCL & 7.2 & 0.66 & $1.02 \pm 0.05$ & $0.99 \pm 0.03$ & $11.9 \pm 6.7$ & -0.45 & & & & & \\
\hline RX J1500.8-4331 & UCL & 7.2 & 0.53 & $0.92 \pm 0.05$ & $0.99 \pm 0.03$ & $-35.2 \pm 28.9$ & -0.63 & & & & & \\
\hline RX J1507.2-3505 & UCL & 7.2 & 0.51 & $0.97 \pm 0.05$ & $1.06 \pm 0.03$ & $-0.2 \pm 15.1$ & -0.56 & & & & & \\
\hline RX J1518.4-3738 & UCL & 7.2 & 0.51 & $0.94 \pm 0.05$ & $1.02 \pm 0.03$ & $-4.8 \pm 15.9$ & -0.77 & & & & & \\
\hline RX J1531.3-3329 & Field & 8.0 & 0.56 & $0.91 \pm 0.05$ & $0.95 \pm 0.03$ & $44.9 \pm 47.5$ & -2.52 & & & & & \\
\hline RX J1541.1-2656 & USco & 6.7 & 0.47 & $0.96 \pm 0.05$ & $1.00 \pm 0.03$ & $-153.7 \pm 56.1$ & -1.77 & & & & & \\
\hline RX J1544.0-3311 & UCL & 7.2 & 0.58 & $\ldots$ & $1.04 \pm 0.03$ & $-54.1 \pm 29.3$ & $\ldots$ & & & & & \\
\hline RX J1545.9-4222 & UCL & 7.2 & 0.64 & $0.92 \pm 0.05$ & $1.01 \pm 0.03$ & $-44.0 \pm 20.1$ & -0.40 & & & & & \\
\hline RX J1600.6-2159 & USco & 6.7 & 0.52 & $0.94 \pm 0.05$ & $1.15 \pm 0.03$ & $24.5 \pm 38.9$ & -10.02 & & & $\mathrm{Y}$ & $\mathrm{Y}$ & \\
\hline RX J1839.0-3726 & $\mathrm{CrA}$ & 7.2 & 0.49 & $0.94 \pm 0.05$ & $1.08 \pm 0.03$ & $-23.0 \pm 21.7$ & -0.99 & & & & & \\
\hline RX J1841.8-3525 & $\mathrm{CrA}$ & 7.2 & 0.50 & $0.98 \pm 0.05$ & $1.03 \pm 0.03$ & $-3.0 \pm 9.7$ & -1.21 & & & & & \\
\hline RX J1842.9-3532 & $\mathrm{CrA}$ & 6.6 & 1.16 & $3.95 \pm 0.05$ & $17.94 \pm 0.02$ & $429.3 \pm 32.0$ & -71.22 & $\mathrm{Y}$ & $\mathrm{Y}$ & $\mathrm{Y}$ & $\mathrm{Y}$ & $\mathrm{Y}$ \\
\hline RX J1844.3-3541 & $\mathrm{CrA}$ & 6.2 & 0.67 & $1.01 \pm 0.05$ & $0.97 \pm 0.03$ & $-42.8 \pm 15.9$ & -2.30 & & & & & \\
\hline RX J1852.3-3700 & $\mathrm{CrA}$ & 6.8 & 0.61 & $11.09 \pm 0.34$ & $124.31 \pm 0.03$ & $3281.9 \pm 259.6$ & -116.17 & $\mathrm{Y}$ & $\mathrm{Y}$ & $\mathrm{Y}$ & $\mathrm{Y}$ & $\mathrm{Y}$ \\
\hline RX J1917.4-3756 & $\mathrm{CrA}$ & 6.8 & 0.67 & $0.95 \pm 0.05$ & $1.02 \pm 0.03$ & $2.2 \pm 7.2$ & -1.10 & & & & & \\
\hline $\mathrm{RX} \mathrm{J} 2313.0+2345$ & Field & 7.0 & 0.33 & $1.02 \pm 0.05$ & $0.96 \pm 0.03$ & $-10.7 \pm 14.1$ & -1.28 & & & & & \\
\hline SAO 150676 & Field & 7.8 & 0.37 & $1.01 \pm 0.05$ & $1.06 \pm 0.03$ & $2.4 \pm 4.8$ & -9.57 & & & $\mathrm{Y}$ & & \\
\hline SAO 178272 & Field & 8.0 & 0.64 & $1.00 \pm 0.05$ & $1.02 \pm 0.03$ & $1.6 \pm 3.7$ & -3.67 & & & $\mathrm{Y}$ & & \\
\hline ScoPMS 21 & USco & 6.7 & 0.64 & $0.93 \pm 0.05$ & $1.04 \pm 0.03$ & $-21.9 \pm 70.2$ & -2.52 & & & & & \\
\hline ScoPMS 27 & USco & 6.7 & 0.66 & $0.94 \pm 0.05$ & $1.01 \pm 0.03$ & $12.8 \pm 20.0$ & -0.55 & & & & & \\
\hline ScoPMS 52 & USco & 6.7 & 0.57 & $\ldots$ & $0.99 \pm 0.03$ & $34.9 \pm 24.1$ & $\ldots$ & & & & & \\
\hline ScoPMS 214 & USco & 6.7 & 0.64 & $1.07 \pm 0.05$ & $1.17 \pm 0.03$ & $20.6 \pm 21.1$ & -16.41 & & & $\mathrm{Y}$ & $\mathrm{Y}$ & \\
\hline V343 Nor & Field & 7.1 & 0.53 & $1.00 \pm 0.05$ & $1.06 \pm 0.02$ & $-0.3 \pm 10.6$ & -0.16 & & & & & \\
\hline V383 Lac & Field & 7.8 & 0.53 & $0.99 \pm 0.05$ & $1.06 \pm 0.02$ & $3.8 \pm 4.2$ & -0.32 & & & & & \\
\hline vB 1 & Hyades & 8.8 & 0.30 & $1.01 \pm 0.05$ & $0.98 \pm 0.02$ & $4.4 \pm 3.8$ & -0.71 & & & & & \\
\hline vB 39 & Hyades & 8.8 & 0.37 & $1.02 \pm 0.05$ & $1.02 \pm 0.02$ & $-1.4 \pm 4.0$ & -0.53 & & & & & \\
\hline vB 49 & Hyades & 8.8 & 0.28 & $1.00 \pm 0.05$ & $0.99 \pm 0.02$ & $-3.9 \pm 5.1$ & -0.09 & & & & & \\
\hline vB 52 & Hyades & 8.8 & 0.36 & $1.02 \pm 0.05$ & $1.01 \pm 0.02$ & $0.3 \pm 4.4$ & -0.08 & & & & & \\
\hline vB 63 & Hyades & 8.8 & 0.41 & $1.01 \pm 0.05$ & $1.01 \pm 0.02$ & $1.4 \pm 5.0$ & -0.52 & & & & & \\
\hline vB 64 & Hyades & 8.8 & 0.35 & $0.99 \pm 0.05$ & $1.00 \pm 0.02$ & $-2.4 \pm 6.4$ & -0.53 & & & & & \\
\hline vB 66 & Hyades & 8.8 & 0.27 & $1.04 \pm 0.05$ & $1.01 \pm 0.02$ & $3.9 \pm 4.3$ & -0.42 & & & & & \\
\hline vB 73 & Hyades & 8.8 & 0.29 & $1.01 \pm 0.05$ & $1.02 \pm 0.02$ & $-3.9 \pm 4.9$ & -0.91 & & & & & \\
\hline vB 79 & Hyades & 8.8 & 0.47 & $0.73 \pm 0.91$ & $0.99 \pm 0.03$ & $-6.4 \pm 6.6$ & -0.32 & & & & & \\
\hline vB 88 & Hyades & 8.8 & 0.28 & $1.02 \pm 0.05$ & $1.02 \pm 0.02$ & $-5.6 \pm 6.2$ & -0.66 & & & & & \\
\hline vB 91 & Hyades & 8.8 & 0.52 & $1.00 \pm 0.05$ & $1.01 \pm 0.02$ & $-5.9 \pm 8.0$ & -0.07 & & & & & \\
\hline vB 92 & Hyades & 8.8 & 0.38 & $0.98 \pm 0.05$ & $0.99 \pm 0.02$ & $-1.9 \pm 10.4$ & -0.07 & & & & & \\
\hline vB 93 & Hyades & 8.8 & 0.49 & $1.00 \pm 0.05$ & $0.99 \pm 0.03$ & $8.2 \pm 9.1$ & -0.94 & & & & & \\
\hline vB 96 & Hyades & 8.8 & 0.52 & $0.99 \pm 0.05$ & $0.99 \pm 0.02$ & $-6.7 \pm 6.1$ & -0.04 & & & & & \\
\hline vB 97 & Hyades & 8.8 & 0.30 & $1.01 \pm 0.05$ & $1.01 \pm 0.02$ & $-2.6 \pm 5.8$ & -0.96 & & & & & \\
\hline vB 99 & Hyades & 8.8 & 0.49 & $0.99 \pm 0.05$ & $1.00 \pm 0.03$ & $-5.3 \pm 13.7$ & -0.79 & & & & & \\
\hline vB 106 & Hyades & 8.8 & 0.37 & $1.02 \pm 0.05$ & $1.01 \pm 0.02$ & $4.9 \pm 5.5$ & -1.15 & & & & & \\
\hline vB 142 & Hyades & 8.8 & $\ldots$ & $1.02 \pm 0.05$ & $0.99 \pm 0.02$ & $1.4 \pm 7.6$ & -1.48 & & & & & \\
\hline vB 143 & Hyades & 8.8 & 0.26 & $0.99 \pm 0.05$ & $1.00 \pm 0.02$ & $-2.8 \pm 6.6$ & -0.86 & & & & & \\
\hline vB 176 & Hyades & 8.8 & 0.55 & $1.03 \pm 0.05$ & $0.99 \pm 0.02$ & $-0.4 \pm 4.6$ & -3.17 & & & & & \\
\hline vB 180 & Hyades & 8.8 & 0.48 & $1.06 \pm 0.05$ & $1.00 \pm 0.03$ & $2.1 \pm 6.3$ & -0.38 & & & & & \\
\hline vB 183 & Hyades & 8.8 & 0.51 & $0.98 \pm 0.05$ & $1.00 \pm 0.03$ & $-15.3 \pm 16.3$ & -3.04 & & & & & \\
\hline W79 & IC 2602 & 7.7 & 0.46 & $1.04 \pm 0.05$ & $1.22 \pm 0.03$ & $1.4 \pm 114.5$ & -1.24 & & & & & \\
\hline
\end{tabular}

Notes.

a $\alpha$ Per: Alpha Perseus; CrA: Corona Australis; LCC: Lower Centaurus Crux; UCL: Upper Centaurus Crux; USco: Upper Scorpius.

${ }^{\mathrm{b}}$ Dereddened $J-K_{S}$ color.

${ }^{c}$ Ratio of the observed flux density ratio to the photospheric value.

${ }^{\mathrm{d}}$ Probability statistic computed from $F$-test for fitting model photospheres and modified blackbodies to IRS spectra. 
photosphere for most stars in our sample. This expectation is confirmed from inspection of a $J-H$ versus $H-K_{\mathrm{s}}$ diagram which shows that only two stars (RX J1111.7-7620 and RX J1842.9-3532) exhibit a $K_{\mathrm{s}}$-band excess detectable by this technique (see, e.g., Meyer et al. 1997, for a discussion of the merits and limitations of this diagram). In Figure 1, most sources lie along a tight locus of points, while five sources (RX J1111.7-7620, RX J1842.9-3532, RX J1852.3-3700, PDS 66, [PZ99] J161411.0-230536) have values of $R_{8 / 3.6}$ well above the locus and have a clear $8 \mu \mathrm{m}$ excess. Four of these sources have ages $<10 \mathrm{Myr}$, and the fifth (PDS 66) is a member of the Lower Centaurus Crux association with an age of $\sim 12 \mathrm{Myr}$ (Preibisch \& Mamajek 2008). Silverstone et al. (2006) showed that these five sources have infrared excesses over a broad range of wavelengths and other properties characteristic of optically thick, circumstellar accretion disks.

In the bottom panel of Figure 1, we present the same diagram after removing the five sources with strong $8 \mu \mathrm{m}$ excesses to emphasize the colors for the majority of stars. The dashed line indicates the best-fit linear relation to the trend between $J-K_{\mathrm{s}}$ color and $\log R_{8 / 3.6}$. We assume that the trend represents intrinsic variation in the photospheric value of $R_{8 / 3.6}$ over the spectral-type range in the FEPS sample. The dispersion about the best-fit line is $\sigma\left(\log R_{8 / 3.6}\right)=0.0043$, or $\sigma\left(R_{8 / 3.6}\right) / R_{8 / 3.6}=$ $1.0 \%$, while the expected dispersion from the observational uncertainties is 0.0044 . The maximum outlier with a positive apparent excess is $3.3 \sigma$ (HD 77407), and we expect $\sim 1$ outlier more than $3 \sigma$ from the mean based on the sample size. The IRS spectrum for HD 77407 shows no evidence for an $8 \mu \mathrm{m}$ excess (Section 3.2), and this source was one of two objects where the IRAC photometry was contaminated by a latent image (see Paper I). We conclude that outside of the five sources with strong $8 \mu \mathrm{m}$ excesses characteristic of optically thick disks, no individual source shows conclusive evidence of a weak $(3 \sigma$ limit of $3 \%$ above the photosphere) $8 \mu \mathrm{m}$ excess indicative of optically thin dust.

\subsection{IRS Low-Resolution Spectra}

To quantify the presence of an infrared excess in the IRS spectra, we determined if the observed spectra are better fitted by a model photosphere alone, or by the sum of a model photosphere and a modified blackbody that represents circumstellar dust emission. The photospheric component was derived by fitting Kurucz synthetic spectra to optical and nearinfrared photometry between 0.5 and $2.2 \mu \mathrm{m}$. The stellar effective temperature and visual extinction were free parameters in the fits, while the surface gravity and metallicity were fixed (see Paper I for details of the fitting procedure).

These model spectra cannot be compared directly with the IRS spectra to infer the presence of an infrared excess for two reasons. First, the mean flux density of the model often differs from the observed spectra, which may reflect either uncertainties in the model fit or calibration uncertainties in the observed spectrum. Second, in some spectra an offset is present between the SL1 and LL2 IRS orders that is manifested as an abrupt jump in the flux density at a wavelength of $14.2 \mu \mathrm{m}$. To correct for these offsets, the best-fit Kurucz model to the broadband photometry was renormalized to the IRS spectrum. The renormalization included a term to account for an overall flux density offset between the model and the observed spectrum, and a second term to account for the offset between the SL1 and LL2 orders.
The variance between the Kurucz model fit (including the flux offset terms) and the observed IRS spectra was computed between 12 and $35 \mu \mathrm{m}$. The same IRS spectrum was then fitted with a Kurucz model plus modified blackbody [i.e., $\left.S_{v} \propto v^{3+\beta} /\left(e^{h v /\left(k T_{\mathrm{d}}\right)}-1\right)\right]$ that represents thermal emission from dust grains. The free parameters for the modified blackbody are the dust temperature $\left(T_{\mathrm{d}}\right)$ and the solid angle of dust grains, which is proportional to the total cross-sectional surface area if the grains are at a single temperature. The appropriate value of $\beta$ depends on the grain properties that contribute emission in the IRS wavelengths. In practice, the IRS spectra probe the Wien tail of the dust emission (see discussion below) and do not place meaningful constraints on $\beta$. Therefore, we assume $\beta=0.8$ to conform with the typical value inferred from submillimeter observations of debris disks (Williams \& Andrews 2006). Adopting a blackbody function $(\beta=0)$ produces warmer dust temperatures but does not otherwise alter the results of our analysis. The variance from the Kurucz spectrum fit alone and that from the Kurucz spectra plus modified blackbody were compared by computing the $F$-test statistic ( $\equiv p$; Press et al. 2002). If $p \ll 1$, we conclude that the Kurucz spectrum alone is a poor fit to the IRS data.

Protassov et al. (2002) emphasized that the probability distribution from the $F$-test is not formally valid for this application since the second model adds a modified blackbody component that is not present in the first model. We conducted Monte Carlo simulations to establish the correct probability distribution where we took the best-fit Kurucz model spectrum, introduced a random offset to the SL1 order, and added wavelength-dependent random noise to the spectrum. We then repeated the $F$-test analysis for this synthetic spectrum. The empirical probability distribution was derived from 8000 simulated spectra. Simulations were run using the noise characteristics of a relatively faint star (MML $32, \mathrm{~S} / \mathrm{N}$ of 4 in the $32 \mu \mathrm{m}$ bandpass), and a bright star (vB $1, \mathrm{~S} / \mathrm{N}$ of 13). Whereas we expected 80 of the 8000 simulated spectra to have a probability $\leqslant 0.01$ from random noise, the $F$-test yielded 76 for the MML 32 simulation and 86 for vB 1 . Similarly, we expect eight source to have a probability $\leqslant 0.001$, and the simulation yielded 7 and 10 for MML 32 and $\mathrm{vB} 1$, respectively. We conclude that the $F$-test, while not formally valid for this application, nonetheless provides a reasonably accurate probability distribution.

In Figure 2, we present IRS low-resolution spectra for four sources to illustrate the fitting results. For the star HIP 76477, we derived $p=0.4$ and conclude that the IRS spectrum is consistent with photospheric emission. The spectrum indeed shows that the $S_{v} v^{-2}$ spectrum is roughly constant versus wavelength, which is expected for these sources in the absence of dust since the emission is approximately in the Rayleigh-Jeans limit at these wavelengths. The other three sources in Figure 2 have $p<10^{-3}$, which indicates that the spectra are poorly represented by Kurucz models. For these sources, the observed emission systematically exceeds a constant $S_{v} v^{-2}$ spectrum at the longer IRS wavelengths and is consistent with the presence of emission from dust grains.

In principle, we can select a reliable list of sources with probable IRS excesses based solely on the $F$-test statistic. In practice, several sources have a low spectral intensity at long wavelengths $(\gtrsim 30 \mu \mathrm{m})$ relative to the Kurucz model and are parameterized by a negative solid angle in the modified blackbody fits. These spectra clearly do not result from dust emission, and likely indicate errors in the spectral extraction or low $\mathrm{S} / \mathrm{N}$ in the IRS spectra at the longer wavelengths. 


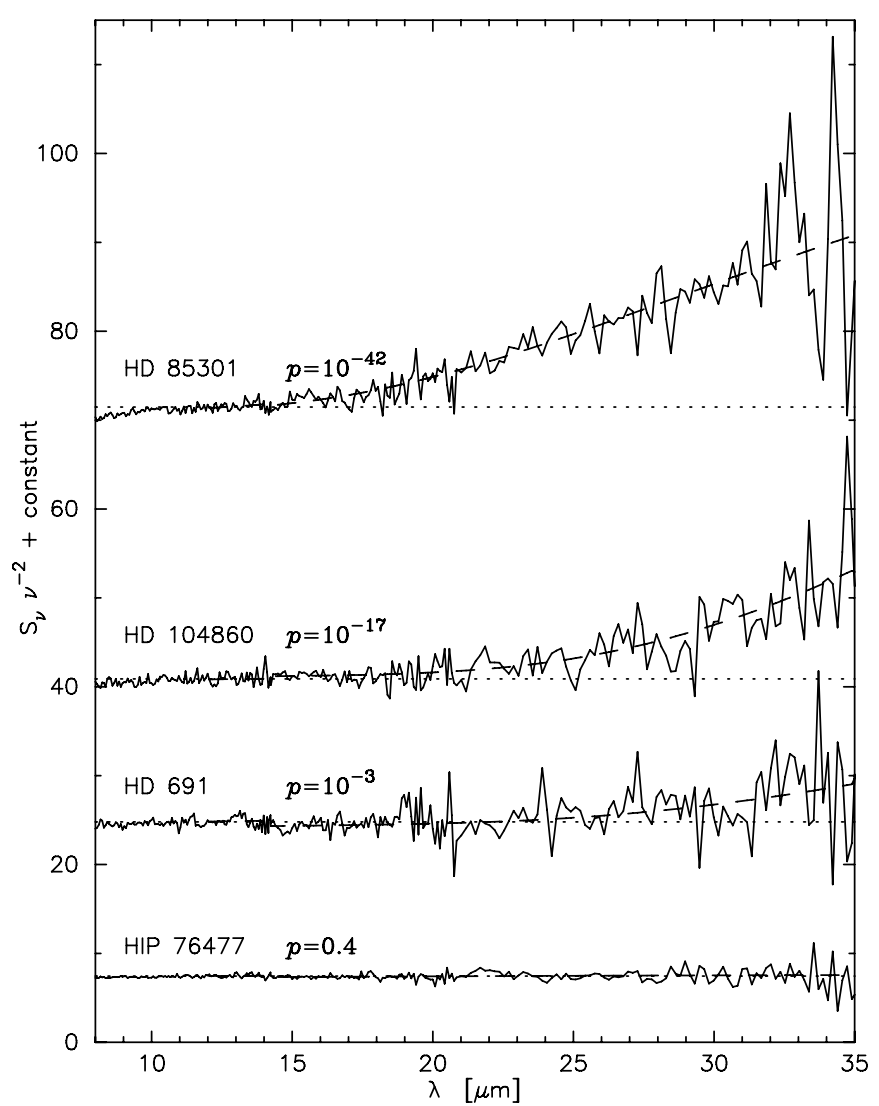

Figure 2. Observed IRS low-resolution spectra for four stars in the FEPS sample to illustrate how infrared excesses in the spectra were identified. The dotted line indicates the average value of $S_{v} v^{-2}$ between 12 and $14 \mu \mathrm{m}$, and the dashed curve is the best-fit Kurucz model (including flux offset terms; see text) plus modified blackbody. The value of $p$ is the $F$-test statistic that compares the variance from a Kurucz-model only fit to the IRS spectra and a Kurucz-model plus modified blackbody. The lower the value of $p$, the less likely the Kurucz model alone is a good fit to the IRS spectrum. HIP 76477 is an example of a star where the IRS spectrum is consistent with a stellar photosphere. The other three sources have emission that departs from the stellar photosphere at longer wavelengths.

We imposed the following criteria then to select sources with candidate IRS excesses: (1) the $\mathrm{S} / \mathrm{N}$ in a synthetic bandpass between 30 and $34 \mu \mathrm{m}$ is $\geqslant 3$; (2) the probability from the $F$-test is $p \leqslant 0.003$, and (3) the solid angle of dust emission is $>0$. In total, 71 sources satisfied these criteria. However, whereas we expected one source detected at $32 \mu \mathrm{m}$ to have such negative solid angles for $p \leqslant 0.003$, the analysis yielded seven such sources. In Section 3.6, we combine the IRS and MIPS data to select a reliable sample of infrared excess sources that exhibit infrared excesses in both instruments.

From visual inspection of the IRS spectra, the star 1RXS J051111.1+281353 had a spectral shape that was not amenable to the above analysis. In Figure 3, we present the spectral energy distribution between 5 and $35 \mu \mathrm{m}$ for this star, including the IRS spectrum (solid curve), IRAC and MIPS photometry (filled circles), and a Kurucz synthetic spectrum (dashed curve) normalized to optical and near-infrared photometry (see Paper I). The IRS spectrum shows an apparent excess above the stellar photosphere between 9 and $28 \mu \mathrm{m}$, with perhaps a 10 $\mu \mathrm{m}$ silicate emission feature. The shape of the excess emission is distinct from the other FEPS sources where the excess emission increases toward longer wavelengths, and suggests that the excess emission in 1RXS J051111.1+281353

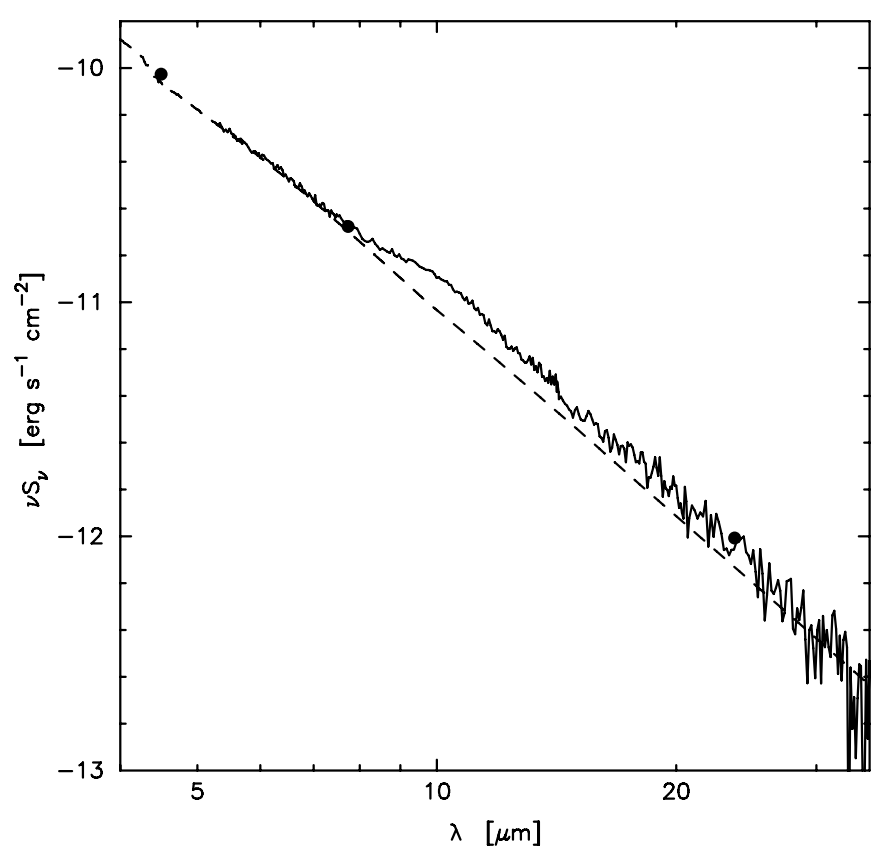

Figure 3. Spectral energy distribution for 1RXS J051111.1+281353 between 5 and $35 \mu \mathrm{m}$. The solid curve is the IRS low-resolution spectrum, the dashed curved is the Kurucz synthetic spectrum normalized to optical and near-infrared photometry (see Paper I), and the solid circles represent IRAC and MIPS broadband photometry. Comparison of the IRS and model spectra suggests the presence of an infrared excess between 8 and $28 \mu \mathrm{m}$.

originates primarily from warm dust grains. The star HD 72905 shows similar characteristics (Beichman et al. 2006b). The excess was not revealed by fitting a modified blackbody to the IRS spectrum since this analysis allowed for an normalization constant, which removed the low-level infrared excess. A more detailed analysis of the spectrum is forthcoming. In the following analysis, we include 1RXS J051111.1+281353 as an IRS-verified excess to provide a total of 72 stars with candidate IRS excesses.

\subsection{IRS $16 \mu \mathrm{m}$}

While analysis of the IRS spectra identified sources with infrared excesses between 12 and $35 \mu \mathrm{m}$, in later sections it will be useful to quantify the infrared excess at an intermediate wavelength between the IRAC $8 \mu \mathrm{m}$ and MIPS $24 \mu \mathrm{m}$ bandpasses. For this exercise, we used the $16 \mu \mathrm{m}$ fluxes computed from the IRS spectra (see Section 2). Since the $8 \mu \mathrm{m}$ flux density is mostly photospheric in origin (Section 3.1), we use the 16 to $8 \mu \mathrm{m}$ flux density ratio $\left(\equiv R_{16 / 8}\right)$ to identify any stars with $16 \mu$ m excesses. After excluding the five known optically thick disks with IRAC excesses, the median value of $R_{16 / 8}$ for the FEPS observations is 0.244 with a dispersion about the median of $\sigma\left(R_{16 / 8}\right)=5.4 \%$, which we adopt as the photospheric value and minimum uncertainty in $R_{16 / 8}$, respectively. Of the 314 sources in the sample, only the five stars that are surrounded by optically thick disks have a $16 \mu \mathrm{m}$ excess more than $3 \sigma\left(R_{16 / 8}\right)=16.2 \%$ above the photosphere. The star 1RXS J051111.1+281353 also has a $16 \mu \mathrm{m}$ excess (see Figure 3), but at a lower level.

\subsection{MIPS $24 \mu \mathrm{m}$}

The methods adopted in the literature to identify $24 \mu \mathrm{m}$ excesses include measuring how much the observed $24 \mu \mathrm{m}$ flux density exceeds a model stellar spectrum normalized at optical and near-infrared wavelengths (e.g., Bryden et al. 2006), 


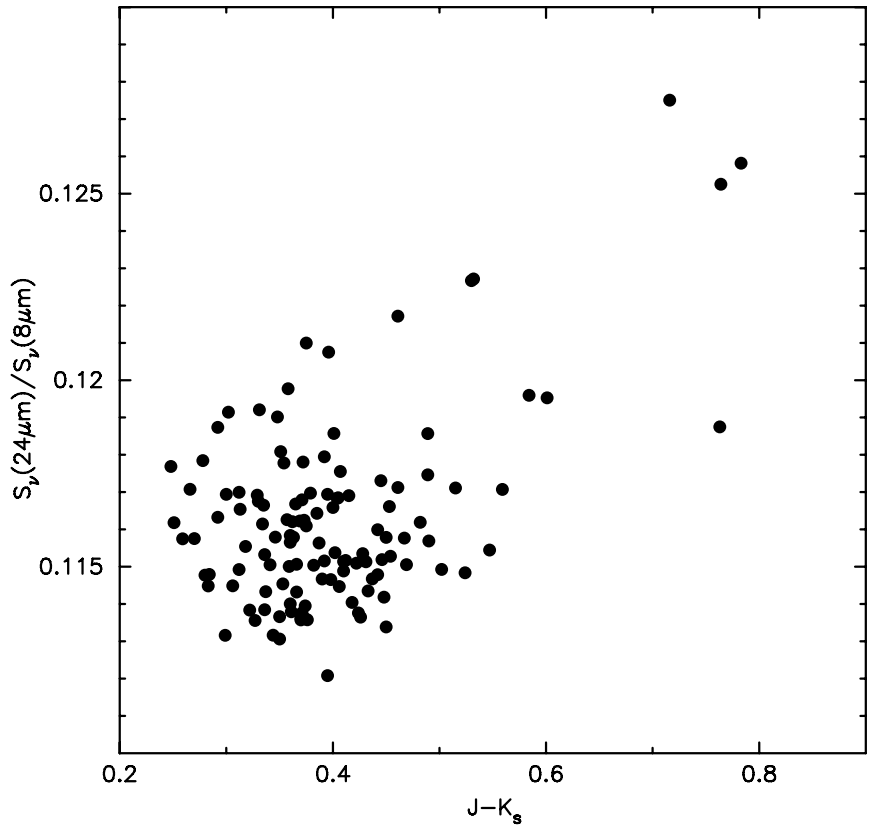

Figure 4. $24 \mu \mathrm{m}$ to $8 \mu \mathrm{m}$ flux density ratio $\left(R_{24 / 8}\right)$ as a function of $J-K_{\mathrm{S}}$ color for stars with $S_{\nu}(8 \mu \mathrm{m}) \geqslant 100 \mathrm{mJy}$ that do not have an IRS excess $(p>0.003)$. An apparent trend exists in that stars with the reddest $J-K_{\mathrm{S}}$ colors tend to have a large value of $R_{24 / 8}$. Photometry was dereddened using the extinction values listed in Paper I.

and identifying sources with anomalously red $K-$ [24] colors (e.g., Siegler et al. 2007). After considering these approaches, we used the 24 to $8 \mu \mathrm{m}$ flux density ratio $\left(\equiv R_{24 / 8}\right)$ which empirically resulted in the most sensitive search for sources with $24 \mu \mathrm{m}$ excesses. Meyer et al. (2008) adopted a similar approach to identify $24 \mu \mathrm{m}$ excesses in the FEPS sample, but used a single detection threshold over all brightness levels to identify excess sources. We extend their analysis by using the final FEPS data processing (see Paper I) and adopting separate detection thresholds for bright and faint sources.

In Figure 4, we show the dependence of $R_{24 / 8}$ on the dereddened Two Micron All Sky Survey (2MASS) $J-K_{\mathrm{S}}$ color, a proxy for spectral type, for stars brighter than $S_{v}(8 \mu \mathrm{m})=100$ mJy that do not have an apparent IRS excess $(p>0.003)$. These criteria were adopted to select high signal-to-noise photometry consistent with photospheric emission. An apparent trend exists in that stars with the largest value of $R_{24 / 8}$ also have red $J-K_{\mathrm{s}}$ colors. All four stars with $J-K_{\mathrm{s}}>0.7$ have spectral types between $\mathrm{K} 0$ and $\mathrm{K} 5$, consistent with an increasing value of $R_{24 / 8}$ toward later spectral types. A similar trend is observed in $K$-band where the $K-$ [24] color is $\sim 0.0 \mathrm{mag}$ over the spectral-type range $\mathrm{F} 2$ to $\mathrm{K} 4$, and becomes redder toward later spectral types (Beichman et al. 2006a). However, we cannot confidently derive the functional dependence on $R_{24 / 8}$ on $J-K_{\mathrm{s}}$ since the trend depends entirely on the four reddest stars. For simplicity, we assume a photospheric value of $R_{24 / 8}=0.116$ for $J-K<0.7$, and $R_{24 / 8}=0.125$ for redder stars, which are the median $R_{24 / 8}$ colors in the respective $J-K_{\mathrm{s}}$ color range of stars without IRS excesses.

In Figure 5, we show the observed $R_{24 / 8}$ ratio, normalized by the adopted photospheric value, as a function of the $8 \mu \mathrm{m}$ flux density. Since the $8 \mu \mathrm{m}$ emission is mostly photospheric in origin (see Section 3.1), sources with a large value of $R_{24 / 8}$ are candidate $24 \mu \mathrm{m}$ excess sources. To determine the minimum detectable $24 \mu \mathrm{m}$ excess, we consider the empirical scatter

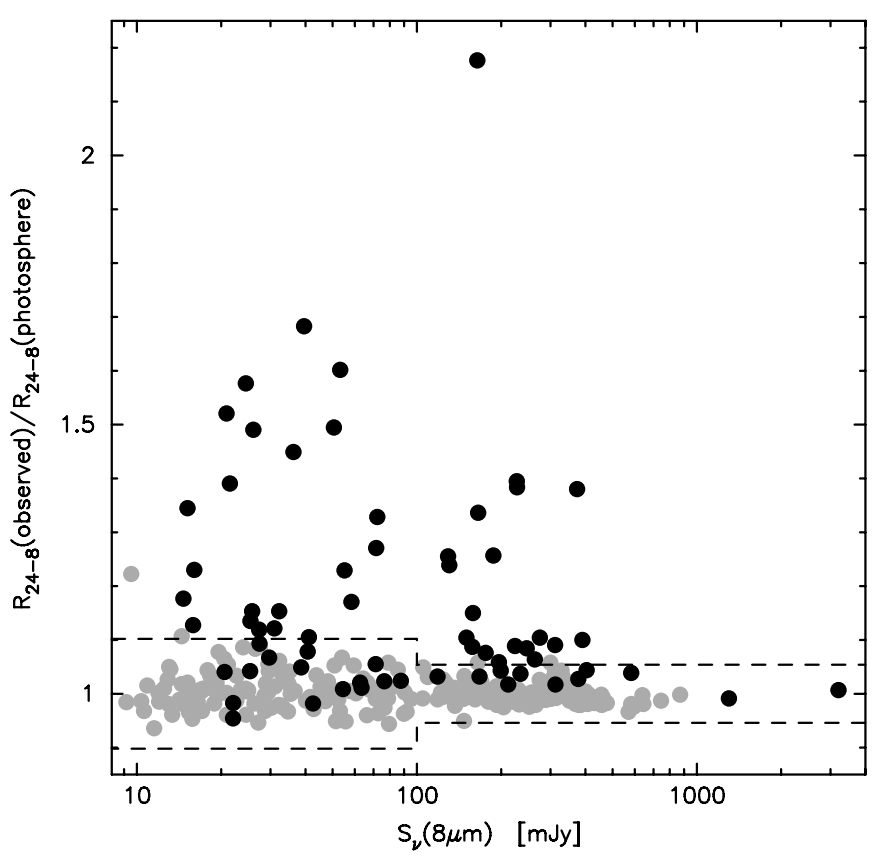

Figure 5. Observed $24 \mu \mathrm{m}$ to $8 \mu \mathrm{m}$ flux density ratio normalized by the photospheric value as a function of the observed $8 \mu \mathrm{m}$ flux density. The dashed lines show the $3 \sigma$ limits used to identify sources with MIPS $24 \mu \mathrm{m}$ excesses, which is a $10.2 \%$ excess for stars fainter than $S_{\nu}(8 \mu \mathrm{m})=100 \mathrm{mJy}$ and $5.4 \%$ for brighter stars. The black circles represent sources that exhibit an apparent excess in the IRS spectra independent of the MIPS $24 \mu \mathrm{m}$ photometry. Five sources with optically thick disks (see Figure 1) are offscale on this plot.

in $R_{24 / 8}$ for sources without an IRS excess $(p>0.003)$ as shown by the gray circles in Figure 5 . The value of the normalized $R_{24 / 8}$ appears nearly constant at a mean value of 1.0 , but the rms about the mean increases for sources fainter than $S_{v}(8 \mu \mathrm{m}) \sim 100 \mathrm{mJy}$. The computed rms after rejecting one outlier point with normalized $R_{24 / 8}>1.2$ (W79; see Section $3.6)$ is $1.8 \%$ and $3.4 \%$ for sources brighter and fainter than $100 \mathrm{mJy}$, respectively. By comparison, the median uncertainty in $R_{24 / 8}$ for the two brightness ranges is $1.1 \%$ and $1.3 \%$, which is smaller than the observed scatter in $R_{24 / 8}$. We adopt a minimum uncertainty in $R_{24 / 8}$ of $3.4 \%$ for stars fainter than $S_{v}(8 \mu \mathrm{m})=100 \mathrm{mJy}$ and $1.8 \%$ for brighter sources. These minimum uncertainties imply a $3 \sigma$ detection limit for a $24 \mu \mathrm{m}$ excess above the photosphere of $10.2 \%$ for faint stars and $5.4 \%$ for bright sources. The detection limits for a $24 \mu \mathrm{m}$ excess are indicated by the dashed lines in Figure 5 . Of the 314 stars in the sample, 50 have a value of $R_{24 / 8}$ more than $3 \sigma$ above the photosphere.

\subsection{MIPS $70 \mu \mathrm{m}$}

Photometric excesses in the MIPS $70 \mu \mathrm{m}$ band were identified from the comparison of the measured flux densities with the expected photospheric contribution. The $70 \mu \mathrm{m}$ photospheric flux density was estimated from the photospheric value of $R_{24 / 8}$ described in Section 3.4, and further assuming that the intrinsic photospheric [24] - [70] color is 0 mag. We adopt flux densities for a zero magnitude star of 7.14 Jy and $0.775 \mathrm{Jy}$ for the 24 $\mu \mathrm{m}$ and $70 \mu \mathrm{m}$ band respectively as reported on the MIPS calibration Web pages ${ }^{13}$ as of 2007 April 30. Hillenbrand et al. (2008) also identified sources with $70 \mu \mathrm{m}$ excesses in the FEPS data, but they used the Kurucz synthetic spectra normalized to

\footnotetext{
13 http://ssc.spitzer.caltech.edu/mips/calib/.
} 


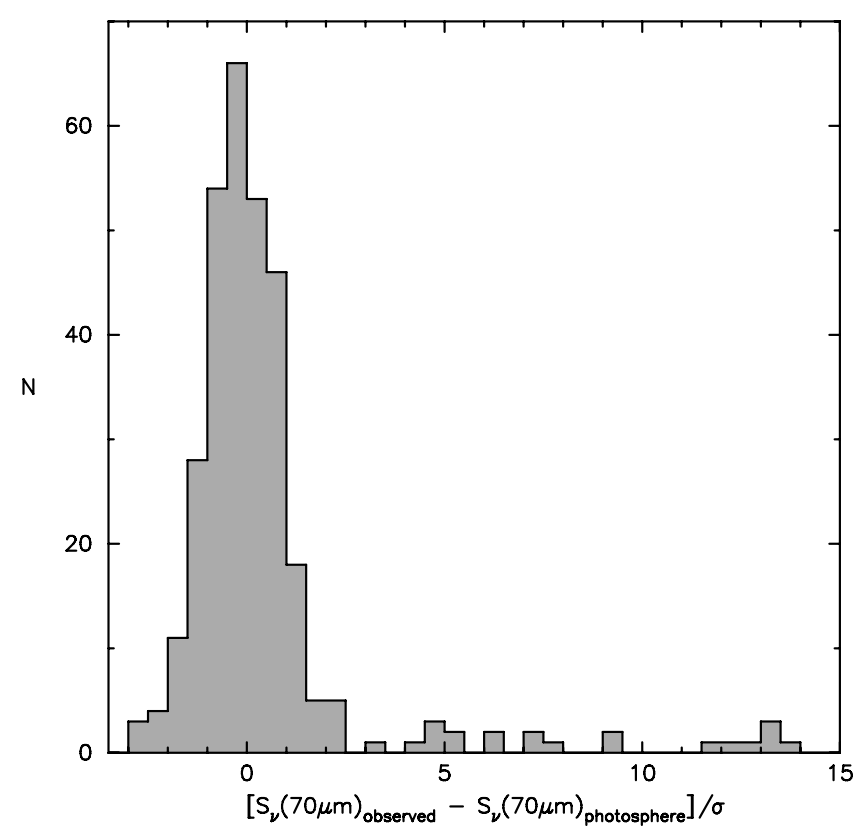

Figure 6. Histogram of the $\mathrm{S} / \mathrm{N}$ of the observed $70 \mu \mathrm{m}$ flux density above the expected stellar photospheric value. The photospheric contribution was estimated from the observed $8 \mu \mathrm{m}$ flux density and an assumed $8 \mu \mathrm{m}$ to $70 \mu \mathrm{m}$ flux density ratio.

optical and near-infrared broad-band photometry to estimate the $70 \mu \mathrm{m}$ photospheric emission.

Of the 314 sources, $70 \mu \mathrm{m}$ emission was detected toward 22 sources with an $\mathrm{S} / \mathrm{N} \geqslant 3$. To establish which sources exhibit a $70 \mu \mathrm{m}$ excess from circumstellar dust, we present in Figure 6 a histogram of the $\mathrm{S} / \mathrm{N}$ of the observed $70 \mu \mathrm{m}$ emission above the stellar photosphere. The $\mathrm{S} / \mathrm{N}$ of the excess is defined as $\left(S_{v}(70 \mu \mathrm{m})_{\text {observed }}-S_{v}(70 \mu \mathrm{m})_{\text {photosphere }}\right) / \sigma$, where $\sigma$ is the uncertainty in the difference between the observed and photospheric flux densities including calibration uncertainties. The $\mathrm{S} / \mathrm{N}$ is centered near zero with a tail toward positive values that may indicate $70 \mu \mathrm{m}$ excess sources. Of the 22 sources detected at $70 \mu \mathrm{m}$, one source (HD 13974) has $70 \mu \mathrm{m}$ emission consistent with the photosphere, and 21 sources have a $70 \mu \mathrm{m}$ excess above the stellar photosphere with an $\mathrm{S} / \mathrm{N}$ greater than 3 . All 21 sources were also identified as $70 \mu \mathrm{m}$ excess sources by Hillenbrand et al. (2008).

\subsection{Synthesis}

We now combine results from the individual instruments to select a list of sources with infrared excesses that will be analyzed in the remainder of this paper. Since the MIPS $24 \mu \mathrm{m}$ bandpass is encompassed by the IRS spectral coverage, any $24 \mu \mathrm{m}$ photometric excess should be verifiable with IRS assuming comparable sensitivity. As discussed in Section 3.2, approximately seven of the candidate IRS sources could be spurious. The expected false detection rate for the MIPS $24 \mu \mathrm{m}$ excesses is $\lesssim 1$ since no sources have observed $R_{24 / 8}$ values more than $3 \sigma$ below the adopted photospheric value (see Figure 5). Given the detection rate of $3 \sigma$ MIPS $24 \mu \mathrm{m}$ excesses $(16 \%)$, the expected number of sources with a valid MIPS $24 \mu \mathrm{m}$ and a false IRS excesses is $\sim 1$. The expected number of sources with a false MIPS $24 \mu \mathrm{m}$ and false IRS excess is $\sim 0.02$. This is consistent with the fact that none of the seven IRS sources with negative solid angles have a $24 \mu \mathrm{m}$ photometric excess. By requiring both an IRS and MIPS
$24 \mu \mathrm{m}$ excess, we aim to create a more reliable sample of IR excesses.

In Figure 5, the 72 sources with apparent IRS excesses (see Section 3.2) are marked as black circles on the colorflux diagram used to identify MIPS $24 \mu \mathrm{m}$ excesses. Of the 50 sources with $\mathrm{a} \geqslant 3 \sigma$ photometric excess at $24 \mu \mathrm{m}, 46$ are also identified with an IRS excess. Conversely, 26 sources have an IRS excess but not a $24 \mu \mathrm{m}$ photometric excess.

The four sources with MIPS $24 \mu \mathrm{m}$ excesses that are not verified spectroscopically are R45, V343 Nor, and V383 Lac, and W79. R45 has a $10.2 \%$ excess with an $\mathrm{S} / \mathrm{N}$ of 3.0 and is at the limit to define a $24 \mu \mathrm{m}$ excess. R45 is located in bright nebulosity and the extracted IRS spectrum has negative flux densities at the longer wavelengths, perhaps because of poor background subtraction. The apparent $24 \mu \mathrm{m}$ photometric excesses in V343 Nor and V383 Lac are 6\% above the photosphere. These two stars have K0 spectral types with $J-K_{\mathrm{s}}$ colors of $0.53 \mathrm{mag}$, and therefore we adopted a low value for the intrinsic $R_{24 / 8}$. Since the photospheric value of $R_{24 / 8}$ appears to increase toward later spectral types (see Figure 4), the apparent photometric excess could be explained if we have underestimated the intrinsic value of $R_{24 / 8}$ by more than $0.6 \%$. This is possible given our simplistic treatment on how $R_{24 / 8}$ varies with the $J-K_{\mathrm{s}}$ color. The star W79 has a photometric $24 \mu \mathrm{m}$ excesses of $\sim 22 \%$ above the photosphere at a $\mathrm{S} / \mathrm{N}$ of $\sim 6.5$. We estimate that the apparent $24 \mu \mathrm{m}$ excess should have been detected in the IRS spectra between 24 and $34 \mu \mathrm{m}$ at $\geqslant 4.4 \sigma$ for dust temperature $\leqslant 100 \mathrm{~K}$. The $24 \mu \mathrm{m}$ image for W79 contains extended and structured cirrus emission that complicates background subtraction and could conceivably create an apparent excess. Given the discrepant results between the IRS spectra and MIPS $24 \mu \mathrm{m}$ for W79, V343 Nor, and V383 Lac, and that R45 is at the limit to identify a photometric excess but does not have a confirming spectrum, we do not consider these four sources to have MIPS $24 \mu \mathrm{m}$ excesses in the remainder of this paper.

We thus have 46 sources with both a $24 \mu \mathrm{m}$ and IRS excess. Spectra for 40 of these sources are presented in Figure 7; spectra for five sources appear in Bouwman et al. (2008) in a study of optically thick disks in the FEPS sample, and 1RXS J051111.1+281353 is presented in Figure 3. The detection of an infrared excess in both MIPS and IRS does not ensure the dust emission associated with the star, since contamination by interstellar cirrus and galaxies will affect both measurements. The expected extragalactic contamination can be assessed from the observed extragalactic counts as a function of $24 \mu \mathrm{m}$ flux density from Papovich et al. (2004). For each star, we computed the probability that at least one galaxy is present within the FWHM size $\left(6^{\prime \prime}\right)$ of the MIPS $24 \mu \mathrm{m}$ point response function that will produce $\mathrm{a} \geqslant 3 \sigma$ photometric excess at $24 \mu \mathrm{m}$. The photospheric $24 \mu \mathrm{m}$ fluxes were computed from the observed $8 \mu \mathrm{m}$ flux density and the adopted photospheric values of $R_{24 / 8}$ (see Section 3.4). We find that $\sim 1$ FEPS source could be contaminated by an extragalactic source bright enough to produce an apparent $24 \mu \mathrm{m}$ excess.

In Paper I, we considered the positional coincidence of the MIPS $24 \mu \mathrm{m}$ sources with the stellar coordinates to search for potential contaminants. For this sample of 314 stars, the largest astrometric offset between an MIPS $24 \mu \mathrm{m}$ excess source and the 2MASS stellar position is $1^{\prime \prime} 3$ for HD 201219, which is a $2.5 \sigma$ deviation based on the observed dispersion in the coordinate offsets. (Two sources without $24 \mu \mathrm{m}$ excesses had larger angular offsets.) The $70 \mu \mathrm{m}$ detection of HD 201219 is 

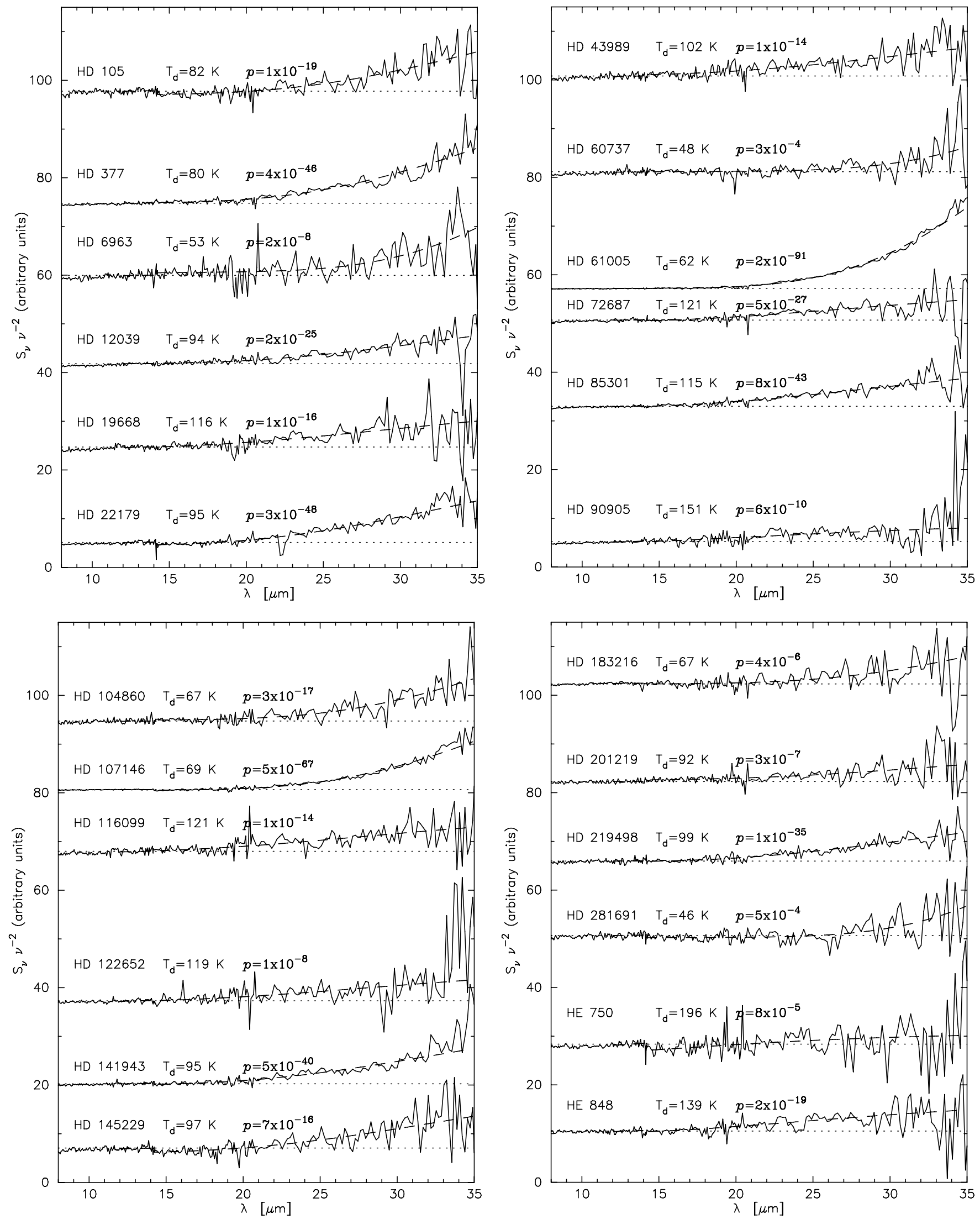

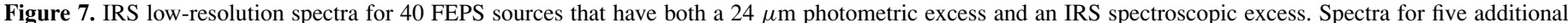

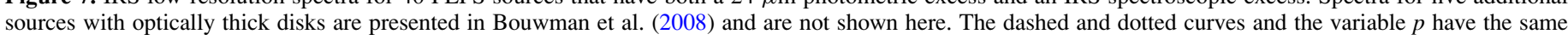
meaning described in Figure 2. The dust temperature $\left(T_{d}\right)$ inferred from the modified-blackbody fit $(\beta=0.8)$ is indicated for each source. 

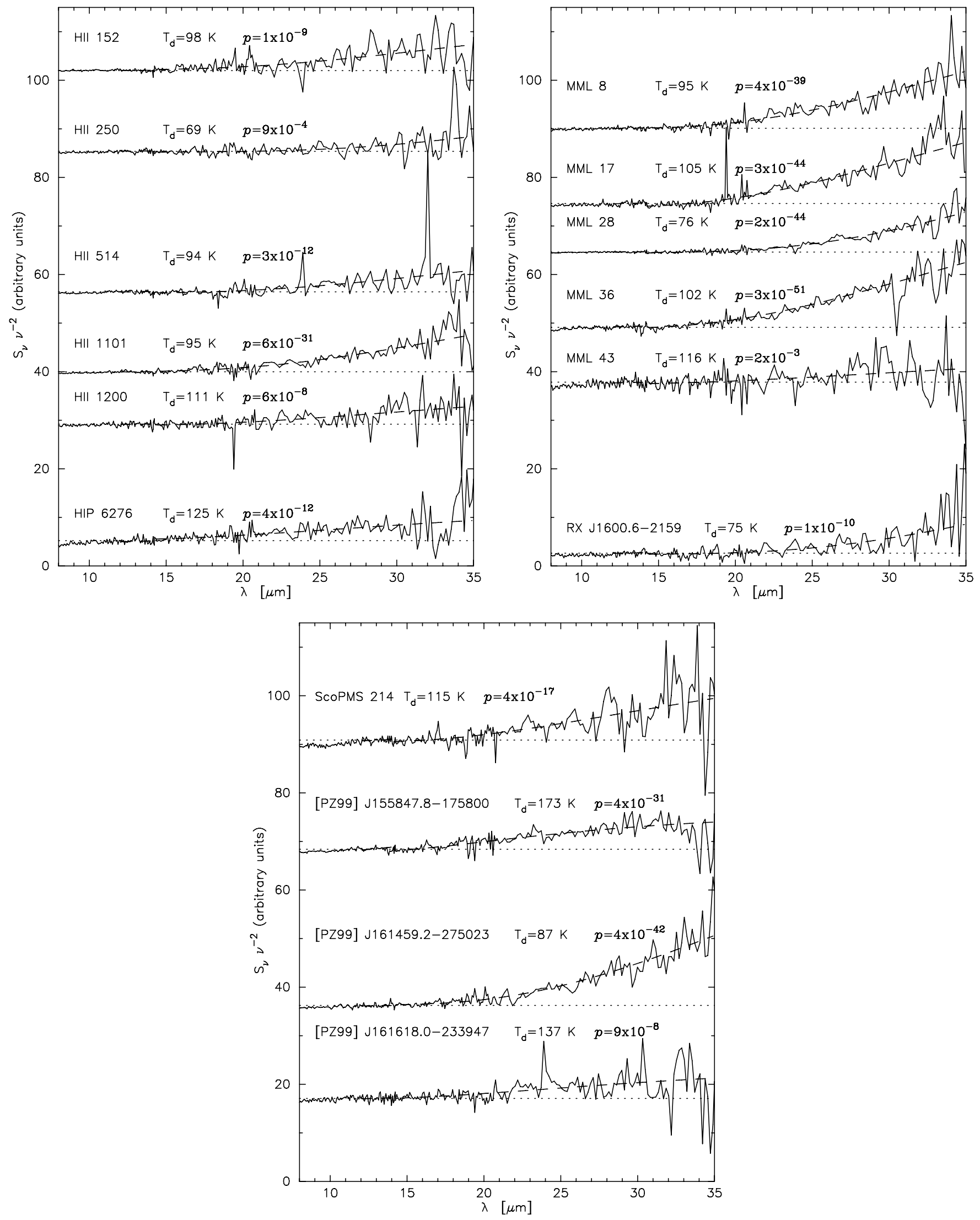

Figure 7. (Continued) 


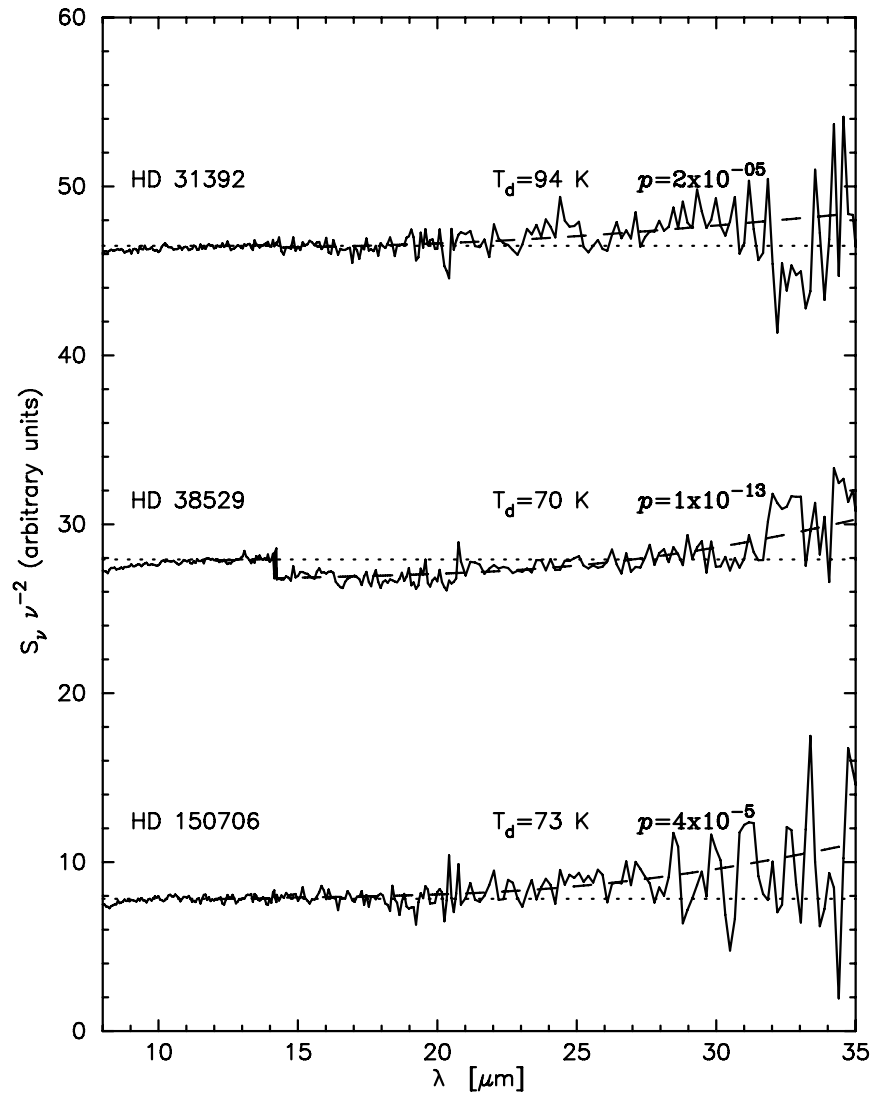

Figure 8. Same as Figure. 7, but for three sources with MIPS $70 \mu \mathrm{m}$ and IRS excesses and no MIPS $24 \mu \mathrm{m}$ excess.

offset by $3^{\prime \prime} 4$ from the stellar position. We consider this source to have an excess, but the photometry should be viewed with some caution. We conclude that while the photometry for $\sim 1$ FEPS source may be contaminated by a galaxy, there are no clear instances of contamination to the $24 \mu \mathrm{m}$ photometry ${ }^{14}$.

The reliability of sources with $70 \mu \mathrm{m}$ excesses can be assessed in a similar manner. Of the 21 sources with $\geqslant 3 \sigma$ excess at $70 \mu \mathrm{m}, 17$ also have a $24 \mu \mathrm{m}$ and IRS excess. Three of the remaining four $70 \mu \mathrm{m}$ excess sources have an IRS excess but not a $24 \mu \mathrm{m}$ excess; spectra for these sources are presented in Figure 8 . The one $70 \mu \mathrm{m}$ excess source without a confirming 24 $\mu \mathrm{m}$ or IRS excess is HD $187897(p=0.2)$, where the S/N of the $70 \mu \mathrm{m}$ excess is 6 . The detected $70 \mu \mathrm{m}$ sources are within $5^{\prime \prime}$ of the stellar position, and the expected number of contaminants from extragalactic sources that are nearly centered on the stellar source is negligible (Hillenbrand et al. 2008). We anticipate that most, if not all, of these sources are real $70 \mu \mathrm{m}$ excesses associated with the stellar target.

While the infrared excesses in most sources are confirmed with two or more Spitzer instruments, 23 sources have an excess detected with IRS that is not substantiated by either $24 \mu \mathrm{m}$ or $70 \mu \mathrm{m}$ photometry. The apparent IRS excesses for two sources (HD 104467 and RX J1531.3-3329) are deemed spurious since the inferred IRS dust temperatures are less than $14 \mathrm{~K}$, and the model $70 \mu \mathrm{m}$ flux density should have been readily detected

\footnotetext{
14 Stauffer et al. (2005) concluded that the measured $24 \mu \mathrm{m}$ excesses toward $\mathrm{H}$ II 152 and H II 250 were likely caused by background galaxies based on the positional mismatches between 2 MASS and the $24 \mu \mathrm{m}$ Spitzer image from pipeline version S10.5. However, the positional differences in the Spitzer data reduction pipeline version S13 were not significant (see Paper I) and we consider these apparent excesses to be real.
}

$(\gg 3 \sigma)$ if the excess was real. For the other 21 sources, the model $70 \mu \mathrm{m}$ density would have been detected at less than $2 \sigma$ and the MIPS observations cannot rule out the IRS excess. Spectra for these 21 sources are presented in Figure 9.

To investigate whether or not these sources likely contain real IRS excesses, in Figure 10 we compare the $\mathrm{S} / \mathrm{N}$ of the MIPS $24 \mu \mathrm{m}$ excess for sources with unconfirmed IRS excesses (black histogram) and without a detectable excess in any of the Spitzer instruments (gray histogram). Sources with unconfirmed IRS excesses tend to have larger S/Ns for the MIPS $24 \mu \mathrm{m}$ excess than sources without IRS excesses. Comparison of the dereddened $J-K_{\mathrm{s}}$ colors for the two samples using the Kolmogorov-Smirnoff $(\mathrm{K}-\mathrm{S})$ test indicates the two samples have indistinguishable distributions of $J-K_{\mathrm{s}}$ colors $(\mathrm{K}-\mathrm{S}$ probability $=0.24)$, indicating that the differences in the excess distributions is not a result of a systematic error in the assumed intrinsic colors. By contrast, the K-S probability that the two populations have the same signal-to-noise distribution of $24 \mu \mathrm{m}$ excesses is $10^{-6}$, which suggests that it is unlikely the positive bias to the $24 \mu \mathrm{m}$ excesses can be attributed to random noise. Moreover, if the IRS-only excesses were due to random noise, the age distribution of these sources should mimic that of the full sample. Instead, 19 of the 21 sources with IRS-only excesses are younger than $300 \mathrm{Myr}$, while 202 of the 314 stars in the full sample are this young. The probability that this difference in the age distributions could result by chance is $1.6 \%$. These results suggest that many of the IRS-only excess sources likely have a real infrared excess, but the $24 \mu \mathrm{m}$ photometric excess is too weak to detect.

In summary, we identified 50 stars that are considered to have a reliable infrared excess: 46 stars have both MIPS $24 \mu \mathrm{m}$ $(\geqslant 3 \sigma)$ and IRS excesses $(p \leqslant 0.003)$, three stars have both IRS and MIPS $70 \mu \mathrm{m}$ excesses $(\geqslant 3 \sigma)$ but no MIPS $24 \mu \mathrm{m}$ excess, and one star has a $70 \mu \mathrm{m}$ excess only. Of these 50 stars, 45 are considered "debris" disks and 5 are "primordial" disks (see Section 4). An additional 21 stars have an apparent IRS excess that is unconfirmed photometrically, but many of these sources likely have an excess based on the tendency to have positive $24 \mu \mathrm{m}$ photometric excesses (but less than $3 \sigma$ ).

\section{TEMPORAL EVOLUTION OF DEBRIS DISK PROPERTIES}

The results presented in Figures 1 and Figures 7 and 8 suggest a dichotomy in disk properties in that only five sources have excess emission at wavelengths $\leqslant 8 \mu \mathrm{m}$, while for the remaining sources the excesses appear only at longer ( $>16 \mu \mathrm{m}$ ) wavelengths. The five sources with $8 \mu \mathrm{m}$ excesses have properties (e.g., circumstellar disk masses, $\mathrm{H} \alpha$ accretion signatures, shape of the spectral energy distribution, high fractional disk luminosities) consistent with optically thick accretion disks (Silverstone et al. 2006; Bouwman et al. 2008). We show in Section 4.4 that the remaining sources have fractional infrared excess luminosities of $L_{\mathrm{IR}} / L_{*} \lesssim 10^{-3}$ and are consistent with optically thin dust emission. We assume that the thick disks represent "primordial" disks formed during the star-formation process, and the optically thin systems are "debris" disks, although the transition between these states is not well characterized observationally.

In this section, we establish the empirical signature of disk evolution within the FEPS sample from this sample of debris disks. Since no debris disks were detected at $8 \mu \mathrm{m}$, we investigate any evolutionary trends using MIPS photometry and 

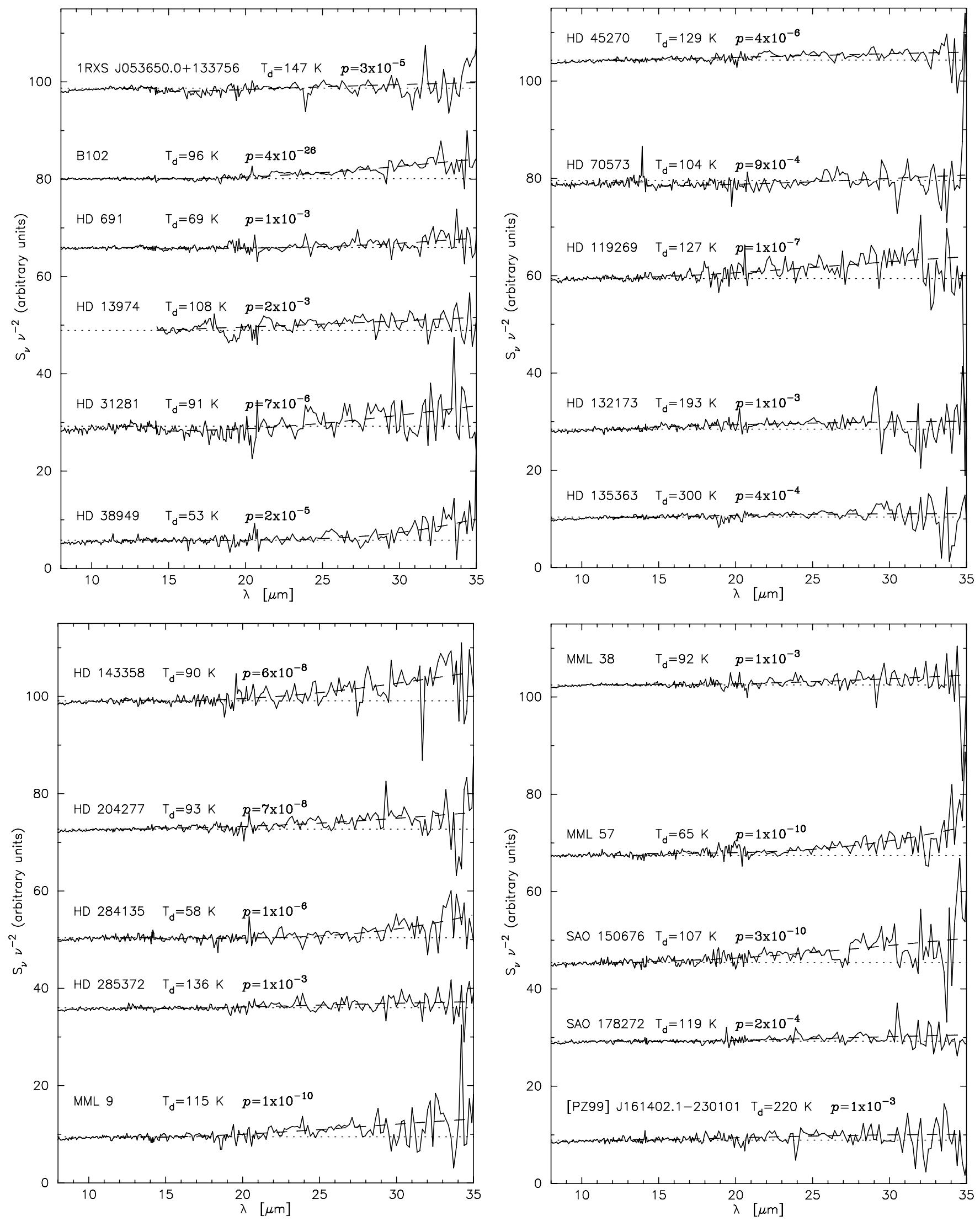

Figure 9. Same as Figure 7, but for 21 sources with IRS excesses and no detectable MIPS 24 or $70 \mu \mathrm{m}$ excess. 


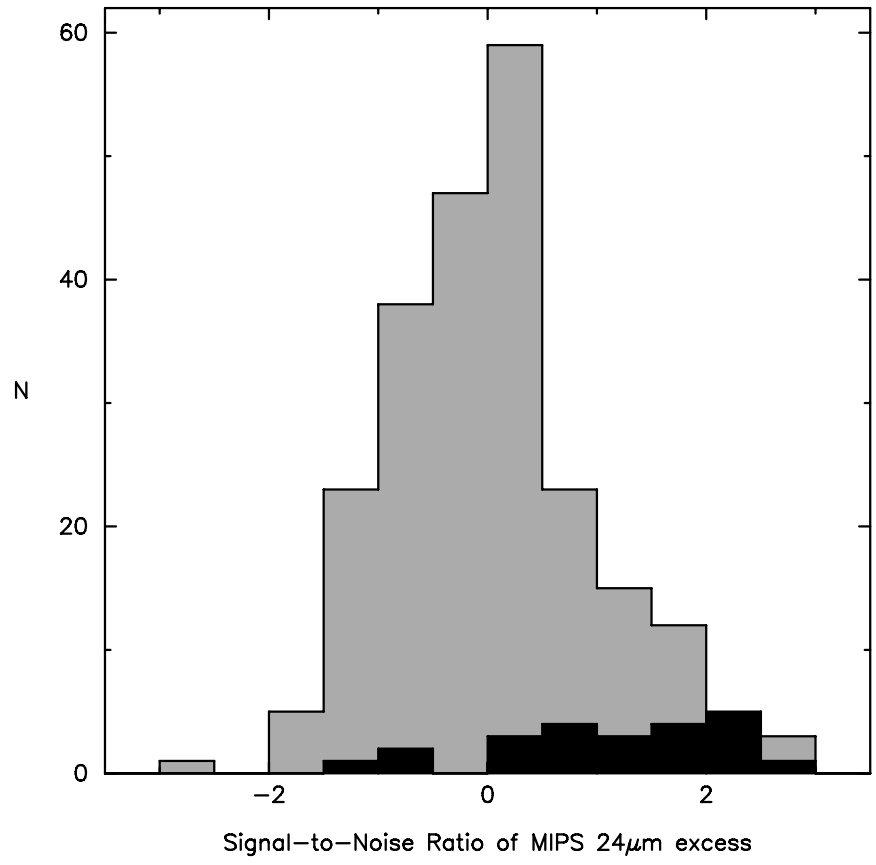

Figure 10. Histograms of the MIPS $24 \mu \mathrm{m}$ excess $\mathrm{S} / \mathrm{N}$ for subsets of the FEPS sample. The black histogram represents stars that have $\mathrm{a} \geqslant 3 \sigma$ excess from IRS spectra, but the excess is not confirmed with MIPS 24 or $70 \mu \mathrm{m}$ photometry. The gray histogram indicates sources that have less than a $3 \sigma$ excess in the IRS, MIPS $24 \mu \mathrm{m}$, and MIPS $70 \mu \mathrm{m}$ data. Sources with unconfirmed IRS excesses tend to have positive S/Ns for the $24 \mu \mathrm{m}$ excess, suggesting that the IRS excess is indeed real for many of these sources.

IRS spectra. We first examine if the FEPS sample of debris disks contains any bias with respect to stellar luminosity, since dust emission will be brighter around more luminous stars for a given dust surface area and orbital radius. Stars with detected debris disks have spectral types ranging from K3 to F5 and span an order of magnitude in stellar luminosity. The median stellar luminosity for stars with $24 \mu \mathrm{m}$ excesses is $1.15 L_{\odot}$, compared to the median luminosity of $1.07 L_{\odot}$ for the entire sample. The K-S test indicates a $77 \%$ probability that the stellar luminosities for stars with and without $24 \mu \mathrm{m}$ excesses are drawn from the same parent population. A similar probability was derived for stars with and without $70 \mu \mathrm{m}$ excesses. Thus there is no evidence for luminosity bias within the FEPS debris disk sample, and we use these stars to investigate trends in the debris properties.

\section{1. $24 \mu m$ Excesses}

In Figure 11, we show the ratio of observed-to-photospheric $R_{24 / 8}$ versus stellar age to investigate the temporal evolution of $24 \mu \mathrm{m}$ excess emission. The magnitude of the $24 \mu \mathrm{m}$ excesses ranges from a low of $5.9 \%$ above the photosphere, as limited by the accuracy of the photometric calibrations, to a high of $118 \%$ for HD $61005 .{ }^{15}$ The decline in the magnitude of the excess toward older ages appears abrupt in that the upper envelope of $24 \mu \mathrm{m}$ excesses is roughly flat at $\sim 50 \%$ above the photosphere (with HD 61005 as the main outlier) for ages $\lesssim 300 \mathrm{Myr}$, and $\sim 10 \%$ above the photosphere for ages $>$ 500 Myr. Based on the Kendall's rank correlation statistic, as implemented in the ASURV Rev 1.2 package (Lavalley et al. 1992), the probability that the apparent correlation between

15 Hines et al. (2007) present resolved scatter light images of the HD 61005 debris system obtained with the Hubble Space Telescope.

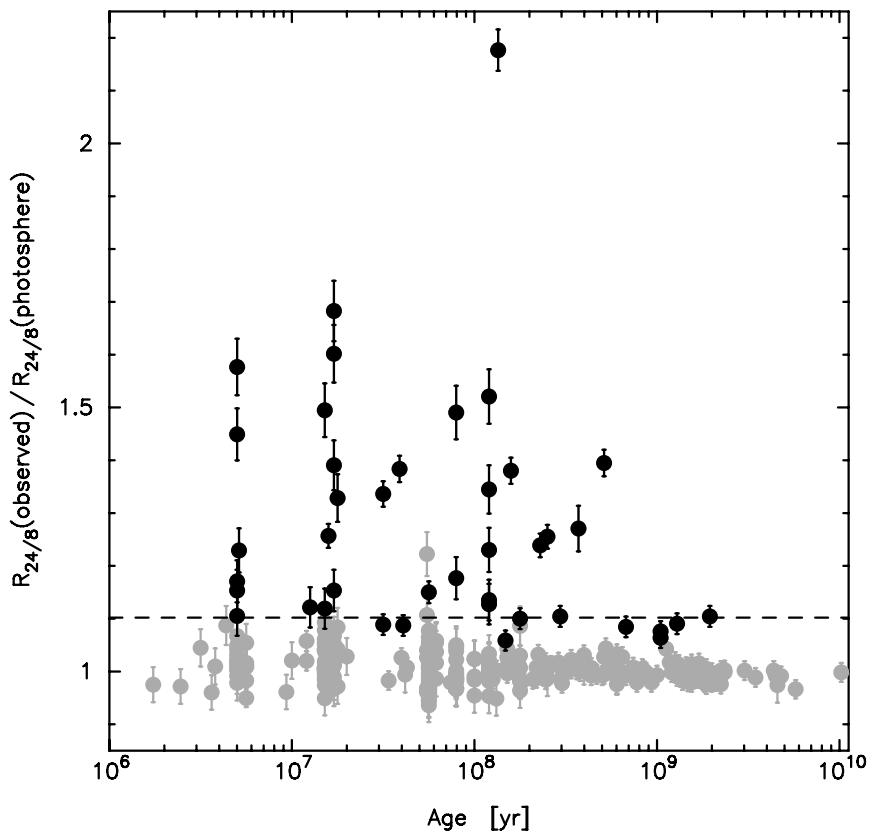

Figure 11. The ratio of the observed MIPS $24 \mu \mathrm{m}$ flux to $24 \mu \mathrm{m}$ photospheric flux as a function of stellar age. The filled symbols represent the 45 sources that have a $24 \mu \mathrm{m}$ photometric excess confirmed by an IRS spectrum. The dashed line at a value of 1.102 shows the minimum $24 \mu \mathrm{m}$ excess that could be detected at the $\geqslant 3 \sigma$ level over all stellar ages. Five sources with excesses from optically thick circumstellar disks are offscale on this plot.

stellar age and the magnitude of the $24 \mu \mathrm{m}$ excess can result from chance is $4 \times 10^{-5}$.

To further quantify the temporal evolution of the $24 \mu \mathrm{m}$ excess, we select a uniform sample of excess sources over all ages. While the IRS spectra are sensitive to smaller excesses, the $24 \mu \mathrm{m}$ photometry is more uniform and is available for the full FEPS sample. Therefore, we draw a uniform sample based upon the $24 \mu \mathrm{m}$ photometry. The precision of $R_{24 / 8}$ is poorest for sources with $S_{v}<100 \mathrm{mJy}$, where $\sigma\left(R_{24 / 8}\right) / R_{24 / 8}=$ $3.4 \%$ (see Section 3.4). The minimum $24 \mu \mathrm{m}$ excess that can be detected over all stellar ages at $\geqslant 3 \sigma$ is $10.2 \%$. A total of 38 sources have a $24 \mu \mathrm{m}$ photometric excess greater than this limit, of which five are primordial disks and 33 are debris disks.

In Figure 12, we show the fraction of debris disks that have a $24 \mu \mathrm{m}$ excess greater than $10.2 \%$ versus stellar age. The age bins, selected to span a factor of 3 in age, are younger than $10 \mathrm{Myr}$, between 10 and $30 \mathrm{Myr}, 30-100 \mathrm{Myr}, 100-300 \mathrm{Myr}$, 300-1000 Myr, and older than 1 Gyr. Preliminary results of this analysis were presented in Meyer et al. (2008), but we use the combined MIPS and IRS data to select a more reliable sample of sources with $24 \mu \mathrm{m}$ excesses. The results presented in Figure 12 and tabulated in Table 4 are consistent with the $24 \mu \mathrm{m}$ excess fraction remaining constant to within the uncertainties at a mean of $15 \%$ for ages less than 300 Myr. For older ages, the excess fraction declines to a mean of $2.7 \%$. Using the twotailed Fisher's exact test to compare ratios, the probability that the $24 \mu \mathrm{m}$ excess fraction is the same for ages younger and older than $300 \mathrm{Myr}$ is $0.04 \%$. This result indicates a decline in the $24 \mu \mathrm{m}$ excess fraction with stellar age. However, the stellar age uncertainties prevent us from determining the form of the decline. As noted in Meyer et al. (2008), age uncertainties will tend to soften the decline perhaps indicating that it is starker than observed here. 


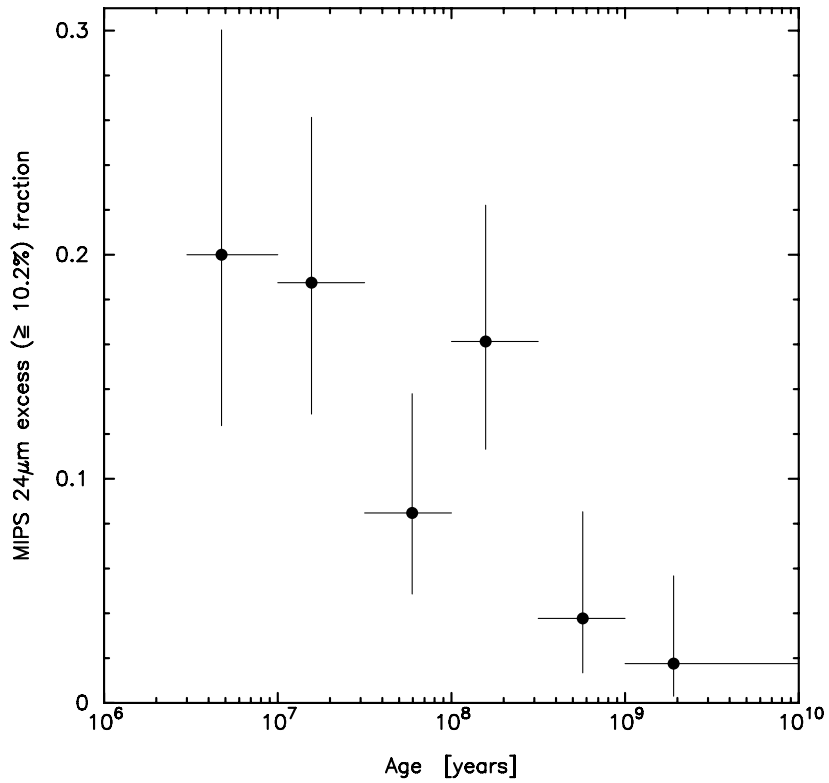

Figure 12. Fraction of sources with an MIPS $24 \mu \mathrm{m}$ excess greater than $10.2 \%$ of the photosphere, and verified with the IRS spectrum, as a function of age. Five sources with optically thick disks at ages $<12 \mathrm{Myr}$ are not included to show only the temporal evolution from optically thin debris dust. Vertical error bars represent the $1 \sigma$ uncertainties computed from binomial statistics using the tables in Gehrels (1986). Horizontal "error" bars are the range of stellar ages in the corresponding bin, where the points are placed at the mean age in the bin.

Table 4

$24 \mu \mathrm{m}$ Excess Fraction Versus Age ${ }^{\mathrm{a}}$

\begin{tabular}{lcccc}
\hline \hline Age Range & Mean Age & \multicolumn{2}{c}{ Number of Stars } & Excess Fraction \\
\cline { 3 - 4 }$(\mathrm{Myr})$ & $(\mathrm{Myr})$ & Excess & Total & \\
\hline$<10$ & 5 & 6 & 30 & $0.20_{-0.08}^{+0.10}$ \\
$10-30$ & 16 & 9 & 48 & $0.19_{-0.06}^{+0.07}$ \\
$30-100$ & 59 & 5 & 59 & $0.085_{-0.04}^{+0.05}$ \\
$100-300$ & 150 & 10 & 60 & $0.17_{-0.05}^{+0.06}$ \\
$300-1000$ & 560 & 2 & 55 & $0.036_{-0.024}^{+0.046}$ \\
$\geqslant 1000$ & 1800 & 1 & 57 & $0.018_{-0.015}^{+0.039}$ \\
\hline
\end{tabular}

Notes.

a Values computed for a $10.2 \%$ excess above the photosphere and exclude five sources with optically thick disks.

Because the Fisher's exact test considers only the binomial distribution of the excess fraction and does not account age uncertainties, we conducted a Monte Carlo experiment where the estimated stellar ages were modified randomly within a Gaussian distribution, and the fraction of stars with $24 \mu \mathrm{m}$ excesses younger and older than 300 Myr was re-evaluated. For stars that are members of associations or clusters, we assumed an age uncertainty of $\sigma[\log ($ age $)]=0.15$. For field stars and members of the Corona Australis association, we assumed uncertainty $\sigma[\log ($ age $)]=0.3$. A larger uncertainty for Corona Australis was adopted since it represents our youngest association where not only is the fractional error in age likely larger than for the older associations and clusters, but a true range of stellar ages may indeed be present as perhaps indicated by the mixture of stars without any disks and with optically thick disks. Our adopted age uncertainties are ad hoc, but we believe that they represent reasonable estimates. In 10,000 simulations, the number of stars younger than $300 \mathrm{Myr}$ with $24 \mu \mathrm{m}$ excesses exceeded the number of such excess sources older than
$300 \mathrm{Myr}$ by at least a factor of 2 in $99 \%$ of the trials. Even if the age uncertainty for all stars was as $\operatorname{large}$ as $\sigma[\log (\operatorname{age})]=0.5$, the excess fraction is larger than older stars in $99.6 \%$ of the trials. We conclude that the temporal decline in the fraction of stars with $24 \mu \mathrm{m}$ excesses is robust to plausible, random age uncertainties.

The sample of stars younger than $300 \mathrm{Myr}$ contain a mixture of stars in clusters $(20 \%)$, associations $(28 \%)$, and the field (52\%), while $80 \%$ of the sample older than $300 \mathrm{Myr}$ are field stars. Clusters are typically considered to have stable dynamical times longer than $100 \mathrm{Myr}$ while associations disperse into the field on timescales less than this (e.g., Lada \& Lada 2003). Environment, either in the form of high initial stellar density in clusters or the high radiation field from any OB stars in clusters or associations, could impact the lifetime of primordial disks and ultimately the formation of debris systems. We examine then whether or not the declining $24 \mu \mathrm{m}$ excess fraction with age is a result of differences in the excess properties in cluster, association, and field-star populations. ${ }^{16}$

For stars younger than $300 \mathrm{Myr}$, the fraction of stars that have a $24 \mu \mathrm{m}$ excess $\geqslant 10.2 \%$ above the photosphere is $7 / 38$ $(18 \%), 11 / 56(20 \%)$, and 12/103 (12\%) for clusters, associations, and field stars, respectively. Given the similar percentages for clusters and associations, we combine those samples into one. While the excess fraction for clusters/associations is nearly twice as high as for field stars, the probability that the field star excess fraction is drawn from the same parent population as cluster/associations is $17 \%$ based on the Fisher's exact test. Therefore we are unable to determine definitively if a significant difference exists in the excess fraction between the two populations. We also considered the evolution of cluster/association stars and field stars separately. The probability that the excess fraction of cluster/association members younger than $300 \mathrm{Myr}$ is the same as for older cluster stars $(0 / 22)$ is $2 \%$. Similarly, there is a $6 \%$ chance that the excesses fraction in young and old field stars was drawn from the same excess parent population. The fact that the decline in the excess fraction is suggestive for both clusters/associations and field stars, albeit at weak confidence for the individual samples, suggests that the overall decline in the excess fraction with age results from temporal evolution and not a change in the mix of clusters/association and field star populations.

Our results can be compared with other Spitzer surveys of debris disks. Since each survey adopts different detection thresholds and sample selection, this comparison is qualitative in nature. Siegler et al. (2007) compiled various Spitzer 24 $\mu \mathrm{m}$ surveys of FGK stars and found a decline in the $24 \mu \mathrm{m}$ excess fraction with age similar to that observed in the FEPS sample. However, they inferred a higher excess fraction $(36 \%$ on average) in clusters younger than $50 \mathrm{Myr}$ compared to the FEPS sample despite having a higher detection threshold for a $24 \mu \mathrm{m}$ excess $(15 \%)$. The frequency of debris disks found around A stars declines on similar timescales as solar-type stars, although the debris disk frequency is higher (Rieke et al. 2005). One should keep in mind that debris disks identified toward A-type stars as a relative fraction of the stellar photospheric emission possess greater dust masses than similarly selected debris disks around $\mathrm{G}$ stars. Further, the dust detected at a given temperature generating $24 \mu \mathrm{m}$ excess is located at a greater orbital radius around A stars compared to G stars. Currie et al. (2008) suggest

\footnotetext{
16 Many of the field stars could have formed in a cluster or association that has since dispersed. However, we have no means to distinguish dispersed cluster or association stars from sources that actually formed in relative isolation.
} 


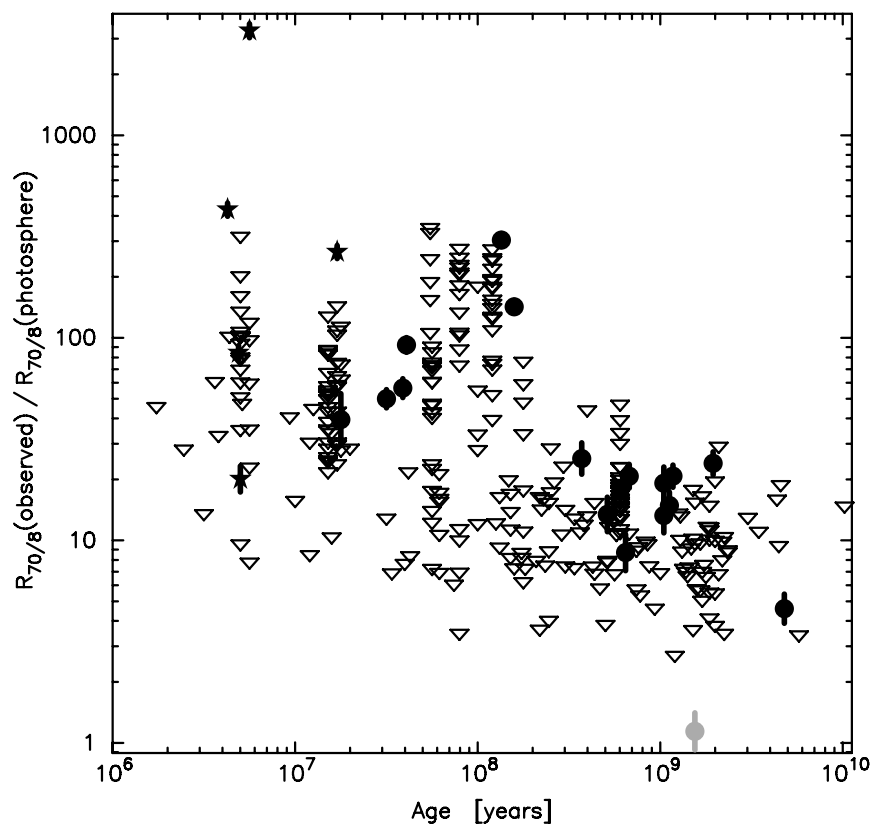

Figure 13. The ratio of the observed MIPS $70 \mu \mathrm{m}$ flux density to the $70 \mu \mathrm{m}$ photospheric value as a function of the stellar age. The filled symbols represent sources that have $\mathrm{a} \geqslant 3 \sigma 70 \mu \mathrm{m}$ detection, with stars representing optically thick disks, the black circles indicating optically thin debris disks, and the gray circle marking the source with a $70 \mu \mathrm{m}$ detection consistent with photospheric emission. The open triangles represent the $3 \sigma$ upper limits for sources not detected at $70 \mu \mathrm{m}$.

a more complicated evolutionary picture where the magnitude of the $24 \mu \mathrm{m}$ excess for A- and F-type stars increases from 5 to $10 \mathrm{Myr}$, peaks at ages of $10-15 \mathrm{Myr}$, and then declines toward older ages. Based on the Kendall's rank correlation statistic and visual inspection of Figure 11, we do not find a similar trend for solar-type stars.

\section{2. $70 \mu \mathrm{m}$ Excesses}

We present in Figure 13 the ratio of the observed-tophotospheric $70 \mu \mathrm{m}$ flux density as a function of stellar age. For sources that were not detected at $70 \mu \mathrm{m}$, upper limits to the observed flux density were computed as $3 \sigma$ if $S_{v}(70 \mu \mathrm{m}) \leqslant 0$, or as $S_{v}(70 \mu \mathrm{m})+3 \sigma$ if $S_{v}(70 \mu \mathrm{m})>0$. The upper limits include both internal and calibration uncertainties. The upper limits to the $70 \mu \mathrm{m}$ emission are typically 3-30 times the photosphere for stars older than $\sim 300 \mathrm{Myr}$, and 10-200 times for the younger, more distant stars. The detected $70 \mu \mathrm{m}$ sources have excesses up to $300 \times$ and $3000 \times$ the photosphere for the debris and primordial disks, respectively.

The maximum fractional $70 \mu \mathrm{m}$ excess occurs between ages of $\sim 30$ and $200 \mathrm{Myr}$. Of the 105 stars in this age range, at least five sources have a $70 \mu \mathrm{m}$ fractional excess more than 50 times the photosphere. By contrast, of the 126 stars older than $200 \mathrm{Myr}$, none have this large of an excess and the upper limits to the $70 \mu \mathrm{m}$ fractional excess are all below 50. The probability that the luminous excess sources in the two ages samples are drawn from the same parent population is $1.8 \%$ according to the Fisher's exact test. However, the significance of this comparison is sensitive to the age range chosen. If we compare all stars younger than $300 \mathrm{Myr}$ to the older stars, the probability that the luminous excess are down from the same parent population is $8 \%$. We also used the Kendall's rank correlation statistic to evaluate if the $70 \mu \mathrm{m}$ excess depends on stellar age. Considering both detections and upper limits to the $70 \mu \mathrm{m}$ excess, the probability of a trend of $70 \mu \mathrm{m}$ excess emission with age is $46 \%$.

The results presented in Figure 13 possibly suggests that relatively few luminous $70 \mu \mathrm{m}$ excess sources indicative of debris dust are found among the youngest sources. Considering only sources younger than $200 \mathrm{Myr}$, the Kendall's rank correlation statistic indicates a $84 \%$ probability that a correlation is present the $70 \mu \mathrm{m}$ excess with age. We therefore do not find any evidence for evolution in the debris luminosity between 3 and 200 Myr.

In summary, we find weak evidence for a decline in the magnitude of the peak $70 \mu \mathrm{m}$ excess from intermediate-age stars (30-200 Myr) to older ages. We do not find any systematic temporal change in the overall $70 \mu \mathrm{m}$ excess, or if the amount of $70 \mu \mathrm{m}$ emission evolves between stellar ages of 3 and 200 Myr. Observations of additional stars in this age range combined with more sensitive flux density limits are needed to make more definitive conclusions. Compared to debris disks around A star, Su et al. (2006) found that the decay time of the $70 \mu \mathrm{m}$ emission is $\sim 400$ Myr. Qualitatively that is consistent with the FEPS data in that the upper envelope of $70 \mu \mathrm{m}$ emission appears to decline between 100 and 300 Myr.

\subsection{Dust Temperature}

Assuming the dust grains are in thermal equilibrium with the stellar radiation field, the dust temperature is a first order indicator of the orbital radius. For grains that are efficient absorbers of stellar radiation, the orbital radius, $R$, is proportional to $R \propto L_{*}^{0.5} T_{\text {dust }}^{-\frac{4+\beta}{2}}$, where $L_{*}$ is the stellar luminosity and $T_{\text {dust }}$ the dust temperature. In practice, the orbital radius cannot be derived uniquely from spectral energy distributions without knowledge of the grain size, composition, and porosity. Nonetheless, variations in the dust temperature may translate into a range of orbital radii if the grain properties are similar amongst the debris disks.

Dust temperatures derived by fitting a Kurucz model plus a modified blackbody $(\beta=0.8)$ to the IRS spectra between 12 and $35 \mu \mathrm{m}$ (see Section 3.2) are plotted versus stellar age in the bottom panel in Figure 14 for debris disks with an IRS excess and either an MIPS 24 or $70 \mu \mathrm{m}$ excess. Different colored symbols are shown for sources with (black circles) and without (gray circles) $70 \mu \mathrm{m}$ excesses. A greater fraction of the older sources tend to have $70 \mu \mathrm{m}$ excesses, which most likely reflects that older stars tend to be closer in distance than the younger objects, and smaller excesses can be detected. The dust temperatures range from $46 \pm 7 \mathrm{~K}$ (HD 281691) to $196 \pm 48 \mathrm{~K}$ (HE 750) with a median of $112 \mathrm{~K}$. The distribution of dust temperatures overlaps for sources with and without $70 \mu \mathrm{m}$ detections, although the median dust temperature is higher for sources without $70 \mu \mathrm{m}$ detections (102 K vs. $81 \mathrm{~K}$ ). The top panel in Figure 14 shows the dust temperature derived from the MIPS 24 and $70 \mu \mathrm{m}$ photometry. The temperatures derived from the MIPS photometry are lower than that inferred from the IRS spectra, suggesting the presence of cooler dust (see Section 4.4). No strong trend between dust temperature and stellar age is evident.

The observed scatter in the ratio $L_{*}^{0.5} T_{\text {dust }}^{-2}$, which is proportional to the orbital radius assuming isothermal dust radiating as a blackbody, is three times larger than the expected scatter if the orbital radius was the same for all sources. These results suggest variations are present in the orbital location of dust. Variations in dust properties may also contribute to the scatter, but we 

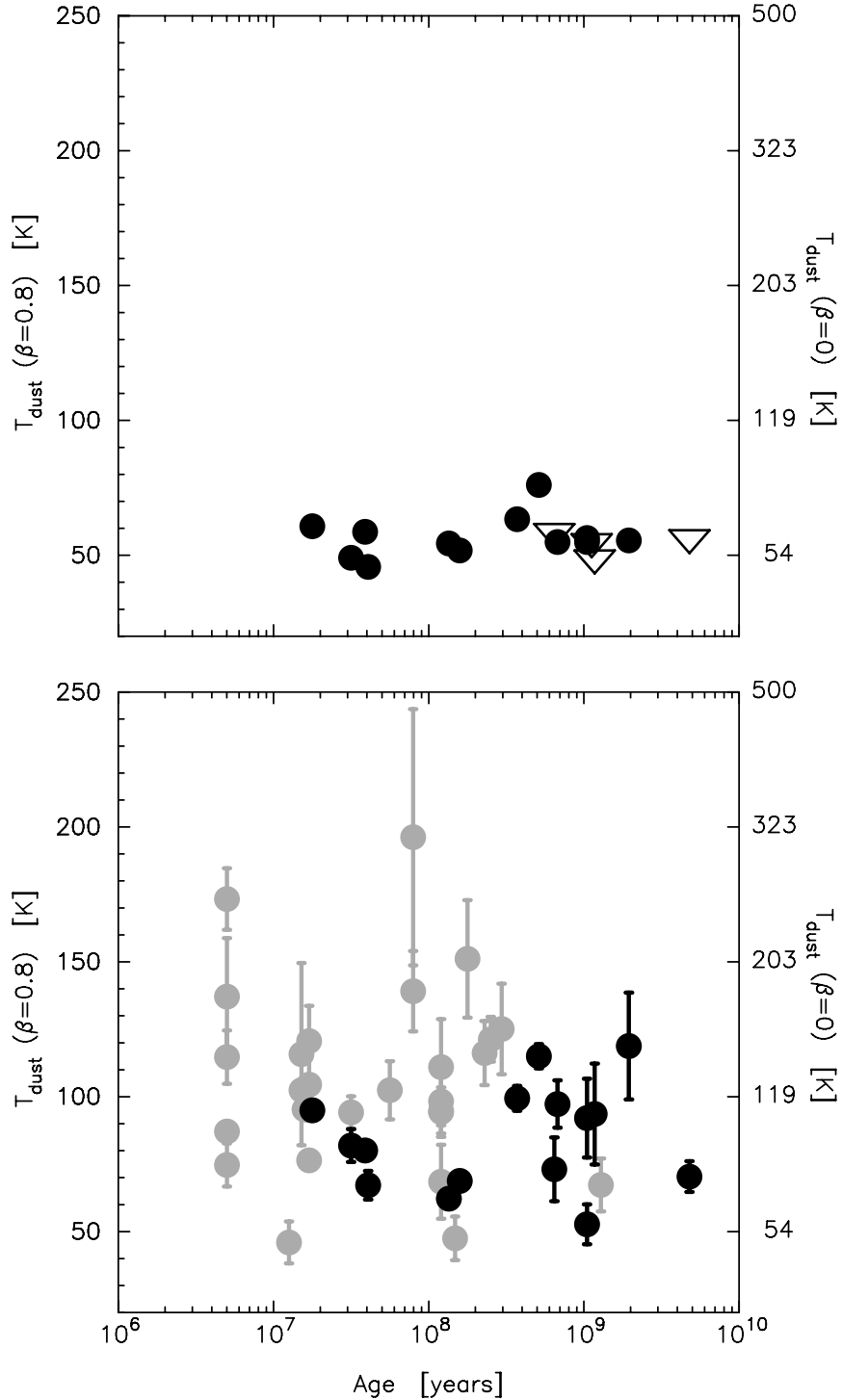

Figure 14. Dust temperatures as a function of stellar age for debris disks in the FEPS sample. The top panel shows temperatures derived from the MIPS 24 and $70 \mu \mathrm{m}$ photometry for 16 stars with $70 \mu \mathrm{m}$ excesses. The bottom panel shows temperatures inferred from IRS spectra for 43 debris disks with an IRS excess and either an MIPS 24 or $70 \mu \mathrm{m}$ excess. The IRS dust temperatures were derived assuming the excess can be approximated by a modified blackbody with $\beta=0.8$. Equivalent blackbody temperatures are shown on the right axis. Black and gray circles indicate sources with and without a detected $70 \mu \mathrm{m}$ excess, respectively.

consider here only the range of orbital radii implied by the observations. The minimum orbital radius of the dust grains can be estimated assuming the grains radiate like blackbodies. The implied orbital radius is $R=\left(L_{*} / L_{\odot}\right)\left(278 \mathrm{~K} / T_{\text {dust }}\right)^{2} \mathrm{AU}$. The range of blackbody temperatures derived from the IRS spectra, $50 \pm 9 \mathrm{~K}$ to $283 \pm 127 \mathrm{~K}$ with a median of $113 \mathrm{~K}$, corresponds to orbital radii of $31 \mathrm{AU}$ and $1 \mathrm{AU}$ with a median of $6 \mathrm{AU}$. (We excluded 1RXS J051111.1+281353 since the IRS excess is poorly fitted with a modified blackbody.) If most of the surface area is from smaller grains which do not emit as blackbodies, the corresponding radii will be larger.

\subsection{Fractional Dust Luminosity}

The amount of dust emission radiated in the $24 \mu \mathrm{m}$ and $70 \mu \mathrm{m}$ bandpasses depends on both the surface area and temperature of the dust grains. The fractional dust luminosity $\left(f_{\text {dust }}=L_{\text {dust }} / L_{*}\right)$, or equivalently the fractional dust bolometric flux $\left(f_{\text {dust }}=F_{\text {dust }} / F_{*}\right.$ ), accounts for variations in dust crosssectional surface area and temperatures to reflect the total amount of stellar emission absorbed and reradiated by dust grains.

The luminosity of "warm" dust emission between 12 and $35 \mu \mathrm{m}$ is constrained by the IRS spectra. The modifiedblackbody fits provide estimates of both the dust temperature and surface area of dust grains, and extrapolation of these fits yields the bolometric flux of warm dust $\left(F_{\text {warm }}\right)$ over all wavelengths. For sources without a detectable IRS excess, upper limits to $F_{\text {warm }}$ were computed by first integrating the excess emission in the IRS spectrum after subtracting the model photosphere and computing the formal uncertainty in the integrated flux. The $3 \sigma$ upper limits were then computed in a manner similar to that for the $70 \mu \mathrm{m}$ upper limits as described in Section 4.2. The warm dust bolometric flux, $F_{\text {warm }}$ was normalized by the photospheric bolometric flux $\left(F_{*}\right)$ estimated as

$$
F_{*}=\frac{\sigma_{\mathrm{SB}} T_{\mathrm{eff}}^{4} S_{v}(3.6 \mu \mathrm{m})}{\pi B_{v}\left(3.6 \mu \mathrm{m}, T_{\mathrm{eff}}\right)},
$$

where $\sigma_{\mathrm{SB}}$ is the Stefan-Boltzmann constant, $T_{\text {eff }}$ is the photospheric temperature estimated from the observed stellar colors or spectral type (see Paper I), $S_{v}(3.6 \mu \mathrm{m})$ is the observed IRAC $3.6 \mu \mathrm{m}$ flux density, and $B_{v}\left(\lambda, T_{\text {eff }}\right)$ is the Planck function.

The "cool" dust luminosity radiated at wavelengths longer than $35 \mu \mathrm{m}$ is not as well determined since we have a single observation at $70 \mu \mathrm{m}$ and most of our measurements are upper limits. Moreover, Hillenbrand et al. (2008) found that the color temperature inferred from the observed 33 to 24 $\mu \mathrm{m}$ flux densities is often higher than that derived from the 70 to $33 \mu \mathrm{m}$ flux density ratio, although the significance of the temperature difference is marginal for any single star. The modified blackbody fits to the IRS spectra provide a more accurate assessment of the dust temperature since the shape of the entire emission spectrum is used to estimate the dust temperature and luminosity. We find that the $70 \mu \mathrm{m}$ flux density from the modified blackbody fits underestimates the observed $70 \mu \mathrm{m}$ excess flux density by $3-11 \sigma$ for 11 of the 16 debris disks with a $70 \mu \mathrm{m}$ detection even if $\beta=0$ is assumed to maximize the predicted $70 \mu \mathrm{m}$ emission. For the five remaining debris disks, the observed flux density exceeds the projected value, but by less than $3 \sigma$. These results suggests that an additional cool dust component is present in many sources which contributes significantly to the $70 \mu \mathrm{m}$ emission but not at IRS wavelengths. The dust luminosities estimated from the IRS spectra then underestimate the total luminosity.

Given that the distribution of dust temperatures is poorly constrained by the $70 \mu \mathrm{m}$ observations, we adopt a twocomponent model to estimate the bolometric dust luminosity. The predicted $70 \mu \mathrm{m}$ flux density from the warm component was subtracted from the observed $70 \mu \mathrm{m}$ flux density to yield the $70 \mu \mathrm{m}$ emission from cooler dust $\left(S_{v}^{\mathrm{cool}}\right)$. The bolometric flux of cool dust was estimated assuming a single dust temperature, $T_{\text {cool }}$, as

$$
F_{\text {cool }}=\frac{S_{v}^{\mathrm{cool}}\left(\nu_{70}\right) \int v^{\beta} B_{v}\left(T_{\mathrm{cool}}, v\right) d v}{\nu_{70}^{\beta} B_{v}\left(T_{\mathrm{cool}}, \nu_{70}\right)},
$$

where $\nu_{70}$ is the frequency corresponding to the mean wavelength of the MIPS $70 \mu \mathrm{m}$ bandpass. We adopt $T_{\text {cool }}=60 \mathrm{~K}$ 
to conform to the temperature frequently inferred from midinfrared observations of debris disks around solar-type stars (Zuckerman \& Song 2004; Hillenbrand et al. 2008). Upper limits to the cool luminosity were computed using $T_{\text {cool }}$ and the upper limit to $F_{\text {cool }}$. The upper limits do not include the uncertainty in $T_{\text {cool }}$ nor the arbitrary amount of luminosity that could be generated by adding even cooler dust that does not radiate prominently at $70 \mu \mathrm{m}$.

The fractional dust luminosity as a function of stellar age is presented in Figure 15 for the warm dust, and the sum of the warm and cool dust components. The trends apparent in this figure are similar to those concluded based on the MIPS 24 and $70 \mu \mathrm{m}$ photometry considered alone. The fractional warm luminosity from the young (optically thick) disks is typically an order of magnitude greater than that from older (debris) disks, although the fractional luminosity from the strongest debris disk (HD 61005) is lower by only a factor of $\sim 5$. For sources with $70 \mu \mathrm{m}$ detections, the debris disk sources with the highest fractional dust luminosity are at ages younger than $200 \mathrm{Myr}$ with a decline in the peak fractional luminosity toward old ages. The fractional luminosities of the detected debris disks in the FEPS sample are similar to the solar-type stars of comparable age observed by Trilling et al. (2008). A-type stars show a similar decline in the fractional luminosity on timescales of 100-300 Myr (Su et al. 2006) as observed in the FEPS solarstar sample.

\section{DEBRIS DISK MODELS}

The dynamics of dust grains in optically thin systems are dominated by radiative and collisional processes when the effects of gas drag are negligible (Takeuchi \& Artymowicz 2001). Pascucci et al. (2006) used Spitzer high-resolution spectroscopy to place an upper limit of $0.04 M_{\text {Jupiter }}$ to the gas mass in the inner disk for 15 debris systems within the FEPS program having ages between 5 and 400 Myr. While these limits are still too high to establish whether gas drag is a marginal process in the evolution of dust grains (gas-to-dust ratio < 0.1 ), we assume here that is in fact the case.

For debris disks detectable with current instrumentation, Dominik \& Decin (2003) demonstrated that collisional processes combined with radiation pressure dominate over PoyntingRobertson drag in removing dust grains from the system (see also Wyatt 2005). Hillenbrand et al. (2008) confirmed that collisions are likely the dominant process for the disks detected at $70 \mu \mathrm{m}$ in the FEPS sample. Stellar mass loss also produces a drag on the orbiting dust in a manner analogous to the PoyntingRobertson effect, and for the Sun, the current rate of dust mass loss from corpuscular drag is about 0.3 that of radiation drag (Gustafson 1994). At younger ages the stellar mass-loss rates are likely higher, and wind drag may be more important than Poynting-Robertson drag and even collisions for young debris disks (Jura 2004; Chen et al. 2005). However, given the uncertainties on the scaling of mass-loss rates with stellar age (Wood et al. 2002, 2005), we do not consider wind drag effects.

Assuming that collisional processes are the dominant forces influencing dust dynamics, the location of the dust grains should trace the spatial distribution of the planetesimals. Therefore, we adopt a model in which the planetesimals are cospatial with the debris dust. The following section describes the model calculations, and in subsequent sections we apply this model to infer the properties and evolution of the planetesimals.

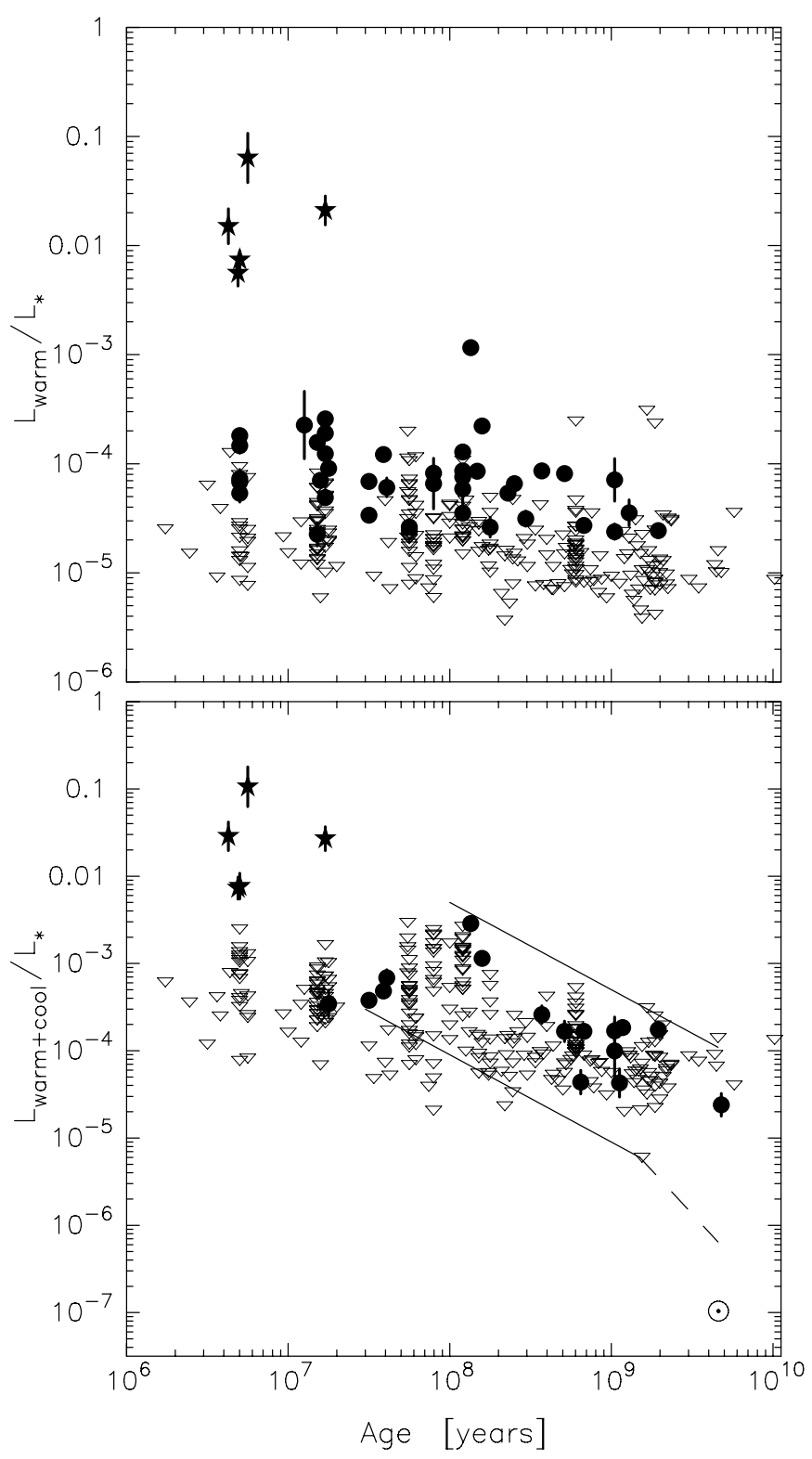

Figure 15. The ratio of the infrared excess to stellar luminosity for "warm" dust emission (upper panel) and the "warm" plus "cool" dust emission (bottom panel). The warm dust luminosity was computed from modified blackbody fits to the observed IRS spectra, with upper limits computed assuming a dust temperature of $100 \mathrm{~K}$. The warm plus cool dust luminosity was estimated from the IRS spectra and the $70 \mu \mathrm{m}$ flux assuming a cool dust temperature of $60 \mathrm{~K}$ (see text). The filled symbols represent sources that have a $\geqslant 3 \sigma 70$ $\mu \mathrm{m}$ photometric detection above the stellar photosphere, with stars and circles representing optically thick and thin disks, respectively. The open triangles represent the $3 \sigma$ upper limits for sources not detected at $70 \mu \mathrm{m}$. The solid lines in the bottom panel show $t^{-1}$ evolutionary curves appropriate for debris systems dominated by collisions; the curves are normalized to the brightest and faintest detected debris disks and are not a fit to the data. The dashed curve shows the expected evolution when the system becomes dominated by Poynting-Robertson drag (see text). The solar symbol indicates the luminosity of the Kuiper Belt (Backman et al. 1995).

\subsection{Model Description}

The model planetesimal belt extends between an inner orbital radius $R_{\text {in }}$ and an outer radius $R_{\text {out }}$, and contains particles with radii between $a_{\min }$ and $a_{\max }$. The particle size distribution as a function of orbital radius $(R)$ and particle radius $(a)$ is

$$
N(a, R)=K a^{-3.5} R^{\alpha}
$$


such that $N(a, R) d a d R$ is the number of particles between $a$ and $a+d a$, and between $R$ and $R+d R$. Since the slope of the particle size distribution is the same over all orbital radii in this model, the particle surface area and mass surface density will also scale as $R^{\alpha}$. The particle size distribution of $a^{-3.5}$ is appropriate for a collisional cascade without boundary conditions on the minimum and maximum particle sizes (Dohnanyi 1969). In practice, radiation pressure imposes limits on the minimum particle size, and numerical simulations predict a "wavy" pattern of particle sizes since the smallest grains are not available to break up particles near the cutoff limit (Campo Bagatin et al. 1994; Thébault \& Augereau 2007). For simplicity, however, we consider only a power-law distribution of particle sizes. With this adopted size distribution, most of the grain surface area is contained in the smallest particles such that $a_{\min }$ is the critical parameter, while the larger particles contain most of the disk mass $\left(M_{\text {disk }} \propto \sqrt{a_{\text {max }}}\right)$.

The flux density from the disk assuming optically thin emission is

$$
\begin{aligned}
S_{\nu}= & \int_{R_{\mathrm{in}}}^{R_{\mathrm{out}}} \int_{a_{\min }}^{a_{\max }} B_{\nu}\left[T_{\mathrm{d}}(a, R)\right] Q_{\mathrm{abs}}(\nu, a) N(a, R) \\
& \times \frac{\pi a^{2}}{d^{2}} 2 \pi R d a d R,
\end{aligned}
$$

where $T_{\mathrm{d}}(a, R)$ is the dust temperature as a function of particle size and orbital radius, $Q_{\mathrm{abs}}(\nu, a)$ is the absorption coefficient, and $d$ is the distance to the star. For particle sizes smaller than 3 $\mathrm{mm}$ in radius, we computed $Q_{\mathrm{abs}}(\nu, a)$ using the procedure described in Wolf \& Hillenbrand (2003) and the optical constants for "astronomical silicates" (Weingartner \& Draine 2001). For larger particles, we adopted $Q_{\mathrm{abs}}(v)=1$, which is valid for the wavelength range considered here $(\lambda \leqslant 70 \mu \mathrm{m})$. Dust temperatures were computed assuming the disk is optically thin and in thermal equilibrium with the stellar radiation field.

In Figure 16, we present calculations that illustrate how various model parameters affect the resulting spectra. The model disk surrounds a solar-type star and contains minimum grain sizes that span the range from the approximate radiation blowout size (Burns et al. 1979) to the smallest grain size that radiates like a blackbody at $70 \mu \mathrm{m}$. The inner-disk radii range from the terrestrial planet zone in our solar system to the Kuiper Belt. The models are compared to sources with IRS excesses that are confirmed by MIPS $24 \mu \mathrm{m}$ or $70 \mu \mathrm{m}$ photometry.

The main features of the data that need to be explained are the shape of the IRS spectra and the intensity of the MIPS 70 $\mu \mathrm{m}$ photometry relative to the IRS excess. The density contours allows one to assess the median behavior and the deviation around a "typical" debris disk. Models with small grains $(\lesssim 1$ $\mu \mathrm{m})$ and small inner-disk radii $(\lesssim 10 \mathrm{AU})$ tend to reproduce the general shape of the IRS spectra, but underestimate the $70 \mu \mathrm{m}$ flux densities for those stars detected at this longer wavelength due to the falloff in small grain radiative efficiency. If the minimum grain size is $\gtrsim 3 \mu \mathrm{m}$, the models can explain the level of $70 \mu \mathrm{m}$ emission, but then cannot produce the tail of warm excess emission observed between 20 and $30 \mu \mathrm{m}$. When $70 \mu \mathrm{m}$ excess emission is not detected, then a broad combination of model parameters can reproduce the observations. From these illustrative models of debris dust located over a range of orbital distances from a central star and having a range of grain sizes, we conclude that some of the features typical of the observations can be explained, but that a more systematic parameter study is needed. In the following subsections, we first examine which of the disk parameters can be constrained robustly, and then study the time evolution of the plausible debris belts.

\subsection{Constraints on the Inner Disk Radius}

To place constraints on the disk and grain properties, we used a Bayesian approach to infer the likelihood of model parameters given the observational data (see, e.g., Lay et al. 1997) for the debris disks that have reliable infrared excesses (see Section 3.6). We constructed a grid of models spanning the three-dimensional parameter space of the inner-disk radius, disk width ( $\left.\Delta R=R_{\text {out }}-R_{\text {in }}\right)$, and the power-law slope of the mass surface density. Plausible ranges for each of the parameters were considered. The inner-disk radius was varied between 0.1 and $1000 \mathrm{AU}$ to encompass the size scales inferred for protoplanetary disks. The disk width was varied between 0.1 and $1000 \mathrm{AU}$ to allow for narrow rings and wide belts. Models were computed for surface density power laws (see Equation (3)) between -1.5 to 0 in steps of 0.5 , encompassing the mass surface density of the current solar system $(\alpha=-1.5$; Weidenschilling $1977)$, of that commonly inferred for protoplanetary disks $(\alpha=-1$; Beckwith et al. 1990; Kitamura et al. 2002; Andrews $\&$ Williams 2005), and a constant surface density $(\alpha=0)$.

Spectra over the Spitzer wavelength range were computed for each combination of the above parameters assuming an optically thin disk with particle size distribution extending from the radiation blowout size to a $1000 \mathrm{~km}$ radius. The spectral types for stars with debris disks span between K3 and F5, or approximately an order of magnitude in stellar luminosity. The corresponding radiation blowout size varies between 0.2 and $0.7 \mu \mathrm{m}$ (Burns et al. 1979). Nonetheless, we did not compute a grid of models for each source, but adopted stellar parameters for a canonical solar-type star and fixed the radiation blowout size at $a=0.5 \mu \mathrm{m}$. The maximum particle radius of 1000 $\mathrm{km}$ corresponds to the size of the bodies needed to excite the collisional cascade in the debris disk models by Kenyon $\&$ Bromley (2004). The maximum particle size has negligible influence on the model calculations for the observed emission since large bodies contain a relatively small surface area for the adopted particle size distribution.

The model at a given grid point was fitted to the observations with a normalization constant as a free parameter that is proportional to the total particle cross-sectional area for optically thin emission. The photospheric contribution was determined using the fits described in Section 3. The relative probability of the model, given the data, is proportional to $e^{-\chi^{2} / 2}$, where $\chi^{2}$ is the sum of the squares of the difference between the model and the data normalized by the observational uncertainties. The probability distribution of a single model parameter is determined by summing the probabilities over the other two model parameters, and normalizing the integrated probability to unity. The parameter constraints from the ensemble of sources is then computed as the sum of the probability distributions for each source, and renormalizing the sum to unity.

The probability distributions are only meaningful if the model provides a reasonable fit to the observations, which can be judged from the minimum $\chi^{2}$ values across the grid. For model fits to the IRS spectra between 12 and $35 \mu \mathrm{m}, 35$ of the debris disks have probabilities $>0.01$ that the $\chi^{2}$ residuals are consistent with noise. For fits to both the IRS spectra and the MIPS $70 \mu \mathrm{m}$ photometry (detections and non-detections), 27 sources have probabilities $>0.01$. In general, sources with 70 $\mu \mathrm{m}$ detections have higher $\chi^{2}$ values that reflect the difficulty in finding models that can fit the IRS spectral shape and the 


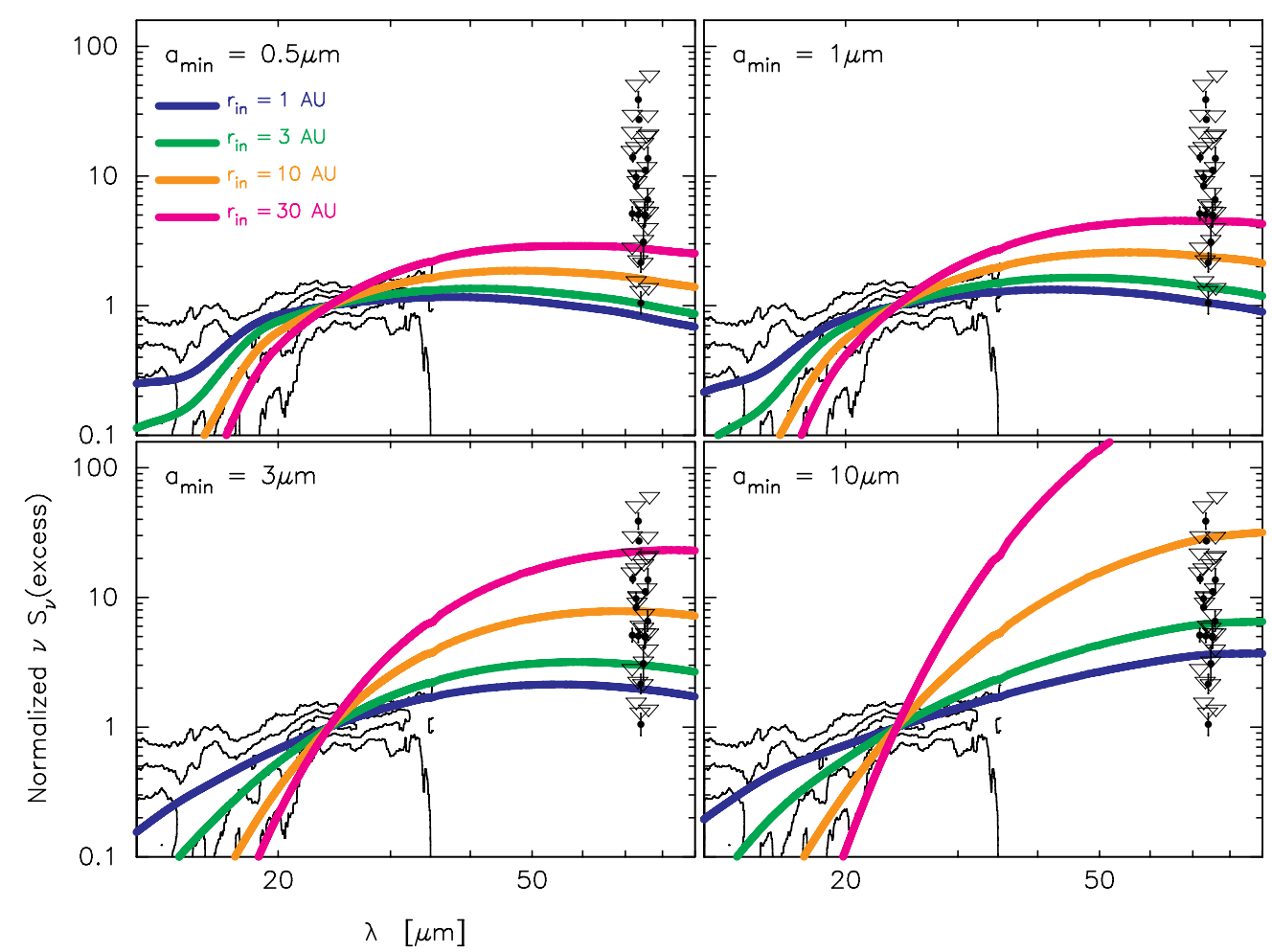

Figure 16. Comparison of dust disk models with the observed spectra for 45 debris disks with a $70 \mu \mathrm{m}$ excess or both a $24 \mu \mathrm{m}$ and IRS excess. The contours and gray scale represent the average IRS emission excess above the photosphere normalized to the $24 \mu \mathrm{m}$ excess and converted to a density plot. Contours are at $0.4,0.6$, and 0.8 of the peak density and represent the typical shape of the excess emission. MIPS $70 \mu \mathrm{m}$ detections (filled circles) and upper limits (triangles) are indicated discretely. The solid curves represent model dust emission from silicate grains for a constant-surface density disk $(\alpha=0)$ with an outer radius $R_{\text {out }}=50 \mathrm{AU}$ surrounding a solar-type star with $M_{*}=1 M_{\odot}, L_{*}=1 L_{\odot}$, and $T_{*}=5780 \mathrm{~K}$. The grain radii follow a power-law distribution $\left(N(a) \propto a^{-3.5}\right)$ with $a_{\max }=1 \mathrm{~km}$. Model calculations are shown for different minimum grain radii $a_{\min }=0.5,1,3$, and $10 \mu \mathrm{m}$ in each panel. Within a panel, the four curves indicate inner-disk radii $R_{\text {in }}=1,3,10$, and 30 AU. A small wavelength offset has been added to the 70 photometry for clarity.

$70 \mu \mathrm{m}$ photometric excess simultaneously (as illustrated in Figure 16).

Probability distributions for infrared excess sources with and without MIPS $70 \mu \mathrm{m}$ detections are presented in the top panels of Figure 17 for fits to the IRS spectra only, and in the bottom panels for fits to both the IRS spectra and 70 $\mu \mathrm{m}$ photometry. Best-fit model parameters for each source are represented by circles. We do not find any correlation of best-fit model parameters with spectral type, suggesting that adopting a single stellar model did not significantly impact the results. These histograms indicate the constraints on the model parameters for the ensemble of observed debris disks. For two of the parameters, surface density power law and disk width, the probability distributions are relatively flat. The one input parameter having moderate constraint is the inner-disk radius. This is most apparent in the probability distribution that considers only the IRS spectra (which are most diagnostic of warm inner-disk material). Most debris disks appear to have inner radii between 3 and $40 \mathrm{AU}$, with somewhat larger inner radii for stars with $70 \mu \mathrm{m}$ detections. We explore the implications of this result starting in Section 5.3.2.

For the sources with $70 \mu \mathrm{m}$ detections, adding the $70 \mu \mathrm{m}$ flux densities as a constraint in the model fits accentuates many of the trends described above (see Figure 17). Models with flatter surface density profiles are generally preferred. The inner-disk radius increases to $\sim 100-200 \mathrm{AU}$. There is a strong tendency for a large disk width; indeed, since the most probable disk width is also the maximum value in the model grid (1000 AU), these model calculations have not established the upper bound of the outer-disk radius. Disks this wide, however, are inconsistent with the other observations of debris disks around solar-type stars in that scattered-light images and resolved submillimeter images typically find radii less than 200 AU (Schneider et al. 2006; Ardila et al. 2004; Greaves et al. 1998). Furthermore, the reduced $\chi^{2}$ values for the model fits tend to be high for sources with $70 \mu \mathrm{m}$ detections, indicating as we illustrated earlier that the model has difficulty fitting both the IRS spectral shape and the $70 \mu \mathrm{m}$ photometry. Better fits with smaller outer-disk radii could be obtained by modifying the model assumptions to decrease the relative number of smaller grains. This can be accomplished, for example, by adopting for a less steep grain size distribution than $N(a) \propto a^{-3.5}$, or adopting a larger minimum grain size. Also, improved model fits could be obtained by allowing for a population of small grains that are decoupled spatially from the larger grains, as was found for the Vega debris disk (Su et al. 2006). Finally, adopting a different grain composition (e.g., water ice for the outer-disk particles), may modify the fits as well.

\subsection{Evolution of the Planetesimal Belts}

The analysis presented in Section 5.2 was intended to constrain the properties of the debris systems, which indicate the presence of planetesimal belts. The most strongly constrained parameter is the inner belt radius, which we find is typically 3-40 AU. We now extend our analysis, adopting these parameters, in order to study temporal evolution of the debris belt properties. The main observational characteristics of the 

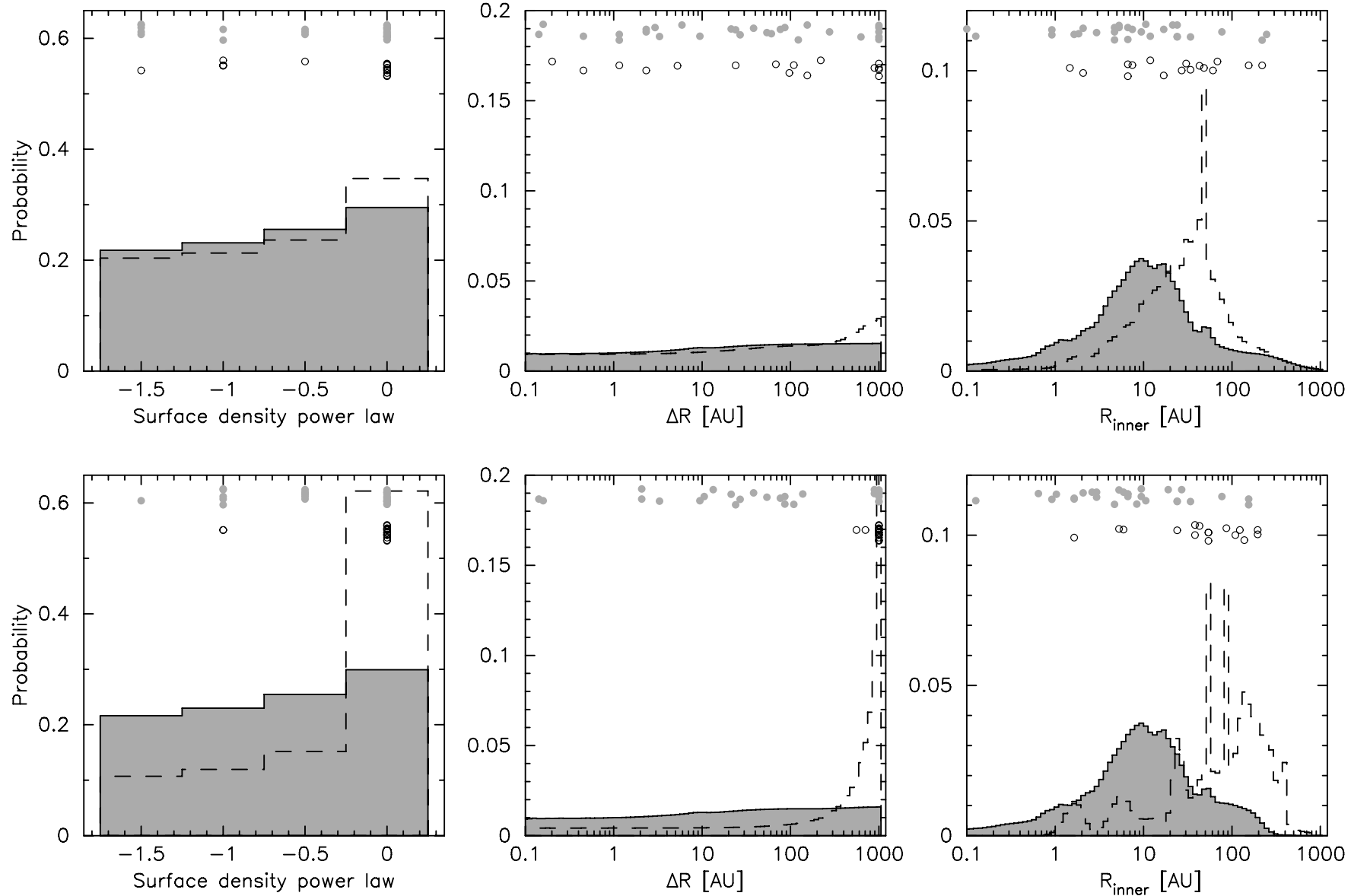

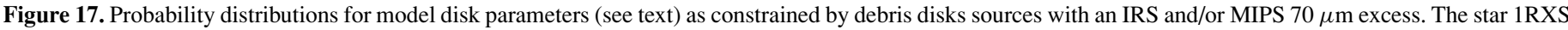

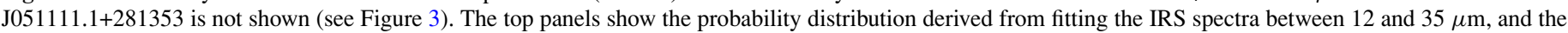

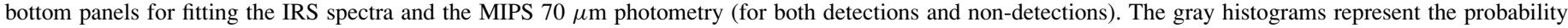

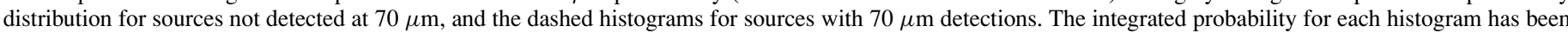
normalized to unity. The parameters for the best-fit models are indicated by the open and filled circles for sources with and without $70 \mu \mathrm{m}$ detections, respectively.

observed debris disks related to temporal evolution (Figures 11, 12,13 , and 15) are (1) the lack of debris emission at wavelengths shorter than $16 \mu \mathrm{m}$ for ages older than $3 \mathrm{Myr}$, (2) a flat distribution to the upper envelope of $24 \mu \mathrm{m}$ excesses for age $\lesssim$ $300 \mathrm{Myr}$ with a decline toward older ages, and (3) the apparent decline in the maximum $70 \mu \mathrm{m}$ excess emission for stars older than $\sim 300$ Myr. In this section, we explore if temporal collisional models can explain these aspects of the observational data.

We adopt the analytic model developed by Dominik \& Decin (2003) and extended by Wyatt et al. (2007a) to describe the collisional evolution of a planetesimal belt in quasi-steady-state equilibrium. The model posits that small grains are continually produced by collisional grinding of massive bodies, and mass is removed by radiation blowout of the smallest grains. For a system in collisional equilibrium, the mass surface density of solid particles in a narrow annulus of a planetesimal belt $(\Delta R \ll R)$ as a function of time is

$$
\Sigma(t)=\frac{\Sigma_{\mathrm{o}}}{1+t / t_{\mathrm{co}}},
$$

where $\Sigma_{\mathrm{o}}$ is the initial mass surface density of particles and $t_{\mathrm{co}}$ is the collisional timescale for the largest planetesimals when the cascade begins at $t=0$. A similar expression is valid for the number of particles and the cross-sectional surface area. In the calculations described below, we use the formula for $t_{\mathrm{co}}$ presented in Equation (13) in Wyatt et al. (2007a). However, it is instructive to view an approximate formula for $t_{\mathrm{co}}$ to understand how the collisional evolution depends on disk parameters. We make three assumptions: (1) a particle size distribution of $N(a) \propto a^{-3.5}$ (Dohnanyi 1969), (2) the orbital eccentricity $(\epsilon)$ is approximately equal to the orbital inclination, and (3) the size of the planetesimals that can destroy the largest planetesimals is much smaller than $a_{\max }$. The collisional time at $t=0$ for a narrow planetesimal belt at orbital radius $R$ is then

$$
\begin{aligned}
t_{\mathrm{co}}(R) \approx & 0.09 \mathrm{Myr} \frac{\rho}{2.7 \mathrm{~g} \mathrm{~cm}^{-3}} \frac{a_{\max }}{1000 \mathrm{~km}}\left(\frac{R}{1 \mathrm{AU}}\right)^{7 / 3} \\
& \times\left(\frac{Q_{\mathrm{D}}}{2 \times 10^{6} \mathrm{ergs} \mathrm{g}^{-1}}\right)^{5 / 6}\left(\frac{\Sigma_{\mathrm{o}}}{1 \mathrm{~g} \mathrm{~cm}^{-2}}\right)^{-1}\left(\frac{\epsilon}{0.067}\right)^{-5 / 3}
\end{aligned}
$$

where $\rho$ is the particle density and $Q_{\mathrm{D}}$ is the specific incident energy required to destroy a planetesimal (Wyatt et al. 2007a). In this model, the collisional timescale is inversely proportional to the surface density. For $t \ll t_{\mathrm{co}}$, the mass surface density of the disk at radius $R$ is constant in time since the largest particles in the system have undergone few collisions. The spread in debris disk luminosities at young ages then depends on the cross-sectional surface area at $t=0$ and the distance of the dust from the star. However, for $t \gg t_{\mathrm{co}}$, the mass surface density, and hence the emission from the disk, is independent of the 
initial disk surface density (see Dominik \& Decin 2003; Wyatt et al. 2007a).

To compute the emission from a debris disk as a function of time, the disk was divided into annuli of width $\Delta R=2 \epsilon R$ that corresponds to the range of orbital radii for a particle. As an approximation to the different evolution timescales at different radii in the disk, Equations (5) and (6) were applied to each annulus separately. The emission from individual annuli was computed using Equation (4) and then summed to get to the total disk emission. The model assumes that the collisional cascade begins at a stellar age of $0 \mathrm{Myr}$. We note that primordial disks survive for 1-10 Myr (see, e.g., Haisch et al. 2001), and the collisional cascade in the planetesimal belt will be delayed relative to the stellar age by at least the minimum time to form the planetesimals.

\subsubsection{Gas-Giant and Kuiper Belt Zones}

We first attempt to explain the excess characteristics at 8,16 , and $24 \mu \mathrm{m}$. The $70 \mu \mathrm{m}$ data are not used since the disk properties needed to produce this emission are poorly constrained by the data (see Section 5.2 and Figure 17). The model planetesimal belt has a fiducial inner radius of $10 \mathrm{AU}$ in accordance with the disk properties inferred in Section 5.2. In the following section, we relax this assumption and explore the signatures of planetesimals in the inner $10 \mathrm{AU}$. For the other disk parameters we explore a range of properties. The disk surface density at $t=0$ is parameterized as a constant surface density disk of $\Sigma(R)=\Sigma_{\mathrm{o}}$; models were computed for $\Sigma_{\mathrm{o}}$ ranging from 0.03 to $3 \mathrm{~g} \mathrm{~cm}^{-2}$. We consider disks with an outer radius of 15 and $100 \mathrm{AU}$ to emulate a narrow ring and a wide belt, respectively. Extending the disk beyond $100 \mathrm{AU}$ will change the $24 \mu \mathrm{m}$ flux density by less than $10 \%$ since the dust at these radii is too cool to radiate prominently at $\lambda<24 \mu \mathrm{m}$.

In Figure 18, we present model calculations for the temporal evolution of the 8,16 , and $24 \mu \mathrm{m}$ emission. In the top panels, different colored curves represent distinct values of $\Sigma_{0}$ for an outer radius of $15 \mathrm{AU}$ (solid curves) and $100 \mathrm{AU}$ (dashed curves). The IRAC $8 \mu \mathrm{m}$, IRS $16 \mu \mathrm{m}$, and MIPS $24 \mu \mathrm{m}$ observations discussed in Section 3 are also shown, with black circles representing sources with infrared excesses at the wavelength.

The model curves shown in the top row of panels of Figure 18 shows a flat emission profiles for younger ages, followed by a $t^{-1}$ fall off in the intensity toward older ages. This general shape is expected based on the adopted density distribution with time (Equation (5)). Models with an outer radius of $100 \mathrm{AU}$ have brighter emission than models with a $15 \mathrm{AU}$ outer radius since the larger disk contains more dust surface area.

The models predict that the $8 \mu \mathrm{m}$ excess is $<0.7 \%$ at an age of $1 \mathrm{Myr}$ for all surface densities considered here. This excess level is consistent with the observed $3 \sigma$ upper limit of $3 \%$ found toward individual stars. The lack of $8 \mu \mathrm{m}$ emission in the models is expected since the inner $10 \mathrm{AU}$ was assumed to be evacuated of dust.

At 16 and $24 \mu \mathrm{m}$, the models cannot provide a unique interpretation of the results since the disk size and powerlaw surface density are degenerate parameters. Nonetheless, we show in Figure 18 that a class of models exists that can account for many of the observed characteristics. For example, these models predict $16 \mu \mathrm{m}$ emission of less than $20 \%$ for surface densities $\lesssim 0.3 \mathrm{~g} \mathrm{~cm}^{-2}$. At $24 \mu \mathrm{m}$, the emission is relatively constant for ages $\lesssim 100 \mathrm{Myr}$ if $\Sigma_{\mathrm{o}}<0.1 \mathrm{~g} \mathrm{~cm}^{-2}$ and $R_{\text {out }}=100 \mathrm{AU}$, or for $\Sigma_{\mathrm{o}}<0.3 \mathrm{~g} \mathrm{~cm}^{-2}$ and $R_{\text {out }}=15 \mathrm{AU}$. The $R_{\text {out }}=15$ AU models agrees well with the observations in that the emission falls sharply with age at $\sim 300$ Myr. However, for models with $R_{\text {out }}=100 \mathrm{AU}$, the $24 \mu \mathrm{m}$ emission persists for as long as $10 \mathrm{Gyr}$, which is much longer than observed. The time constant of the emission could be decreased for the $R_{\text {out }}=100$ AU models to better match the data by decreasing the maximum planetesimal size, having a steeper radial density profile, or increasing the particle eccentricity.

While we have not done an exhaustive parameter study, we conclude that basic characteristics of the 8,16 , and $24 \mu \mathrm{m}$ excess emission can be explained by a planetesimal belt with an inner radius of $10 \mathrm{AU}$ in quasi-steady-state collisional evolution. This model can account for the magnitude of the excesses, and the relatively flat distribution of $24 \mu \mathrm{m}$ excess for young ages, and the decline in the $24 \mu \mathrm{m}$ excess for ages older than $\sim$ 100-300 Myr.

\subsubsection{Depletion of the Inner Disk}

The models presented in the top panel of Figure 18 assume an inner-disk radius of $10 \mathrm{AU}$, which is a typical radius inferred from analysis of the IRS spectra (Section 5.2). By contrast, the inferred inner-disk radius in primordial disks is $\lesssim 0.3 \mathrm{AU}$ (e.g., Akeson et al. 2005), and at least in our solar system, the terrestrial planets must have formed from planetesimals well within 3 AU. Collisions in the inner planetesimal belt would presumably produce warm debris that will emit at wavelengths shorter than $24 \mu \mathrm{m}$ and could be traced by IRS spectra and IRAC photometry.

One difficulty in detecting warm debris disks is related to the speed at which they are expected to evolve. For a disk with a power-law surface density distribution (Equation (3)), the timescale for collisional growth of planetesimals varies with orbital radius as $R^{3 / 2-\alpha}$ (Lissauer 1987). ${ }^{17}$ We thus expect that the collisional cascade will also proceed on faster timescales at smaller orbital radii for $\alpha<1.5$, and that the inner disk will be depleted more quickly of dust mass via radiation blowout of the smallest grains than the outer disk. We consider then if a planetesimal belt that originally extended to small radii will deplete on timescales fast enough to remain consistent with the lack of observed excess emission at 8 and $16 \mu \mathrm{m}$, or if another mechanism is needed to increase the dissipation timescale. For this exercise, we consider a disk model with an inner radius of $0.5 \mathrm{AU}$, and otherwise adopt the disk parameters assumed in Section 5.3.1. Model calculations that show the temporal variations of the emission are presented in the bottom row of panels in Figure 18.

As expected, changing the inner-disk radius from $10 \mathrm{AU}$ to $0.5 \mathrm{AU}$ increases the amount of short-wavelength emission. For $t \lesssim 3 \mathrm{Myr}$, the model $8 \mu \mathrm{m}$ disk emission is $\geqslant 3.0 \%$ above the photosphere and detectable in individual sources with the FEPS IRAC observations in disk models with $\Sigma_{\mathrm{o}} \geqslant 3 \mathrm{gm} \mathrm{cm}^{-2}$. These models are consistent with the observations in that no optically thin $8 \mu \mathrm{m}$ emission was detected in FEPS sample, which covers stellar ages older than 3 Myr.

Based on the FEPS observations, 12\% of stars are surrounded by optically thick disks between ages of 3 and $10 \mathrm{Myr}$. If we assume that the collisional cascade begins primarily after the cessation of accretion and all optically thick disks pass through a debris phase, then $12 \%$ of stars with ages of 3-10 Myr may contain recent production of $8 \mu \mathrm{m}$ debris emission.

\footnotetext{
17 The radial exponent for planetesimal growth adopted here differs from that for planetesimal destruction (see Equation (6)) because of additional assumptions about how physical parameters vary with radius in the latter model (see Wyatt et al. 2007a).
} 


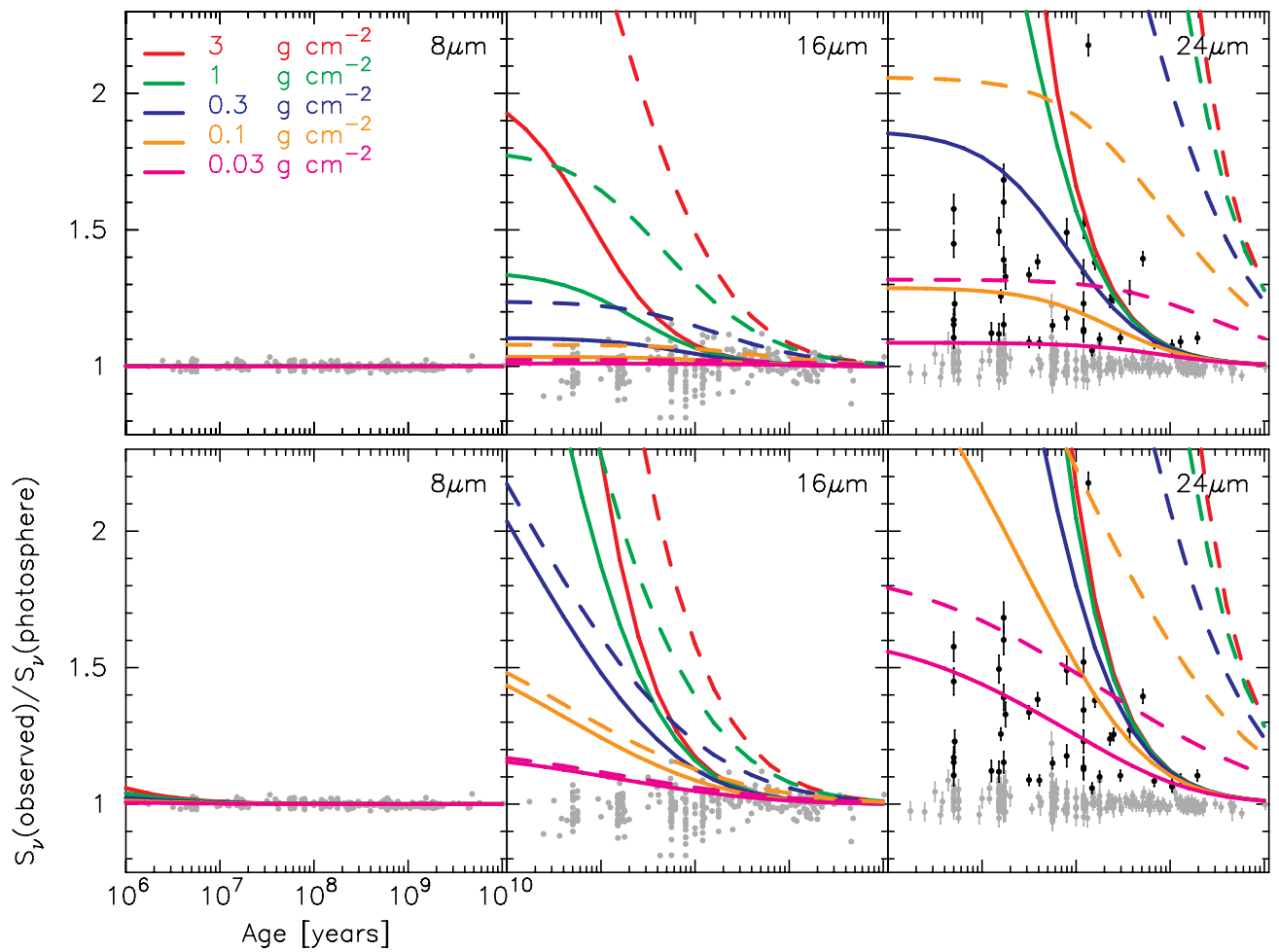

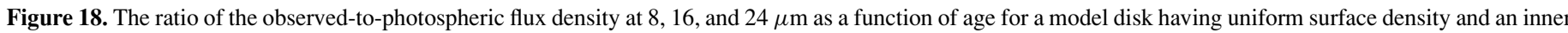

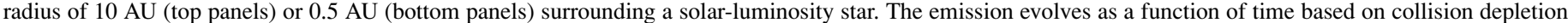

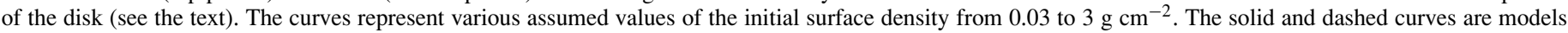

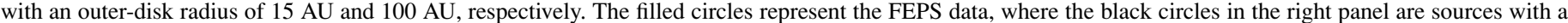
$24 \mu \mathrm{m}$ excess from a debris disk. Five sources with optically thick disks are not shown.

Since $8 \mu \mathrm{m}$ emission will be detectable for up to $3 \mathrm{Myr}$ after the start of the collisional cascade for an inner-disk radius of $0.5 \mathrm{AU}$, we expect to detect $\sim 1-2$ stars with optically thin $8 \mu \mathrm{m}$ emission. This is consistent with the null detections of optically thin emission in the FEPS sample. We conclude that the absence of $8 \mu \mathrm{m}$ detections in the FEPS sample does not meaningfully constrain the presence or lack thereof of the initial planetesimal belts at a radius of $\sim 0.5 \mathrm{AU}$, and that observations for a much larger sample of young stars are needed to probe this rapid evolutionary stage (see Currie et al. 2007).

The IRS and MIPS $24 \mu \mathrm{m}$ observations provide more meaningful constraints on the temporal evolution of the inner planetesimal belts. With the smaller inner-disk radius, the collisional timescale is smaller for the $R_{\text {in }}=0.5 \mathrm{AU}$ model than the 10 AU model considered in Section 5.3.1 for the same surface density (see Equation (6)). The emission curves are largely on the $t^{-1}$ evolution phase and the predicted 16 and 24 $\mu \mathrm{m}$ emission decline rapidly with time for most of the surfaced densities considered here (see Figure 18). Yet the observed emission is much less than predicted by many of the models explored here.

Because the inner-disk radius is smaller than considered in Section 5.3.1, this class of models requires lower overall surface densities to match the observations. Model with surface densities of $\Sigma_{\mathrm{o}}>0.03 \mathrm{~g} \mathrm{~cm}^{-2}$ produce higher 16 and $24 \mu \mathrm{m}$ flux densities and a sharp decline in the flux densities with increasing age that is inconsistent with the observations. Qualitative agreement between the models and the observations is found for $\Sigma_{\mathrm{o}}<0.03$ $\mathrm{g} \mathrm{cm}^{-2}$ in that the $16 \mu \mathrm{m}$ excess is less than $20 \%$, and that the magnitude of $24 \mu \mathrm{m}$ is consistent with the observations.
As was found in Section 5.3.1, models with $R_{\text {out }}=15 \mathrm{AU}$ provide a better match to the temporal decline than models with $R_{\text {out }}=100 \mathrm{AU}$ for the assumed parameters. We conclude that if the inner planetesimal belt initially extended to $0.5 \mathrm{AU}$, the belt can deplete fast enough to explain the observations. However, bright $16 \mu \mathrm{m}$ excess emission from debris dust would be expected to found around young ( $<3$ Myr) stars.

\subsection{Timescale of the Debris Phase}

The fraction of stars in the FEPS sample with $24 \mu \mathrm{m}$ excesses greater than $10.2 \%$ above the photosphere is constant to within the uncertainties at $\sim 15 \%$ for ages less than 300 Myr. By contrast, while some primordial disks dissipate within $1 \mathrm{Myr}$ (Padgett et al. 2006; Cieza et al. 2007), about half of solar mass stars at ages of 1-3 Myr remain surrounded by primordial disks whether traced by emission at 3-8 $\mu \mathrm{m}$ (Haisch et al. 2001; Hernández et al. 2007), $10 \mu \mathrm{m}$ (Mamajek et al. 2004), or 24 $\mu \mathrm{m}$ (e.g., Lada et al. 2006; Damjanov et al. 2007). We consider then why the fraction of 1 Myr stars with primordial disks, and therefore have at least the potential to form planets, is much higher than the stars detected with debris dust.

As discussed in Meyer et al. (2008), the observed fraction of stars with $24 \mu \mathrm{m}$ excesses as a function of age (see Figure 11) may reflect one of the two scenarios. One possibility is that only $\sim 15 \%$ of disks around solar-type stars form planets or large planetesimals, and the resulting debris disks emit at $24 \mu \mathrm{m}$ for $\sim 100-300$ Myr. The results presented in Section 5.3.1 indicate that a quasi-steady-state collisional cascade can explain basic characteristics of the excess emission observed in debris 
disks under certain assumptions (see also Dominik \& Decin 2003; Wyatt et al. 2007a), although this model is not a unique interpretation of the data. Another possibility is that the debris phase is more common, but for any given star it is short lived and so for any given age interval, only $15 \%$ of stars exhibit the debris phenomenon. In this interpretation, more than $60 \%$ of FEPS stars pass through a debris phase at some point in their lifetime, suggesting that most solar-type stars form planetary systems.

The critical distinction between the above two scenarios is the lifetime of the debris phase and whether the dust is continuously replenished over tens of million of years in a quasi-steady state, or if the dust is a transient phenomenon. We reconsider the arguments in Meyer et al. (2008) using the full complement of Spitzer data from the FEPS program. We assume that the planetesimal belt extends from inner radius $R_{\text {in }}$ to outer radius $R_{\text {out }}=R_{\text {in }}+\Delta R$. The lower limit to the debris disk lifetime is set by the timescale for the collisional cascade to propagate through the disk. For a power-law radial surface density of $\Sigma(R) \propto \Sigma_{0} R^{\alpha}$, the timescale for collisional growth is proportional to $R_{\text {in }}^{3 / 2-\alpha} \Sigma_{\mathrm{o}}^{-1}$. If the debris phase is a transient phenomenon, the duration of the debris phase, defined as $\Delta t$, is much less than the age intervals used to create Figure 12, or $t / \Delta t<3$. The width of the planetesimal belt $(\Delta R)$ to limit the debris phase to $\Delta t$ is then given by $\Delta R \ll 2(1.5-\alpha)^{-1} R_{\text {in. }}$. For a typical inner radius of $\sim 10 \mathrm{AU}$ and $\alpha$ between 0 and 1.5, the width of the planetesimal belts responsible for the $24 \mu \mathrm{m}$ emission must be $\ll 7-13$ AU to allow for a short debris phase.

We consider the likelihood that most of the debris disks have narrow planetesimal belts given the observations. The model calculations presented in Section 5.2 suggest that the grains producing the $70 \mu \mathrm{m}$ emission, and by inference the corresponding planetesimal belt, extend over tens of astronomical units. By the above arguments, we expect the debris phase for these systems to persist over tens of millions of years. Whether or not the emission is detectable at 24 or $70 \mu \mathrm{m}$ over this time period depends on the characteristics of the planetesimal belt.

As demonstrated in Section 5.3.1, large planetesimal belts in a quasi-steady state can produce $24 \mu \mathrm{m}$ emission above the FEPS detection limit for $\sim 100 \mathrm{Myr}$. This result is model dependent, however, and we consider the case where $24 \mu \mathrm{m}$ emission is only produced when a "wave" of debris production propagates through the disk (Kenyon \& Bromley 2002, 2005). In this scenario, stars have narrow planetesimal belts at various radii, and we observe $24 \mu \mathrm{m}$ emission only when the collisional cascade is initiated at a given radius. The expectation is that debris in planetesimal belts at large radii will be produced at later times, such that the observed 100-300 Myr lifetime of the $24 \mu \mathrm{m}$ emission would translate to a factor of 6-10 variation in radii for $\alpha=-1$. The temperature of the debris emission would decrease by a factor of $\sim 2.5-3$ over this range of radii for blackbody grains. While the observed range of dust temperatures is of this magnitude (see Figure 14), there is no evidence for a systematic change in the dust temperatures with age to support this scenario.

Alternatively, the 100-300 Myr lifetime of strong $24 \mu \mathrm{m}$ emission could primarily reflect variations in the disk surface density, where the collisional cascade is initiated at later times in lower density disks. Since $24 \mu \mathrm{m}$ excesses are observed over a factor of 10-30 in age, we expect a corresponding variation in the magnitude of the $24 \mu \mathrm{m}$ excess, with smaller excesses at older ages. As shown in Figure 11, there is no evidence for a systematic decline of this magnitude for stars younger than $300 \mathrm{Myr}$.
Another possibility is that the debris emission is produced by catastrophic events that produce copious amounts of debris dusts for brief periods (Rieke et al. 2005). Wyatt et al. (2007b) evaluated the probability of such collisions and found that the frequency of such collisions will vary with time as $t^{-2}$ at a given orbital radius. Assuming the orbital radii of planetesimal belts are approximately the same, one would expect a two order of magnitude decrease in the fraction of disks exhibiting a catastrophic collision between 10 and 100 Myr. This contrasts with the observed flat distribution of fractional excesses with age for ages $\lesssim 300 \mathrm{Myr}$ (see Figure 12). We therefore suggest that the $15 \%$ detection rate of debris disks younger than $300 \mathrm{Myr}$ primarily reflects that only $\sim 15 \%$ of solar-type stars exhibit 24 $\mu \mathrm{m}$ excesses (greater than $10.2 \%$ above the photosphere) over their lifetime.

If the fraction of stars that pass through a relatively long-lived, luminous $24 \mu \mathrm{m}$ debris phase is about $15 \%$, we return now to the question of why this percentage is so low given that most young stars are surrounded by primordial disks. The spread in lifetimes of primordial disks is $\lesssim 10$ Myr. If planetesimal production from primordial disks is the same regardless of the lifetime of the primordial phase, then a delay of $10 \mathrm{Myr}$ in the offset of debris production will have little influence on the duration ( $\sim 100-300 \mathrm{Myr}$ ) of the $24 \mu \mathrm{m}$ debris phase. However, we can anticipate a range of planetesimal growth timescales related to the observed dispersion in primordial disk masses, as well as diversity in dynamical processes that sculpt planetary systems.

It is tempting to compare this frequency of detected debris $(>15 \%)$ to the occurrence of gas-giant planets. Cumming et al. (2008) report the frequency of Sun-like stars with gas giants $>0.1 M_{\text {Jupiter }}$ of up to $20 \%$ when extrapolated out to $20 \mathrm{AU}$. Moro-Martín et al. (2007a) were unable to confirm a correlation between debris disk phenomenon and the presence of close in gas-giant planets (see however Moro-Martín et al. 2007b, for a notable example). Apai et al. (2008) did not find evidence of massive gas giants in debris systems with large inner holes. It remains unclear to whether these debris disks represent those with inner gas giants or are another population representing a diverse planetary system architecture. Perhaps these large inner holes are evidence of rapid planet formation in the terrestrial planet zone. The question remains whether those sources lacking obvious debris represent dynamically full planetary systems that rapidly cleared all potential sources of debris, or primordial disks that dissipated leaving nothing behind.

Could we have missed a significant number of debris disks in our survey? Debris disks could not have been detected if the dust is colder or less luminous than the sample detected here. In the context of the collisional planetesimal model, on timescales shorter than the collisional timescale, the most massive and larger planetesimal belts will produce more luminous $24 \mu \mathrm{m}$ emission. Similarly, increasing the inner-disk radius would decrease the $24 \mu \mathrm{m}$ disk emission. Evidence for additional debris disks is present within the FEPS data. Analysis of the IRS spectra suggests a possible additional 21 disk candidates that do not exhibit a $24 \mu \mathrm{m}$ excess, and most of the sources appear younger than $300 \mathrm{Myr}$. If these infrared excesses are real, the debris disk percentage would increase from $\sim 15 \%$ to $24 \%$. However, this is still much less than the primordial disk fraction found around stars, and it remains unclear what primordial disk properties produced this small percentage of debris disk detections. 


\section{COMPARISON TO THE SOLAR SYSTEM}

The basic geometry of the planetesimal disks inferred from debris dust surrounding the FEPS sample is a cleared inner disk out to $\sim 10$ AU with optically thin dust beyond these radii. For the sources with $70 \mu \mathrm{m}$ detections, the inferred radii are many tens of AU wide (see also Hillenbrand et al. 2008). This morphology is similar to that of the Kuiper Belt of our solar system. In this section, we make more quantitative comparison between the solar system and the debris disks in the FEPS sample, considering both Kuiper-Belt-like dust on tens of AU scales and the available constraints on zodiacal dust on several AU scales.

The model results in Section 5 suggest that the initial surface density of solids for FEPS detected debris disks is $\lesssim 3 \mathrm{~g} \mathrm{~cm}^{-2}$ at $1 \mathrm{AU}$; this is less than surface density of solids expected for the "minimum-mass-solar-nebula" ( $\sim 7-$ $30 \mathrm{~g} \mathrm{~cm}^{-2}$; Weidenschilling 1977; Hayashi 1981). However, the model surface densities are extremely sensitive to the adopted parameters since most of the surface area is contained in smaller particles, while the large particles contain the majority of the mass. For example, by changing the power law slope of the size distribution from -3.5 to -3.2 for particle sizes between $0.5 \mu \mathrm{m}$ and $1000 \mathrm{~km}$, the total cross-sectional surface density of particles can be maintained if the mass surface density is increased by a factor of 1000 . Given the poor constraints on the planetesimal belt mass, our comparisons to the solar system will focus on the fractional dust luminosity which is more closely tied to the observations.

We first consider the sensitivity of the Spitzer observations to the present-day solar system zodiacal dust (see also Mamajek et al. 2004). Following Gaidos (1999), the luminosity emitted by zodiacal dust is $L_{\text {zodi }}=8 \times 10^{-8} L_{\odot}$ with a characteristic temperature of $260 \mathrm{~K}$ (Reach et al. 1996). The implied surface area of particles to reproduce this luminosity given the characteristic temperature is $\sim 10^{21} \mathrm{~cm}^{2}$, which Gaidos (1999) defined as 1

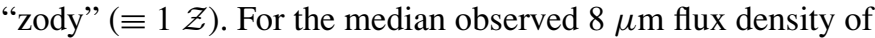
$127 \mathrm{mJy}$, and the $3 \sigma$ sensitivity limit of $3 \%$ to $8 \mu \mathrm{m}$ excesses (see Section 3.1), the IRAC observations can detect $8 \mu \mathrm{m}$ excesses of $\sim 4$ mJy typically. At the median distance of $50 \mathrm{pc}$ to stars in the FEPS sample, this limit corresponds to a sensitivity limit of $\sim 4500 \mathcal{Z}$ for $260 \mathrm{~K}$ dust. Similarly, the synthetic IRS $16 \mu \mathrm{m}$ photometry is sensitive to an infrared excess of $16.2 \%$ (5 mJy for the typical star), and 5.4\%-10.2\% (0.8-1.6 mJy) for MIPS $24 \mu \mathrm{m}$. For $260 \mathrm{~K}$ dust, these limits translate to a sensitivity limit of $1400 \mathcal{Z}$ and $220-440 \mathcal{Z}$, respectively. Our sensitivity limits then do not preclude the presence of zodiacal-type dust present in the FEPS debris disk sample.

The MIPS $70 \mu \mathrm{m}$ observations are sensitive to cooler temperature dust $(\sim 70 \mathrm{~K})$, and the more appropriate comparison is the Kuiper Belt. Emission from debris dust in the Kuiper Belt has not been detected. Stern (1996) place an upper limit of $3 \times 10^{-6}$ to the optical depth at $60 \mu \mathrm{m}$, and models of the Kuiper Belt population suggest that the current luminosity emitted by dust in the Kuiper Belt is $\sim 10^{-7} L_{\odot}$ (Backman et al. 1995). This disk luminosity is substantially lower than the luminosities derived for disks in the FEPS sample. However, the Sun, at an age of 4.57 Gyr (Bahcall et al. 1995), is significantly older than most stars observed here ( $\lesssim 3$ Gyr). Therefore we must account for temporal depletion of small dust grains from Poynting-Robertson drag and collisional processes to make a proper comparison between the FEPS sample and the Kuiper Belt (e.g., Meyer et al. 2007). Including the effects of the dynamical rearrangements of the outer planets indicated in the Nice Model (e.g., Gomes et al. 2005 ) is vital for a complete comparison to the solar system, but is beyond the scope of this work.

We use the quasi-steady-state collisional model developed by Dominik \& Decin (2003) and described in Section 5 to project forward the evolution of the FEPS debris disks. We can identify three regimes for the anticipated evolution of the disks. When the age of the debris disks is shorter than the initial collisional timescale $\left(t_{\mathrm{co}}\right)$ of the largest particles in the cascade, the debris emission will be approximately constant in time. When system ages are $>t_{\mathrm{co}}$, but collisions still dominate, the debris luminosity will vary in time as $t^{-1}$. Finally, when the Poynting-Robertson (PR) drag timescale becomes shorter than the collisional timescale, the number of particles and the emission will vary in time as $t^{-2}$.

For the debris disks detected by FEPS, the collisional timescale is likely shorter than the PR timescale for all particle sizes (Dominik \& Decin 2003; Hillenbrand et al. 2008). However, it is not possible to determine if the debris disk luminosity is on the constant, $t^{0}$, or the $t^{-1}$ phase of collisional evolution. We assume that each debris disk is currently in the $t^{-1}$ phase to achieve the fastest dissipation time. The debris system luminosity evolves as $t^{-1}$ until the Poynting-Robertson drag is shorter than the instantaneous collisional timescale. From Backman \& Paresce (1993) the collisional timescale is

$$
t_{\mathrm{c}}=\left(\frac{R}{\mathrm{AU}}\right)^{1.5} \sqrt{\frac{M_{*}}{\mathrm{M}_{\odot}}}(8 \sigma(R))^{-1} \mathrm{yr},
$$

where $\sigma(R)$ is the fraction of the geometric surface area that is covered with particles. For a uniform surface density disk, $\sigma(R)=2 f / \ln \left(R_{\text {out }} / R_{\text {in }}\right)$, where $f$ is the fractional dust luminosity (Backman 2004). Also from Backman \& Paresce (1993) the Poynting-Robertson timescale is given by

$$
t_{\mathrm{PR}}=1900 \frac{\rho}{2.7 \mathrm{~g} \mathrm{~cm}^{-3}} \frac{a}{\mu \mathrm{m}}\left(\frac{R}{\mathrm{AU}}\right)^{2}\left(\frac{L_{*}}{\mathrm{~L}_{\odot}}\right)^{-1} \mathrm{yr},
$$

where $a$ is the particle size. Adopting an inner-disk radius of $10 \mathrm{AU}$ and an outer radius of $100 \mathrm{AU}$, the PR timescale for the smallest grains $(0.5 \mu \mathrm{m})$ will be shorter than the collisional timescale for $f<6 \times 10^{-6}$.

The debris disks detected by FEPS at ages of 20-200 Myr have fractional luminosities between $2 \times 10^{-4}$ and $3 \times 10^{-3}$. If these luminosities are projected forward in time using the above prescription for a disk that extends between 10 and $100 \mathrm{AU}$, by the age of the solar system the expected luminosity is between $3 \times 10^{-8}$ and $10^{-4}$ (see Figure 15). Between ages of 400 and 1200 Myr the median fractional luminosity observed for the FEPS detected debris disks is $2 \times 10^{-4}$. These systems project forward to luminosities of $\sim 2 \times 10^{-5}$ at 4.5 Gyr. Clearly any such projections are extremely uncertain, though we conclude that with the possible exception of the youngest debris disks, the projected luminosities are 1-2 orders of magnitude brighter than the estimated luminosity of the Kuiper Belt. These data are consistent with the solar system debris luminosity being "typical" (see Bryden et al. 2006).

\section{SUMMARY}

We have completed a Spitzer photometric (3.6, 4.5, 8, 24, and $70 \mu \mathrm{m})$ and spectroscopic $(7-35 \mu \mathrm{m})$ survey of 314 FGK solartype stars that span ages between $\sim 3 \mathrm{Myr}$ and $3 \mathrm{Gyr}$. These data were used to identify sources that have infrared emission above 
the stellar photosphere that is diagnostic of circumstellar dust. This study complements previous Spitzer studies which have surveyed either more massive A- and F-type stars (Rieke et al. 2005; Su et al. 2006), lower-mass M-type stars (Gautier et al. 2007), solar-mass field stars (Trilling et al. 2008; Beichman et al. 2006b; Bryden et al. 2006), or stars in open clusters of welldefined ages (Siegler et al. 2007; Gorlova et al. 2007; Cieza et al. 2008; Currie et al. 2008). Moreover, the extensive photometric and spectroscopic coverage enables a thorough investigation of the circumstellar dust properties.

The multiwavelength data set was utilized to select a reliable sample of sources with infrared excesses. Five sources display infrared excesses in the IRAC, MIPS, and IRS data, and have characteristics of optically thick, gas-rich, primordial disks. The other sources identified with infrared excesses have properties more akin to debris disks in that the excess emission is detected at wavelengths longer than $16 \mu \mathrm{m}$ and the fractional luminosity is $L_{\mathrm{IR}} / L_{*}<10^{-3}$. Physical properties of the putative planetesimal belts that produce the debris were inferred by fitting the spectral energy distribution emitted from an optically thin debris disk containing a power-law distribution of silicate particles $\left(N(a) \propto a^{-3.5}\right)$. The results suggest that the inner disk is typically cleared out to 3-40 AU, and for sources with detected $70 \mu \mathrm{m}$ excesses, the debris extends over tens of astronomical units.

An average $15 \%$ of the stars younger than 300 Myr have a $24 \mu \mathrm{m}$ excess more than $10.2 \%$ above the photosphere, and this fraction declines to $2 \%$ for older stars. The maximum $70 \mu \mathrm{m}$ excess exhibits decline over the same age range. The temporal properties were modeled with a planetesimal belt in quasi-steady-state collisional equilibrium where the mass is removed from the system by radiation blowout of the smallest particles. Such a model can account for the lack of excess emission shortward of $16 \mu \mathrm{m}$ and the relatively flat distribution of $24 \mu \mathrm{m}$ excesses with age. These results suggest it is not necessary to invoke transient collisional events to explain the emission characteristics though they cannot be ruled out. Another possibility is that they were cleared out to $10 \mathrm{AU}$ by dynamical processes associated with inner planet formation. We can only speculate whether those sources lacking evidence for debris in the present survey will never (or have never) exhibited such evidence, or whether they represent systems with the maximum (or minimum) numbers of planets expected from primordial disks.

The properties of the debris disks in the FEPS sample were compared to that of the solar system zodiacal dust and Kuiper Belt. The FEPS observations are sensitive to $>220$ times the luminosity of the zodiacal disk and cannot rule out the presence of a solar-system-type debris disk. The luminosity of the debris systems was compared with a simple model for the evolution of the debris dust that neglects dynamical events suggested in the Nice model of the solar system (Gomes et al. 2005). Comparing the expected evolution of the younger, luminous debris disks detected in our survey, as well as our upper limits, we cannot rule out that cold outer debris disks comparable to our own solar system are the rule rather than the exception.

J.M.C. thanks Dave Frayer and the Spitzer Science Center staff for patiently answering numerous questions regarding Spitzer data. We are grateful to the anonymous referee for their extensive comments on the paper. We would also like to thank all members of the FEPS team for their contributions over the years. This work is based on observations made with the Spitzer Space Telescope, which is operated by JPL/Caltech under a contract with NASA. The program made use of data and resources from the FEPS project, which receives support from NASA contracts 1224768,1224634 , and 1224566 administered through JPL. This research made use of the SIMBAD database, operated at CDS, Strasbourg, France, and data products from the 2MASS, which is a joint project of the U. Massachusetts and the Infrared Processing and Analysis Center/Caltech, funded by NASA and the NSF. S.W. was supported by the German Research Foundation (DFG) through the Emmy Noether grant WO $857 / 2$. M.R.M. is grateful for support from the NASA Astrobiology Institute.

\section{REFERENCES}

Akeson, R. L., et al. 2005, ApJ, 635, 1173

Andrews, S. M., \& Williams, J. P. 2005, ApJ, 631, 1134

Apai, D., et al. 2008, ApJ, 672, 1196

Ardila, D. R., et al. 2004, ApJ, 617, L147

Aumann, H. H. 1985, PASP, 97, 885

Aumann, H. H., \& Probst, R. G. 1991, ApJ, 368, 264

Aumann, H. H., et al. 1984, ApJ, 278, L23

Backman, D. E. 2004, in ASP Conf. Ser. 324, Debris Disks and the Formation of Planets: A Symposium in Memory of Fred Gillett (San Francisco, CA: ASP), 9

Backman, D. E., Dasgupta, A., \& Stencel, R. E. 1995, ApJ, 450, L35

Backman, D. E., \& Gillett, F. C. 1987, in Cool Stars, Stellar Systems and the Sun, ed. J. L. Linsky, R. E. Stencel (Berlin: Springer), 340

Backman, D. E., \& Paresce, F. 1993, in Protostars and Planets III, ed. E. H. Levy \& K. L. Lunine (Tucson, AZ: Univ. Arizona Press), 1253

Bahcall, J. N., Pinsonneault, M. H., \& Wasserburg, G. J. 1995, Reviews of Modern Physics, 67, 781

Beckwith, S. V. W., Sargent, A. I., Chini, R. S., \& Guesten, R. 1990, AJ, 99, 924

Beichman, et al. 2005a, ApJ, 626, 1061

Beichman, et al. 2005b, ApJ, 622, 1160

Beichman, et al. 2006a, ApJ, 652, 1674

Beichman, et al. 2006b, ApJ, 639, 1166

Bouwman, J., et al. 2008, ApJ, 683, 479

Bryden, G., et al. 2006, ApJ, 636, 1098

Burns, J. A., Lamy, P. L., \& Soter, S. 1979, Icarus, 40, 1

Campo Bagatin, A., Cellinio, A., Davis, D. R., Farinella, P., \& Paolicchi, P. 1994, Planet. Space Sci., 42, 1079

Carpenter, J. M., et al. 2008, ApJS, 179, 423 (Paper I)

Chen, C. H., Jura, M., Gordon, K. D., \& Blaylock, M. 2005, ApJ, 623, 493

Cieza, L. A., Cochran, W. D., \& Augereau, J.-C. 2008, ApJ, 679, 720

Cieza, L., et al. 2007, ApJ, 667, 308

Cumming, A., Butler, R. P., Marcy, G. W., Vogt, S., Wright, J. T., \& Fischer, D. A. 2008, PASP, 120, 531

Currie, T., Plavchan, P., \& Kenyon, S. J. 2008, arXiv:0807.2056

Currie, T., et al. 2007, ApJ, 659, 599

Currie, T., et al. 2008, ApJ, 672, 574

Cutri, R. M., et al. 2003, The IRSA 2MASS All-Sky Point Source Catalog (Pasadena: IPAC) http://irsa.ipac.caltech.edu/applications/Gator

Damjanov, I., Jayawardhana, R., Scholz, A., Ahmic, M., Nguyen, D. C., Brandeker, A., \& van Kerkwijk, M. H. 2007, ApJ, 670, 1337

Decin, G., Dominik, C., Waters, L. B. F. M., \& Waelkens, C. 2003, ApJ, 598, 636

Dohnanyi, J. W. 1969, J. Geophys. Res., 74, 2531

Dominik, C., \& Decin, G. 2003, ApJ, 598, 626

Gaidos, E. J. 1999, ApJ, 510, L131

Gautier, T. N. III, et al. 2007, ApJ, 667, 527

Gehrels, N. 1986, ApJ, 303, 336

Gomes, R., Levison, H. F., Tsiganis, K., \& Morbidelli, A. 2005, Nature, 435, 466

Gorlova, N., Balog, Z., Rieke, G. H., Muzerolle, J., Su, K. Y. L., Ivanov, V. D., \& Young, E. T. 2007, ApJ, 670, 516

Greaves, J. S., et al. 1998, ApJ, 506, L133

Greenberg, R., Wacker, J. F., Hartmann, W. K., \& Chapman, C. R. 1978, Icarus, 35,1

Gustafson, B. A. S. 1994, Annual Review of Earth and Planetary Sciences, 22, 553

Habing, H. J., et al. 1999, Nature, 401, 456

Habing, H. J., et al. 2001, A\&A, 365, 545 
Haisch, K. E. Jr., Lada, E. A., \& Lada, C. J. 2001, ApJ, 443, L153

Hayashi, C. 1981, Prog. Theor. Phys. Suppl., 70, 35

Hernández, J., et al. 2007, ApJ, 662, 1067

Hillenbrand, L. A., et al. 2008, ApJ, 677, 630

Hines, D. C., et al. 2007, ApJ, 671, 165

Holland, W. S., et al. 1998, Nature, 392, 788

Hollenbach, D., et al. 2005, ApJ, 631, 1180

Jura, M. 2004, ApJ, 603, 729

Kalas, P., Liu, M. C., \& Matthews, B. C. 2004, Science, 303, 1990

Kenyon, S. J., \& Bromley, B. C. 2001, AJ, 121, 538

Kenyon, S. J., \& Bromley, B. C. 2002, ApJ, 577, L35

Kenyon, S. J., \& Bromley, B. C. 2004, ApJ, 602, L133

Kenyon, S. J., \& Bromley, B. C. 2005, AJ, 130, 269

Kim, J. S., et al. 2005, ApJ, 632, 659

Kitamura, Y., Momose, M., Yokogawa, S., Kawabe, R., Tamura, M., \& Ida, S. 2002, ApJ, 581, 357

Koerner, D. W., Ressler, M. E., Werner, M. W., \& Backman, D. E. 1998, ApJ, 503, L83

Krivov, A. V., Queck, M., Löhne, T., \& Sremčević, M. 2007, A\&A, 462, 199

Lada, C. J., \& Lada, E. A. 2003, ARA\&A, 41, 57

Lada, C. J., et al. 2006, AJ, 131, 1574

Lagrange, A.-M., Backman, D. E., \& Artymowicz, P. 2000, in Protostars and Planets IV, ed. V. Mannings, A. P. Boss, \& S. S. Russell (Tucson, AZ: Univ. Arizona Press), 639

Lavalley, M., Isobe, T., \& Feigelson, E. 1992, in ASP Conf. Ser. 25, Astronomical Data Analysis Software and Systems I, ed. D. M. Worrall, C. Biemesderfer, \& J. Barnes (San Francisco, CA: ASP), 245

Lay, O. P., Carlstrom, J. E., \& Hills, R. E. 1997, ApJ, 489, 917

Liou, J.-C., \& Zook, H. A. 1999, AJ, 118, 580

Lissauer, J. J. 1987, Icarus, 69, 249

Mamajek, E., \& Hillenbrand, L. A. 2008, ApJ, 687, 1264

Mamajek, E. E., Meyer, M. R., Hinz, P. M., \& Hoffman, W. F. 2004, ApJ, 612, 496

Mannings, V., \& Barlow, M. J. 1998, ApJ, 497, 330

Mathis, J. S. 1990, ARA\&A, 28, 37

Meyer, M. R., Backman, D. E., Weinberger, A. J., \& Wyatt, M. C. 2007, in Protostars and Planets V, ed. B. Reipurth, D. Jewitt, \& K. Keil (Tucson, AZ: Univ. Arizona Press)

Meyer, M. R., Calvet, N., \& Hillenbrand, L. A. 1997, AJ, 114, 288

Meyer, M. R., et al. 2004, ApJS, 154, 422

Meyer, M. R., et al. 2006, PASP, 118, 1690

Meyer, M. R., et al. 2008, ApJ, 673, L181

Moór, A., Ábrahám, P., Derekas, A., Kiss, Cs., Kiss, L. L., Apai, D., Grady, C., \& Henning, Th. 2006, ApJ, 644, 525

Moro-Martín, A., \& Malhotra, R. 2003, AJ, 125, 2255

Moro-Martín, A., \& Malhotra, R. 2005, ApJ, 633, 1150

Moro-Martín, A., et al. 2007a, ApJ, 658, 1312

Moro-Martín, A., et al. 2007b, ApJ, 668, 1165

Padgett, D. L., et al. 2006, ApJ, 645, 1283

Papovich, C., et al. 2004, ApJS, 154, 70

Pascucci, I., et al. 2006, ApJ, 651, 1177
Pascucci, I., et al. 2007, ApJ, 663, 383

Plets, H., \& Vynckier, C. 1999, A\&A, 343, 496

Preibisch, T., \& Mamajek, E. , in Handbook of Low Mass Star Forming Regions, The Southern Sky, Vol. II, ed. B. Reipurth (San Francisco, CA: ASP), in press

Press, W. H., Teukolsky, S. A., Vetterling, W. T., \& Flannery, B. P. 2002, Numerical Recipes in $\mathrm{C}++$, (Cambridge: Cambridge Univ. Press)

Protassov, R., van Dyk, D. A., Connors, A., Kashyap, V. L., \& Siemiginowska, A. 2002, ApJ, 571, 545

Reach, W. T., et al. 1996, A\&A, 315, L381

Rebolo, R., Martiín, E. L., \& Magazzù, A. 1992, ApJ, 389, L83

Rhee, J. H., Song, I., \& Zuckerman, B. 2008, ApJ, 675, 777

Rhee, J. H., Song, I., Zuckerman, B., \& McElwain, M. 2007, ApJ, 660, 1556

Rieke, G. H. 2005, ApJ, 620, 1010

Safronov, V. S. 1969, in Evolyutsiya doplanetnogo oblaka i obrazovanie Zemli i planet, (Moscow: Nauka), English transl. Evolution of the Protoplanetary Cloud and Formation of the Earth and the Planets, NASA Tech. Transl. F-677, (Jerusalem: Israel Program Sci. Transl.) (1972))

Schneider, G., et al. 2006, ApJ, 650, 414

Siegler, N., Muzerolle, J., Young, E. T., Rieke, G. H., Mamajek, E. E., Trilling, D. E., Gorlova, N., \& Su, K. Y. L. 2007, ApJ, 654, 580

Silverstone, M. D., et al. 2006, ApJ, 639, 1138

Smith, B. A., \& Terrile, R. J. 1984, Science, 226, 1421

Song, I., Zuckerman, B., Weinberger, A. J., \& Becklin, E. E. 2005, Nature, 436, 363

Spangler, C., Sargent, A. I., Silverstone, M. D., Becklin, E. E., \& Zuckerman, B. 2001, ApJ, 555, 932

Spaute, D., Weidenschilling, S. J., Davis, D. R., \& Marzari, F. 1991, Icarus, 92, 147

Stauffer, J. R., et al. 2005, AJ, 130, 1834

Stern, S. A. 1996, A\&A, 310, 999

Su, K. Y. L., et al. 2006, ApJ, 653, 675

Takeuchi, T., \& Artymowicz, P. 2001, ApJ, 557, 990

Thébault, P., \& Augereau, J.-C. 2007, A\&A, 472, 169

Trilling, D. E., et al. 2008, ApJ, 675, 1086

Weidenschilling, S. J. 1977, Ap\&SS, 51, 153

Weingartner, J. C., \& Draine, B. T. 2001, ApJ, 548, 296

Werner, M., et al. 2004, ApJS, 154, 1

Wetherill, G. W., \& Stewart, G. R. 1989, Icarus, 77, 300

Williams, J. P., \& Andrews, S. M. 2006, ApJ, 653, 1480

Williams, D. R., \& Wetherill, G. W. 1994, Icarus, 107, 117

Wolf, S., \& Hillenbrand, L. A. 2003, ApJ, 496, 603

Wood, B. E., Müller, H.-R., Zank, G. P., \& Linsky, J. L. 2002, ApJ, 574, 412

Wood, B. E., Müller, H.-R., Zank, G. P., Linsky, J. L., \& Redfield, S. 2005, ApJ, $628, \mathrm{~L} 143$

Wyatt, M. C. 2003, ApJ, 598, 1321

Wyatt, M. C. 2005, A\&A, 433, 1007

Wyatt, M. C., Smith, R., Greaves, J. S., Beichman, C. A., Bryden, G., \& Lisse, C. M. 2007a, ApJ, 658, 569

Wyatt, M. C., Smith, R., Su, K. Y. L., Rieke, G. H., Greaves, J. S., Beichman, C. A., \& Bryden, G. 2007b, ApJ, 663, 365

Zuckerman, B., \& Song, I. 2004, ApJ, 603, 738 